University of Tennessee Health Science Center

UTHSC Digital Commons

\title{
Development and Implementation of Computer-Based Learning Modules on Alternative Communication for Pediatric Nurses and Nursing Students
}

Amanda Kaye Simmons

University of Tennessee Health Science Center

Follow this and additional works at: https://dc.uthsc.edu/dissertations

Part of the Family Practice Nursing Commons, Interprofessional Education Commons, Other Nursing Commons, Pediatrics Commons, Speech and Hearing Science Commons, and the Speech Pathology and Audiology Commons

\section{Recommended Citation}

Simmons, Amanda Kaye (https://orcid.org/0000-0002-4572-3101), "Development and Implementation of Computer-Based Learning Modules on Alternative Communication for Pediatric Nurses and Nursing Students" (2021). Theses and Dissertations (ETD). Paper 547. http://dx.doi.org/10.21007/ etd.cghs.2021.0532.

This Dissertation is brought to you for free and open access by the College of Graduate Health Sciences at UTHSC Digital Commons. It has been accepted for inclusion in Theses and Dissertations (ETD) by an authorized administrator of UTHSC Digital Commons. For more information, please contact jwelch30@uthsc.edu. 


\title{
Development and Implementation of Computer-Based Learning Modules on Alternative Communication for Pediatric Nurses and Nursing Students
}

\author{
Abstract \\ Purpose and Rationale. To develop, implement, and assess computer-based learning modules (CBLs) on \\ augmentative and alternative communication (AAC) with pediatric nurses and nursing students for \\ knowledge outcomes, self-efficacy, and perceived relevance and effectiveness. Effective communication \\ ensures all parties can understand and express themselves clearly, through any means necessary, often \\ including AAC tools, supports, and strategies. Communication is essential in healthcare but there is \\ limited research with pediatric nurses. Previous research with a single hospital indicated a need for nurse \\ education on AAC. This research used focus groups and interviews to understand pediatric nurse and \\ nursing student knowledge about AAC. The qualitative data was analyzed and used to create short CBLs \\ on AAC which were piloted and implemented with both groups. \\ Study Population. Fifteen pediatric nurses and nursing students participated in qualitative data collection, \\ five pediatric nurses and nursing students in the feasibility pilot of the CBLs, and 69 participants in the \\ CBL implementation phase. Participants were 18 years of age or older and a pediatric nurse or nursing \\ student.
}

Research Design and Study Procedures. Following Institutional Review Board (IRB) approval, qualitative data collection included focus groups and interviews with pediatric nurses and nursing students to gain feedback on participant experience with AAC. Data was gathered, transcribed using word-for-word transcription, and analyzed using grounded theory and thematic analysis to prioritize information for inclusion in CBLs for further implementation. CBLs were created using the analyzed qualitative data, piloted with five participants, and implemented with 69 participants using a pre-test, post-test design for three short online modules with both groups.

Results. Data analysis included thematic analysis of qualitative data using grounded theory. Statistical analyses included repeated measures analysis of variance (ANOVA), independent samples t-tests, multivariate analysis of variance (MANOVA), and descriptive statistics. Participants learned new information from pre- to post-test, increased their perceived self-efficacy to use the information provided, and overall felt it was relevant to their clinical practice and an effective method of providing information.

Document Type

Dissertation

Degree Name

Doctor of Philosophy (PhD)

Program

Speech and Hearing Science

Research Advisor

Jillian H. McCarthy, Ph.D.

\section{Keywords}

Augmentative and alternative communication; Computer-based learning modules; interdisciplinary; collaboration; nursing education 


\section{Subject Categories}

Communication Sciences and Disorders | Family Practice Nursing | Interprofessional Education | Medical Education | Medical Specialties | Medicine and Health Sciences | Nursing | Other Nursing | Pediatrics | Speech and Hearing Science | Speech Pathology and Audiology 
University of Tennessee HeAlth Science Center

DOCTORAL DisSERTATION

\section{Development and Implementation of Computer-Based Learning Modules on Alternative Communication for Pediatric Nurses and Nursing Students}

Author:

Amanda Kaye Simmons
Advisor:

Jillian McCarthy, Ph.D.

A Dissertation Presented for The Graduate Studies Council of

The University of Tennessee Health Science Center

in Partial Fulfillment of Requirements for the Doctor of Philosophy degree from

The University of Tennessee

in

Speech and Hearing Science: Speech Language Pathology

College of Graduate Health Sciences 
Copyright (C) 2021 by Amanda Kaye Simmons.

All rights reserved.

Modified with permission Masters/Doctoral Thesis LaTeX Template

Version $2.5(8 / 27 / 2017)$

http://www.LaTeXTemplates. com Creative Commons License CC BY-NC-SA 3.0 
This work is dedicated to the practicing and aspiring healthcare providers who support and provide a voice to children and families in some of their hardest days. 


\section{Acknowledgements}

This work would not have been possible without the love and support of my family, friends, co-workers, colleagues, and mentors. This list could never be exhaustive, but I hope to highlight key individuals who have been instrumental in the completion of this dissertation.

First, I would like to thank my mentor, Dr. Jillian McCarthy, who always listened and entertained my ideas and questions with enthusiasm and grace. She was essential in helping me strive for excellence while seeking to find that elusive work-life balance. She guided me through conducting strong research while allowing me the flexibility to conduct research that was meaningful and clinically relevant.

To my committee members, Drs. Hamby, Hedrick, Koszalinski, and Reilly, for their guidance and advice on my research, writing, and so much more. They were a well-balanced team who supported me through research set-backs, teaching woes, and life changes. I am a more well-rounded and capable individual for their time, attention, and input throughout my doctoral work.

To the University of Tennessee Health Science Center, Office of Equity and Diversity for supporting my research through a 2020 Diversity and Inclusion Mini-Grant. This funding support made it possible to put communication supports in the hands of nurses and students across the US.

To the Patient Provider Communication Network and Boston Children's Hospital, for providing freely available low-technology AAC resources to clinicians, families, and patients. For allowing me to share their work and tools with nurses and nursing student participants in my research; their contributions continue to benefit so many.

To my husband, Nathan Simmons, who not only provided hours of video editing for my education modules but has put up with my over-planning, frustration, fear, and selfdoubts. He was always ready with words of encouragement, a calming hug, or a needed distraction, knowing it would help me rest and come back into things newly invigorated. He is my best friend and the love of my life and I cannot wait for the next adventure together.

To my parents and sister, Paul and Michele McRoy and Kristin Baker, for always being my biggest fans whether at the opera, overseas, or in my research and clinical work. They are the first to arrive and the last to leave, no matter what it is. They are the family that I was given but that I would choose again and again. 
To my friend and co-researcher, Alaina Eck who was serendipitously assigned as my office mate and is now one of my dearest friends. She helped with qualitative data analysis and coding and so much more. She is always just a phone call away no matter the need research support, someone to verbally process with, or to provide emotional support.

Finally, to all of the nurses and nursing students who participated in my research over the years and provided feedback, advice, and words of encouragement. They are the reason I still have joy in this work after six years. Their dedication and hard work that I have gotten to witness first hand, in clinical work and research, gives me strength to continue fighting for the communication right of our patients and families. 


\section{Abstract}

Amanda Kaye Simmons

Development and Implementation of Computer-Based Learning

Modules on Alternative Communication for Pediatric Nurses and

Nursing Students

Purpose and Rationale. To develop, implement, and assess computer-based learning modules (CBLs) on augmentative and alternative communication (AAC) with pediatric nurses and nursing students for knowledge outcomes, self-efficacy, and perceived relevance and effectiveness. Effective communication ensures all parties can understand and express themselves clearly, through any means necessary, often including AAC tools, supports, and strategies. Communication is essential in healthcare but there is limited research with pediatric nurses. Previous research with a single hospital indicated a need for nurse education on AAC. This research used focus groups and interviews to understand pediatric nurse and nursing student knowledge about AAC. The qualitative data was analyzed and used to create short CBLs on AAC which were piloted and implemented with both groups.

Study Population. Fifteen pediatric nurses and nursing students participated in qualitative data collection, five pediatric nurses and nursing students in the feasibility pilot of the CBLs, and 69 participants in the CBL implementation phase. Participants were 18 years of age or older and a pediatric nurse or nursing student.

Research Design and Study Procedures. Following Institutional Review Board (IRB) approval, qualitative data collection included focus groups and interviews with pediatric nurses and nursing students to gain feedback on participant experience with AAC. Data was gathered, transcribed using word-for-word transcription, and analyzed using grounded theory and thematic analysis to prioritize information for inclusion in CBLs for further implementation. CBLs were created using the analyzed qualitative data, piloted with five participants, and implemented with 69 participants using a pre-test, post-test design for three short online modules with both groups.

Results. Data analysis included thematic analysis of qualitative data using grounded theory. Statistical analyses included repeated measures analysis of variance (ANOVA), independent samples t-tests, multivariate analysis of variance (MANOVA), and descriptive statistics. Participants learned new information from pre- to post-test, increased their 
perceived self-efficacy to use the information provided, and overall felt it was relevant to their clinical practice and an effective method of providing information. 


\section{Contents}

1 Introduction $\quad 1$

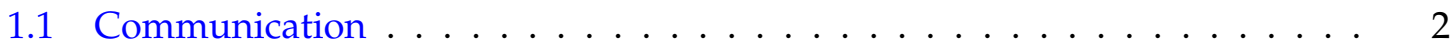

1.1.1 Patient-Provider Communication: Role and Importance . . . . . . . 3

1.1.2 Patients with Complex Communication Needs . . . . . . . . . . . . . 4

1.1.3 Communication Needs of Pediatric Patients in Medical Settings . . . 5

1.2 Previous Research . . . . . . . . . . . . . . . . . . . . 7

1.3 Theory and Research Inquiries f . . . . . . . . . . . . . 8

1.3.1 Theories ......................... 8

1.3.2 Research Inquiries . . . . . . . . . . . . . . . . . 9

2 Literature Review $\quad 11$

2.1 Communication and AAC . . . . . . . . . . . . . . 11

2.1 .1 AAC and $\mathrm{CCN} \ldots \ldots \ldots \ldots \ldots \ldots$

2.1 .2 Nurse Use of AAC . . . . . . . . . . . . . . . . . . . . . . . 14

2.1.3 Pediatric Nurse Use of AAC . . . . . . . . . . . . . . . . . . . . . 16

Importance of AAC for nurse-pediatric patient communication . . . 16

Barriers to using AAC for nurse-pediatric patient communication . . 17

Improving AAC use in the pediatric acute care setting $\ldots . . . .18$

Differences in AAC use with pediatric patients based upon unit . . . 19

2.1.4 Education with Nurses and Nursing Students . . . . . . . . . . . 19

Online education with nurses . . . . . . . . . . . . . 20

Online education with nursing students . . . . . . . . . . . . 20

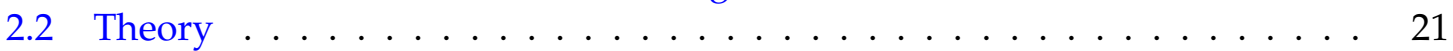

2.2.1 Participatory Action Research . . . . . . . . . . . . . . . . . . . 22

2.2.2 Grounded Theory and Thematic Analysis . . . . . . . . . . . . . . 22

2.2.3 Gagné's Cognitive Instructional Theory . . . . . . . . . . . . . . . 23

Processes of learning . . . . . . . . . . . . . . 23

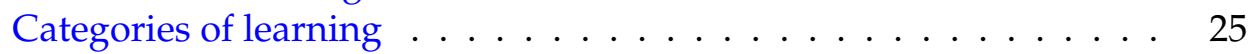

Conditions of learning . . . . . . . . . . . . . . . . 27

Events of instruction . . . . . . . . . . . . . . . . . . 28

2.2.4 Bandura's Social Cognitive Theory . . . . . . . . . . . . . . . . . . . . . . . . 29

Modeling and observational learning . . . . . . . . . . . . . . . . . 29

Self-efficacy . . . . . . . . . . . . . . . . 30 
3 Methodology 32

3.1 Focus Groups and Interviews $($ RI-1) . . . . . . . . . . . . . . . . . 32

3.2 Computer-Based Learning Modules (RI-2 and RI-3) . . . . . . . . . . . . . 34

3.2.1 Previously Developed CBLs . . . . . . . . . . . . . . . . . 34

3.2.2 Development and Preliminary Piloting of New CBLs . . . . . . . . 35

3.2.3 Trialing of New CBLs on AAC . . . . . . . . . . . . . 40

3.2 .4 Participants . . . . . . . . . . . . . . . . . 41

3.2 .5 Funding and Incentives . . . . . . . . . . . . . . . . . . . 43

4 Results 44

4.1 Qualitative Results . . . . . . . . . . . . . . . . . . . . 44

4.1.1 Pediatric Nurse Data - Interviews and Focus Groups . . . . . . . . . . 44

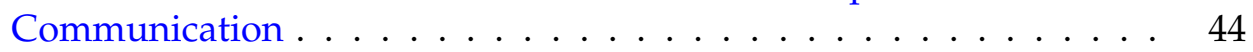

Clinical experience . . . . . . . . . . . . . . . . . . . . . . . . . . . . . . . 48

Education ........................... 50

4.1.2 Nursing Student Data - Interviews and Focus Groups . . . . . . . . . 52

Communication . . . . . . . . . . . . . . 52

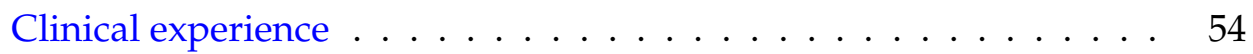

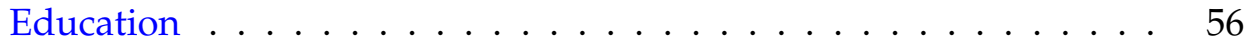

4.2 Quantitative Results . . . . . . . . . . . . . . . . . 57

4.2 .1 Knowledge . . . . . . . . . . . . . . . . . 57

4.2 .2 Self-Efficacy . . . . . . . . . . . . . . . . . 61

4.2 .3 Adapted L-SES . . . . . . . . . . . . . . . . . . . . . . 66

4.2 .4 Relevance of Content . . . . . . . . . . . . . . . . . . . . 67

4.2.5 Effectiveness of the Educator and Method . . . . . . . . . . . . 68

4.2 .6 Clinical Application . . . . . . . . . . . . . . . . 68

Module 1: Wait time and recasting . . . . . . . . . . . . 68

Module 2: Choice making and simple sign language $\ldots . . .70$

Module 3: Partner-assisted scanning . . . . . . . . . . . . 72

5 Discussion and Conclusions $\quad 77$

5.1 Qualitative Discussion . . . . . . . . . . . . . . . . . 77

5.1 .1 Pediatric Nurse Qualitative Data . . . . . . . . . . . . . . . . . . . 77

5.1 .2 Nursing Student Qualitative Data . . . . . . . . . . . . . . . . . . . . 79

5.2 Quantitative Discussion . . . . . . . . . . . . . . . . 81

5.3 Study Limitations . . . . . . . . . . . . . . . . . . . . . 86

5.4 Conclusions . . . . . . . . . . . . . . . . . . . 87

$\begin{array}{ll}\text { A Summary of Relevant Research } & 88\end{array}$

B Pre-Test and Post-Test Questions $\quad 90$

$\begin{array}{ll}\text { List of References } & 106\end{array}$ 


\section{List of Tables}

2.1 Use of Gagné's Events of Instruction in This Research. . . . . . . . . . . . 28

3.1 Pilot Pre- to Post-Test Scores. . . . . . . . . . . . . . . . . . . . . . . . . . 37

3.2 Pilot Post-Data Reliability. . . . . . . . . . . . . . . . . . . . 37

3.3 Pilot Post-Test Scale Averages. . . . . . . . . . . . . . . . . . . . . . . . 38

3.4 Demographic Information. . . . . . . . . . . . . . . . . . 42

4.1 Qualitative Themes and Definitions. . . . . . . . . . . . . . 45

4.2 Pre- and Post-Test Results. . . . . . . . . . . . . . . . . . . 58

4.3 Self-Efficacy Repeated Measures Results. . . . . . . . . . . . . . . . . . . . . . 62

4.4 Reliability Scores for Likert Scales. . . . . . . . . . . . . . . . . . . . . . . . . 65

4.5 Post-Test Scale Averages. . . . . . . . . . . . . . . . . . . . . . . . 67

4.6 Patients Who Would Benefit from Low-Technology AAC per Week. . . . . . 75 


\section{List of Figures}

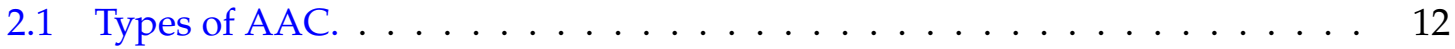

4.1 Module 1 Pre- to Post-Test Scores. . . . . . . . . . . . . . . . . . . . . . . . . . . . . 59

4.2 Module 2 Pre- to Post-Test Scores. . . . . . . . . . . . . . . . . . . . . . . 60

4.3 Module 3 Pre- to Post-Test Scores. . . . . . . . . . . . . . . . . . . . . . . . . . 61

4.4 Module 1 Self-Efficacy Scale Scores. . . . . . . . . . . . . . . . . . . . . . . . . . . . . . . . . . . 63

4.5 Module 2 Self-Efficacy Scale Scores. . . . . . . . . . . . . . . . . . . . . . . . . . . . . . . . . . . 64

4.6 Module 3 Self-Efficacy Scale Scores. . . . . . . . . . . . . . . . . . . . . . . . . . 64

4.7 Low-Technology Board Created by Participant. . . . . . . . . . . . . . . . . . . . 72

4.8 Top 5 Vocabulary for a Low-Technology Board. . . . . . . . . . . . . . . . . 74 


\section{List of Abbreviations}

$\begin{array}{ll}\text { AAC } & \text { Augmentative and Alternative Communication } \\ \text { ANOVA } & \text { Analysis of Variance } \\ \text { ASD } & \text { Autism Spectrum Disorder } \\ \text { AT } & \text { Assistive Technology } \\ \text { CBL } & \text { Computer-Based Learning Module } \\ \text { CCN } & \text { Complex Communication Needs } \\ \text { CEU } & \text { Continuing Education Units } \\ \text { CNA } & \text { Certified Nursing Assistant } \\ \text { CP } & \text { Cerebral Palsy } \\ \text { CVICU } & \text { Cardiovascular Intensive Care Unit } \\ \text { DS } & \text { Down Syndrome } \\ \text { ED } & \text { Emergency Department } \\ \text { EFA } & \text { Exploratory Factor Analysis } \\ \text { GLM } & \text { Generalized Linear Model } \\ \text { ICU } & \text { Intensive Care Unit } \\ \text { IRB } & \text { Institutional Review Board } \\ \text { LPN } & \text { License Practical Nurse } \\ \text { MANOVA } & \text { Multivariate Analysis of Variance } \\ \text { Med-Surg } & \text { Medical Surgical Oncology } \\ \text { NICU } & \text { Neonatal Intensive Care Unit } \\ \text { NJC } & \text { National Joint Committee for the Communication } \\ \text { ODL } & \text { Needs of Persons with Severe Disabilities } \\ \text { OWL } & \text { Organizational Development and Leadership } \\ \text { PAR } & \text { Observe, Wait, Listen } \\ \text { PAS } & \text { Participatory Action Research } \\ \text { PI } & \text { Partner-Assisted Scanning } \\ \text { PICU } & \text { Primary Investigator } \\ \text { PRN } & \text { Pediatric Intensive Care Unit } \\ \text { RI } & \text { pro re nata } \\ \text { RN } & \text { Research Inquiry } \\ \text { SLP } & \text { Registered Nurse } \\ \text { T\&A } & \text { Speech Language Pathologist } \\ \text { TBI } & \text { Tonsillectomy and Adenoidectomy } \\ & \text { Traumatic Brain Injury } \\ & \end{array}$


UT-AACL

UTK-CON

VoIP
University of Tennessee Augmentative and Alternative Communication Lab University of Tennessee - College of Nursing Voice over Internet Protocol 


\section{Chapter 1}

\section{Introduction}

Communication, the two-way exchange of information between two parties, is a basic human right (Rimal and Lapinski, 2009). The Communication Bill of Rights and the Human Rights Act (Brady et al., 2016; Equility and Human Rights Commission, 2014; United Nations, 1948) support that all individuals, regardless of type or severity of disability or impairment, should be able to control and affect their environment through communication. In addition, the National Joint Committee for the Communication Needs of Persons with Severe Disabilities (NJC) has provided guidelines for how to meet communication needs of even those individuals with the most severe complex communication needs (CCN; Brady et al., 2016).

We typically think of communication happening through verbal speech; however, individuals with disabilities, either temporary or permanent, may be unable to speak. In these instances, additional communication supports, such as augmentative and alternative communication (AAC) may be indicated. These supports can provide communication assistance to ensure clarity when providing a message or ensuring that a message is understood. Regardless of the method of communication or supports required, the ability to communicate via any means necessary impacts an individuals' autonomy and quality of life (Equility and Human Rights Commission, 2014; McLeod, 2018; United Nations, 1948).

The importance of effective communication is paramount in medical settings. Patients experience $\mathrm{CCN}$, whether temporary or permanent, at an increased incidence and prevalence during an acute medical event or hospitalization (Bartlett et al., 2008; The Joint Commission, 2020; Costello, L. Patak, and Pritchard, 2010; Divi et al., 2007). Hospitalized patients are at an increased risk for CCN and with higher stakes if they are unable to provide often required medical decisions. Patients with $\mathrm{CCN}$ are at an increased risk for sentinel events, defined as death or serious injury, during hospitalization. Additional ramifications include increased length of stay, increased cost to the healthcare system, and decreased

quality of life (Happ, Garrett, et al., 2014; Karlsen, Ølnes, and Heyn, 2019). The inverse has been found to be true when effective patient-provider communication is enlisted, with findings indicating increased positive patient outcomes, adherence to treatment regimens, increased patient safety, and improved satisfaction with care provided (Ong et al., 1995; J. K. Rao et al., 2007; Stewart, 1995). 
Communication in the healthcare setting involves a two-way exchange between healthcare providers and patients. All healthcare providers are charged with ensuring that an effective exchange of information is successful. This has been evidenced by mandates from the Joint Commission on Accreditation of Healthcare Organizations (JACHO) and the World Health Organization (WHO) that require all patients are provided with the means to communicate, through verbal, non-verbal, or alternative communication methods during their hospitalization (The Joint Commission, 2011; World Health Organisation, 2011).

Nurses serve as primary communication partners for patients and their families in medical settings (Pangh et al., 2019; D. M. Wolf et al., 2008). Thus, nurses should be versed in basic communication strategies and rudimentary AAC tools and techniques; however, in previous research, nurses have reported limited knowledge related to AAC and communication strategies outside of therapeutic communication (Simmons et al., 2019). Therapeutic communication, often discussed in-depth in nursing education, focuses on the development of rapport and an atmosphere of safety for patients and families during their medical encounter. This is an important aspect of bedside manner; however, the provision of accurate information exchange during a hospital stay is essential to provide appropriate patient care. Communication must be more than therapeutic, it must be holistic in ensuring accurate and thorough information exchange as well.

The reality is that many healthcare providers, including nurses, do not have access to in-depth education or training regarding alternative communication supports, strategies, and materials. This research seeks to fill that gap in education availability by developing, piloting, and implementing short, online, computer-based learning modules (CBLs) on noand low-technology AAC supports.

A foundation of research has been laid regarding the experience and benefits of AAC training with nurses working with adults in acute care (e.g., Balandin et al., 2007; Happ, Garrett, et al., 2014; Koszalinski, Tappen, and Viggiano, 2015); however, limited research exists with pediatric nurses and nursing students (Costello, 2000; Costello, L. Patak, and Pritchard, 2010; Costello, Santiago, and S. W. Blackstone, 2015; Thunberg, Buchholz, and Nilsson, 2016; Thunberg, Törnhage, and Nilsson, 2016). This research will focus on the potential benefits including knowledge exchange and self-efficacy measures with pediatric nurses and nursing students. Qualitative data with pediatric nurses and nursing students was gathered through interview and focus group discussions which have been analyzed to inform the development of three online education modules.

\subsection{Communication}

Communication occurs so often in our everyday lives that we easily take its complexity and necessity for granted. Communication is frequently thought of as the simple exchange of a message between a sender and receiver. In theory, this is part of what happens in effective communication; however, it is a far too simple model. Effective communication needs a sender to send a message to a receiver, but we must also consider whether the receiver is 
able to understand the message, interact with that information, and return a subsequent message to the original sender. This exchange must include the ability of both parties to take on and execute each role successfully over multiple iterations. Communication can be impacted by the interaction location and context (ex., is there background noise or distractions, what kind of information is being communicated), culture (ex., what are cultural considerations that must be considered or adapted for), and relationship (ex., coworker vs. family member), to name a few.

In addition to these considerations, we must consider the various verbal and nonverbal ways that we communicate. Verbal communication consists of words and language but may be oral or non-oral (ex., sign language would be a non-oral verbal communication act). Similarly, non-verbal communication, which plays a large role in the 'feel' of a conversation, can include oral and non-oral methods (ex., oral non-verbal could be laughter, non-oral non-verbal may be a facial expression). Both verbal and non-verbal communication can impact whether someone is willing to share or withhold information. Non-verbal communication can include a look between spouses or a parent and child which may not include a transparent message such as a "no!", but can be interpreted for meaning by most onlookers. In a similar fashion, we use non-verbal communication such as head nods, facial expressions, and gestures to communicate information every day. These same tools and strategies that prove helpful across a loud crowded room, or when attempted to maintain pragmatic appropriateness, are also beneficial to patients and healthcare providers if verbal speech is inhibited.

In the current age, communication is occurring more frequently in synchronous and asynchronous interactions over the internet through email, social media, or video chat. Thus, other modes of communication such as text communication (e.g., text messages, emails, chat box in a teleconference), video recordings, or emojis are providing new modes of exchanging information. Many communication modes that are becoming commonplace such as speech to text or text to speech, were originally created with individuals with disabilities in mind. As these new tools become more available and understood, they are additional ways that we can assist our patients in effectively communicating.

We take part in a variety of verbal and non-verbal communication interactions each day; however, individuals with $\mathrm{CCN}$, particularly those receiving care in medical settings, are more at risk for breakdowns in communication exchanges at the outset. Healthcare professionals are expected to meet patients' medical needs, necessitating the occurrence of effective patient-provider communication.

\subsubsection{Patient-Provider Communication: Role and Importance}

Patient-provider communication requires the effective back and forth exchange of information between healthcare provider (e.g., all healthcare providers interacting with patients) and patient. The Joint Commission states: 
Effective communication is the successful joint establishment of meaning wherein patients and health care providers exchange information, enabling patients to participate actively in their care from admission through discharge, and ensuring that the responsibilities of both patients and providers are understood. To be truly effective, communication requires a two-way process (expressive and receptive) in which messages are negotiated until the information is correctly understood by both parties. Successful communication takes place only when providers understand and integrate the information gleaned from patients, and when patients comprehend accurate, timely, complete, and unambiguous messages from providers in a way that enables them to participate responsibly in their care. (The Joint Commission, 2010 p. 1)

This is a tall order for healthcare providers under even the best of circumstances. Often healthcare providers such as doctors, nurses, nursing aids, and physical therapists, are pressed to provide high quality care with limited time allotted for each patient interaction. These patient-provider communication opportunities are often high stakes and can be stressful for everyone involved. Yet, it remains essential that patients and providers successfully provide and understand information in the two-way information exchange to ensure quality care and patient safety (The Joint Commission, 2011).

Research has found that education and training on communication with doctors, nurses, and other healthcare providers results in improved patient experience and satisfaction, decreased anxiety and stress, and improved patient health outcomes (e.g., Bartlett et al., 2008; Divi et al., 2007; J. K. Rao et al., 2007; Stewart, 1995; Zabar et al., 2006). In addition to improved patient experience and outcomes, the healthcare professionals who received education and training felt they were able to more effectively do their job as they could understand and treat patients more appropriately, following intentional communication exchanges (Pangh et al., 2019; Stewart, 1995; D. M. Wolf et al., 2008; Zabar et al., 2006).

\subsubsection{Patients with Complex Communication Needs}

Individuals with CCN (i.e., individuals unable to meet daily communication needs via spoken language because of an acquired or congenital disability; Therrien and Light, 2018) are estimated to make up 5-10\% of the general population and be present in up to $15 \%$ of hospital admissions (Bartlett et al., 2008). Individuals with CCN experience temporary or permanent communication deficits that can impact them in a variety of ways. A patient may be hospitalized following a car accident resulting in short-term intubation and mechanical ventilation, which will render them unable to speak verbally for a period of time. If this patient does not require a tracheotomy, he/she will likely be able to speak once he/she is able to breathe on their own following extubation. While this patient is unable to temporarily verbally communicate, other communication supports, such as AAC, will be needed.

Consider another patient hospitalized for a pulmonary infection who has cerebral palsy. This individual had limited verbal speech prior to their hospitalization and used 
a high-technology AAC device with eye-gaze and switch access to control their dynamic communication system. This individual is a long-term AAC user who will require communication support during their hospitalization, which may be new to their acute care healthcare providers. It should also be noted that these long-term AAC users often do not bring their primary AAC method (ex., high-tech AAC) to the hospital (e.g., Hemsley, Balandin, and Worrall, 2011) which can add an additional complication to an already complex hospital admission.

It is important for healthcare providers to work with patients and caregivers to determine what alternative communication supports they are familiar with and are most effective for this individual to ensure effective communication throughout their hospitalization, whether their primary communication system is available or not (e.g., Hemsley, Balandin, and Worrall, 2011). Individuals with CCN of any duration or severity require additional communication supports to ensure that their wishes are met, they are able to communicate wants and needs, and that they can engage in social interactions appropriately (Hopf, 2018; McLeod, 2018).

\subsubsection{Communication Needs of Pediatric Patients in Medical Settings}

Healthcare providers are charged with considering and managing communication for and with the pediatric patient and family unit holistically throughout their healthcare journey. It is possible to prepare for and at times prevent communication breakdowns as well as find ways to remedy them when they occur; however, this is done differently for adults than children.

Pediatrics, often touted as little adults, differ from 'grown-ups' in a variety of ways including physical and cognitive development. Kids have both developing bodies and motor systems as well as speech and language systems. Thus, children require different communication supports than adults which may include simplification and shortening of language, picture supports, and wait time. Children, as their language systems develop, oftentimes communicate using non-verbals and behaviors more than adults with fully developed language systems. Knowing the differences between adults and pediatrics, we must consider how we can adapt commonly used and researched communication strategies to be used with pediatrics in acute care settings. These considerations have been made outside of medical settings as children's communication is supported in the community and schools. These considerations often focus on the education and training of communication partners who must engage in this two-way communication exchange. In the pediatric acute care setting, nurses are a likely communication partner who might benefit from education and training on alternative communication strategies to support patient and family-centered care.

Patient and family centered care is the standard of care in pediatric healthcare settings as the family is recognized as the primary support system for most children. Additionally, it is important to include the patient and family in decisions related to patient care, as the 
patient and family are considered active care team members (Committee on Hospital Care. American Academy of Pediatrics, 2012).

Often, communication with families is hindered not by communication difficulties but by the situation and emotions revolving around the hospitalization or medical care required. These realities are important to keep in mind when interacting with families. Children, the actual pediatric patient, may be overlooked in communication exchanges because it is 'easier' to talk to the parents (Hemsley, Lee, et al., 2013; Simmons et al., 2019). However, it is important to remember that children also have communication rights which are specifically explained within the Convention on the Rights of the Child (UN Commission on Human Rights, 1990), particularly in Articles 2, 12, 13, 29, 30 and 40. Articles 12 and 13 relate to freedom of opinion and expression:

Article 12. 1. States Parties shall assure to the child who is capable of forming his or her own views the right to express those views freely in all matters affecting the child, the views of the child being given due weight in accordance with the age and maturity of the child. . .

Article 13. 1. The child shall have the right to freedom of expression; this right shall include freedom to seek, receive and impart information and ideas of all kinds, regardless of frontiers, either orally, in writing or in print, in the form of art, or through any other media of the child's choice. (UN Commission on Human Rights, 1990, p. 4)

Ensuring patient and family-centered care often requires the provision of communication supports. Communication breakdowns in pediatric medical settings result in malpractice suits because of poor understanding of patient or family perspectives and views, inadequate communication of information, and inaccessibility to providers (Committee on Hospital Care. American Academy of Pediatrics, 2012). Additional potential detriments of communication breakdowns include preventable adverse and sentinel events (The Joint Commission, 2020). Sentinel events are defined by the Joint Commission as, "a patient safety event that results in death, permanent harm, or severe temporary harm" (The Joint Commission, 2020). Research has found that many communication breakdowns are the result of the same communication breakdowns mentioned above that may lead to malpractice suits (Committee on Hospital Care. American Academy of Pediatrics, 2012). The Joint Commission, as mentioned previously, has provided standards and guidelines to assist acute care settings in thinking through ways to better support communication and prevent communication breakdowns which may lead to adverse medical events or worse (The Joint Commission, 2010). 


\subsection{Previous Research}

Limited research exists that assesses the education and training of pediatric nurses in acute care settings. Our research team has built upon this small foundation of knowledge through mixed methods research including surveys, interviews, and the trialing of education modules with nurses at a single pediatric hospital (Simmons et al., 2019).

Surveys and interviews were developed to determine the communication experiences and felt educational needs regarding AAC and communication for pediatric acute care nurses at a single hospital. Results were used to develop four, 30-minute CBLs on general AAC knowledge to meet these educational requests. These CBLs were created and trialed with four pediatric acute care nurses from a single hospital in East Tennessee. Positive knowledge outcomes were observed as well as perceived relevance of the content; however, participants reported that the CBLs were too long. Thus, this dissertation has built upon our previous research through focus group and interview discussions with pediatric acute care nurses and nursing students to develop new education modules. These modules were piloted to ensure clarity and validity, followed by an implementation trial to assess knowledge outcomes, perceived relevance and effectiveness of the CBL content, and improved self-efficacy using AAC with a larger sample of pediatric nurses and nursing students.

This research builds on a small foundation of educational research on alternative communication strategies for pediatric nurses. Costello (2000) developed and implemented an AAC program with demonstrated benefit to pediatric patients, families, and healthcare providers involved at a single hospital. Additional beneficial AAC education research has been conducted in narrow, often hospital-specific situations (e.g., Costello, L. Patak, and Pritchard, 2010; Thunberg, Buchholz, and Nilsson, 2016; Johnson, Nilsson, and Adolfsson, 2015.

The current research assists in the development of clinical education materials to support a broad network of nursing care in pediatric acute care settings and provides additional education to nursing students. JACHO and the $\mathrm{WHO}$ have mandated that hospitals provide and support communication efforts of patients (The Joint Commission, 2011; World Health Organisation, 2011) and research has demonstrated that the absence of effective communication leads to increased sentinel events (incidents of death or serious injury), decreased quality of life, prolonged hospitalization, and increased financial burden to hospital systems (The Joint Commission, 2020; Divi et al., 2007; Zaga, Berney, and Vogel, 2019). This research seeks to provide vital education to front line healthcare providers in an effort to bridge the knowledge and access gap reported and assist in ensuring effective communication efforts be made in pediatric acute care settings. 


\subsection{Theory and Research Inquiries}

\subsubsection{Theories}

Theories and theoretical frameworks guide research and help to support the design and study of a variety of measures. This research enlists multiple theoretical frameworks supporting the mixed methods design and data interpretation, beginning with the use of participatory action research (PAR) which subscribes to the idea that the researcher may not hold the group's perspective or experience and thus, it is beneficial to collect information from the individuals or population for whom you want to work with or study. Including them in the development and design of research is imperative as they may be impacted by the outcome of the inquiry (Ehde et al., 2013; White, Suchowierska, and M. Campbell, 2004). The initial phase of this research enlisted focus group discussions and individual semi-structured interviews with pediatric nurses and nursing students to learn about their experiences and preferences regarding communication education, experience, and needs.

This information was gathered in a way that allowed pediatric nurses and nursing students to communicate their experiences, preferences, and advice to the researcher. Their feedback and responses were analyzed using grounded theory and thematic analysis (see Chapter 2) to glean and enlist pertinent information in the development of education modules and pre- and post-test questions.

The qualitative data was analyzed using grounded theory and thematic coding to provide in-depth qualitative analysis yet ensure information was distilled to overarching themes that could be targeted in education modules. Grounded theory uses an iterative process of data collection and analysis leading to theory development which emerges from the gathered data (Glaser and Strauss, 1967). Thematic coding, a part of grounded theory, further clarifies the data through the development of themes that are applicable across more than a single participant or situation to the broader context of data (Flick, 2014). Thematic coding has been utilized in this research to form generalizations and identify information gaps to be addressed in the developed educational materials for pediatric acute care nurses and nursing students.

The development of the CBLs utilized the framework of Bandura's social cognitive theory which highlights the importance of self through self-observation, self-evaluation, self-reaction, and self-efficacy (Bandura, 1977a; Bandura, 1986). It also supports that social interactions, including observation of a skill or concept, can result in learning (Bandura, 1986; Bandura, 1998; Ziegler et al., 2005). This research has leaned heavily on the social cognitive theory, with a focus on participants' self-evaluation and self-efficacy measures. This theory informed the organization, inclusion of vignettes, and multi-sensory input (ex., auditory, orthographic, and visual) in the education modules. The CBLs included the use of taught information as well as video demonstrations.

Finally, Gagné's cognitive instructional theory was used in forming this educational 
research. Gagné's theoretical framework incorporates internal conditions (previous experience gathered through PAR in the form of interviews and focus groups) and external conditions (information being taught through these education modules) and outlines nine events of instruction. All aspects of this instructional theory have been considered and the nine events of instruction have been enlisted in the development of these education modules as discussed in Chapter 2.

\subsubsection{Research Inquiries}

The research inquiries (RI), considering the foundation of research we hope to build upon as well as the theoretical frameworks guiding our study are as follows, with our proposed hypotheses below:

RI - 1: What content regarding communication and AAC do pediatric nurses and nursing students find relevant to their clinical work as indicated during interview and focus group discussions?

We hypothesize that pediatric nurses and nursing students will find information related to AAC relevant to their clinical work, but that they will communicate limited knowledge or mastery. This hypothesis is supported by previous research by the PI (Simmons et al., 2019). This research will collect qualitative data through PAR to gain information and viewpoints from pediatric nurses and incorporate opinions and experiences and nursing students from across the United States.

RI - 2: Based on pre- and post-test results from the computer-based learning modules, how do pediatric nurses and nursing students'

a. Knowledge of AAC change?

It is hypothesized that pediatric nurses and nursing students will demonstrate knowledge gains from pre- to post-test for each of these education modules. This research has used relevant theories of education as well as assessment tools that have been piloted and validated with speech-language pathologists, pediatric nurses, and nursing students.

b. Self-efficacy regarding the use of AAC change through the use of a validated self-efficacy tool for clinical skills (Kang et al., 2019)?

It is hypothesized that pediatric nurses and nursing students will demonstrate an increase in self-efficacy from pre- to post-test as they learn new information and are given the opportunity to observe clinical scenarios with use of no- and low-tech AAC strategies in the CBLs. They will also be provided with low-tech AAC boards, if they choose, to use in their clinical work to assist in building their confidence and feelings of self-efficacy in putting this new knowledge into practice. 
RI - 3: Based on post-assessment results from the CBLs, how do pediatric nurses and nursing students rate:

a. Adapted Learning Self-Efficacy Scale (Cognitive, Affective, Psychomotor) It is hypothesized that pediatric nurses and nursing students will report cognitive, affective, and psychomotor self-efficacy scores greater than or equal to 4 on the Learning Self-Efficacy Scale (L-SES; Kang et al., 2019) indicating a strongly perceived benefit regarding cognitive, affective, and psychomotor abilities following this education.

b. Relevance of Content It is hypothesized that pediatric nurses and nursing students will report high content relevance for each module as evidenced by an average scale score of greater than or equal to 4 indicating participant perception of highly relevant content. This finding would demonstrate similar relevance data found in previous CBL research by the PI and the pilot data from this research.

c. Effectiveness of the Educator and Method It is hypothesized that pediatric nurses and nursing students will report effectiveness of the educator and CBLs with scores greater than or equal to 4 , indicating perceived highly effective information and method, in line with pilot data and previous research using CBLs by the PI. 


\section{Chapter 2}

\section{Literature Review}

\subsection{Communication and AAC}

Communication's role in our lives is often overlooked and undervalued; however, effective communication, a back and forth understanding of others and expression of ourselves, is required for everyday interactions. We can overlook the necessary role that communication plays until we are confronted with a difficult communication interaction such as conversing with someone who speaks a different language or the loss of our voice due to illness. When we find ourselves in a situation where the complexity of communication is highlighted, we get a glimpse into the reality of everyday life for those with CCN.

Effective communication, as defined in Chapter 1 by The Joint Commission, requires the "joint establishment of meaning" (The Joint Commission, 2010) between parties through a series of back and forth exchanges of information requiring both expression and understanding. Communication can be verbal (consisting of language) and non-verbal (communicating information without the use of language) and either can be oral or non-oral, as mentioned in Chapter 1. Research and clinical experience make clear the importance of effective communication in medical settings between healthcare providers and patients of all ages and diagnoses (Chapman, 2009; Feraco et al., 2016; The Joint Commission, 2010; L. Patak et al., 2009; P. R. Rao, 2011).

AAC is a type of Assistive Technology (AT) that seeks to support communication through augmenting an individual's communication abilities (e.g., using a communication board to clarify a message) or as an alternative system if he/she is unable to communicate verbally (e.g., the use of a high-technology device with eye-gaze and communication

software). AAC is defined by the International Society for Augmentative and Alternative Communication (ISAAC) as

a set of tools and strategies that an individual uses to solve everyday communicative challenges. Communication can take many forms such as: speech, a shared glance, text, gestures, facial expressions, touch, sign language, symbols, pictures, speech-generating devices, etc. Everyone uses multiple forms of communication, based upon the context and our communication partner. 


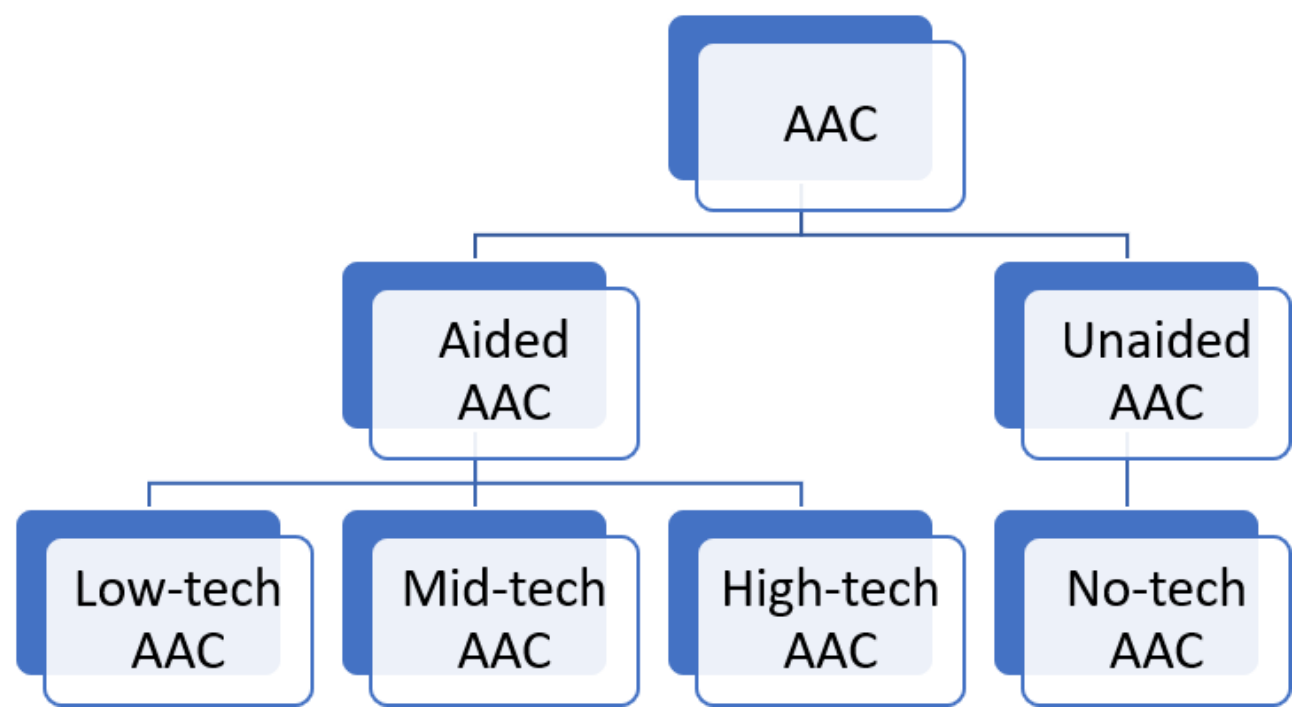

Figure 2.1: Types of AAC.

Effective communication occurs when the intent and meaning of one individual is understood by another person. The form is less important than the successful understanding of the message. (Burkhart, 2020, para. 1)

The primary purpose of AAC as mentioned in that definition is effective communication, not necessarily promoting a certain method or type of communication support. However, it can be helpful to think about available communication supports based on the level of technology required, thus, often used descriptions of AAC may include aided or unaided AAC, or no-technology, low-technology, mid-technology (light-tech), and hightechnology (All About AAC, 2021; Autism Adventures, 2021; Communication Community, 2021; Marx and Locast, n.d.; USSAAC, 2021; Moorcroft, Scarinci, and Meyer, 2019). These terms are helpful in considering the type of technical support which may be required and may, though not always, be helpful in considering types of AAC appropriate for different patients or clients.

Unaided AAC does not require anything outside of your body (e.g., sign language, facial expressions). No-technology AAC falls under the category of unaided AAC, requiring nothing outside of the communicator such as gestures. Aided AAC, by comparison, does require something outside of your body and includes low-technology, mid-technology, and high-technology supports. A graphic categorizing these types of AAC supports can be found in Figure 2.1.

No-technology AAC requires, as its name indicates, no technology and can be considered synonymous with unaided AAC. Examples of no-tech AAC include facial expressions, 
gestures, sign language, and vocalizations; any way information is communicated without the aid of something outside of your body.

Low-technology AAC is a type of aided AAC that includes the use of easily accessible items which require no power supply. Examples of low-technology AAC include paper or a white board and an appropriate writing utensil, a clear page protector which can be written on with a dry erase marker, a communication board with symbols, icons, letters, or pictures. These items are inexpensive to create and use; however, may be limiting in their functionality as they are static or unchanging systems. A picture board, for example, is limited in what can be communicated by the number of cells or pictures included. A letter board; however, is a static system which can be used to communicate a variety of words and messages. This highlights the importance of literacy and the consideration of literacy skills when determining the AAC tools that will reside in one's AAC tool box.

Mid-technology or light-tech AAC, though a less frequent AAC support and term, has a role to play and includes supports which require a power source (ex., batteries) and often use digitized or recorded speech if speech output is available. Examples of midtechnology AAC include switch-adapted toys and switches with recording capabilities such as the Ablenet's BIGMack ${ }^{T M}$ or little Step-by-Step ${ }^{T M}$.

High-technology AAC has become more common in the last ten years with the technology boom and the widespread availability of tablets, laptops, and smartphones. High-technology AAC is often more expensive than the other AAC options, includes options to use static and/or dynamic displays, requires a power source (e.g., battery or electrical outlet), and uses digitized (recorded) speech or synthesized (computerized) speech. These devices can be highly tailored to their users and can accommodate a variety of access methods including touch, switch, and eye gaze.

There is great variety in the AAC supports available to aid effective communication from the use of common items (e.g., pen and paper, page protector) to those available commercially. It is important to consider all options and to work to create a 'toolbox' of AAC supports for individuals with $\mathrm{CCN}$ as AAC is not a one-size fits all for individuals or situations (ex., a high-technology device or tablet should not go into the swimming pool or shower).

\subsubsection{AAC and CCN}

Many individuals struggle to communicate, temporarily or permanently, for a variety of reasons such as language barriers, language disorders, or medical complications. We cannot always prepare for changes in communication needs and thus the implementation of AAC may be required to replace lost abilities or supplement remaining communication strengths for a time. AAC is a part of the speech language pathologist's (SLPs) scope of practice and provides communication support for these diverse situations and patient populations. The Technology-Related Assistance to Individuals with Disabilities Act of 1988 (GovTrack.us, 2019) defines AAC as, "any item, piece of equipment, or product system 
whether acquired off the shelf, modified or customized, that is used to increase, maintain, or improve functional capabilities of individuals with disabilities."

SLPs can provide education and training for primary caregivers, teachers and support staff, and schools as well as healthcare staff in medical settings to ensure they have the knowledge and tools to provide communication supports across settings and situations. AAC implementation has been effective and beneficial to a variety of patient populations over the years (e.g., Light, McNaughton, et al., 2019; Light and K. Drager, 2007; Beukelman, Ball, and Fager, 2008; S. W. Blackstone, Beukelman, and K. M. Yorkston, 2015; Radtke, Tate, and Happ, 2012). A large body of literature exists to support the use of AAC with children with developmental disabilities such as cerebral palsy (CP), or autism spectrum disorder (ASD; e.g., Costello, Santiago, and S. W. Blackstone, 2015; Finke, Light, and Kitko, 2008; Finke, D. B. Mcnaughton, and K. D. R. Drager, 2009; Light and K. Drager, 2007; Light, Page, et al., 2007; Light and McNaughton, 2012; Light and D. Mcnaughton, 2015); however, more recently, AAC use in acute care medical settings has been considered and deemed beneficial (e.g., Costello, L. Patak, and Pritchard, 2010; Happ, Garrett, et al., 2014; Hemsley, Balandin, and Worrall, 2011; Koszalinski, Tappen, and Viggiano, 2015. Zaga, Berney, and Vogel (2019) completed a systematic literature review to consider the feasibility, utility, and safety of alternative communication interventions in ventilated ICU patients. They found that low-technology AAC such as communication boards and high-technology AAC devices such as tablets with communication applications or communication devices were the most effective based upon the current literature (Zaga, Berney, and Vogel, 2019).

A medical emergency and the subsequent recovery process are a common reason that someone may be temporarily or permanently unable to communicate effectively using verbal speech. There are few clinical researchers delving into the feasibility and reliability of using AAC in acute care medical settings to attempt to meet communication breakdowns with the adult and pediatric populations (e.g., Costello, L. Patak, and Pritchard, 2010; Happ, Baumann, et al., 2010; Hemsley, Balandin, and Togher, 2008b; Koszalinski, Tappen, and Viggiano, 2015; Pangh et al., 2019; Zaga, Berney, and Vogel, 2019). As this research focuses on the use of AAC education and supports with pediatric nurses and nursing students, this literature review will focus on the current state of the literature with regards to those populations.

\subsubsection{Nurse Use of AAC}

Nurses provide care for diverse patient populations with a variety of needs and potential communication difficulties. The current state of the research regarding AAC use by nurses with adult and pediatric populations has been concentrated in intensive care units (ICUs) with much of the research focusing on qualitative, descriptive, information-gathering, and initial pilot and implementation studies on AAC education, training, and interventions with different populations (ex., K. Dithole et al., 2016; K. S. Dithole et al., 2017; Happ, Garrett, et al., 2014; Hemsley, Balandin, and Togher, 2008b; Radtke, Tate, and Happ, 2012; Rodriguez, Spring, and Rowe, 2018). AAC is a diverse field incorporating communication 
strategies with tools, methods, and devices. To use AAC in an acute care setting requires a complex process which begins with education and training (Costello, 2000; Costello, L. Patak, and Pritchard, 2010; Happ, Garrett, et al., 2014; Holden, 2017; Koszalinski, Tappen, and Viggiano, 2015). The literature encourages the continued development of education and training programs for effective dissemination of AAC knowledge and experience in ICUs and beyond (Sarah W. Blackstone, 2007; S. W. Blackstone, Beukelman, and K. M. Yorkston, 2015; S. W. Blackstone and Pressman, 2016; El-Soussi et al., 2015; Finke, Light, and Kitko, 2008; Hemsley and Balandin, 2007; Hurtig et al., 2019; Lance Patak et al., 2006; L. Patak et al., 2009).

The available literature on qualitative and quantitative outcome measures for communication between nurses and adult patients has demonstrated improvement following training, feedback, and support (Happ, Sereika, et al., 2008; Happ, Baumann, et al., 2010; Happ, Garrett, et al., 2014; Radtke, Baumann, et al., 2011; Radtke, Tate, and Happ, 2012). Researchers have collected and analyzed qualitative data from patients, loved ones, caretakers, and healthcare professionals to determine communication priorities, barriers, and experiences (Balandin et al., 2007; Fatahi et al., 2010; Hemsley and Balandin, 2007; Hemsley, Balandin, and Togher, 2008a; Hemsley, Balandin, and Togher, 2008b; Hemsley, Balandin, and Worrall, 2011; Hemsley, Lee, et al., 2013; Noguchi, Inoue, and Yokota, 2019; Rodriguez, Spring, and Rowe, 2018; Wojnicki-Johansson, 2001; Zaga, Berney, and Vogel, 2019). Rodriguez, Spring, and Rowe, 2018 found, through focus group discussions, that when nurses commonly caring for patients who were 'suddenly speechless' were provided with training and access to a tablet-based communication device and software, they saw reduced frustration for patients and nurses, time saved, and efficient communication between patient and provider.

Much of the current literature focuses on the short-term use of AAC with patients who have temporary CCNs (Brunner et al., 2018; K. S. Dithole et al., 2017; El-Soussi et al., 2015; Hosseini, Valizad-Hasanloei, and Feizi, 2018; Johnson, Nilsson, and Adolfsson, 2015; Otuzoğlu and Karahan, 2014; Wojnicki-Johansson, 2001). The use of low-tech AAC (ex., communication boards) (Hosseini, Valizad-Hasanloei, and Feizi, 2018; Otuzoğlu and Karahan, 2014) as well as high-tech supports such as an iPad (Brunner et al., 2018) have been evidenced in the literature. One benefit of research with patients with temporary CCNs is the ability to, after the fact, ask them to reflect back on their experiences while unable to verbally communicate. Johnson, Nilsson, and Adolfsson, 2015 conducted interviews with nine patients who had undergone mechanical ventilation on a critical care unit, a number of whom mentioned their feelings of disconnection when unable to communicate clearly with their healthcare providers. The potential populations of individuals who might benefit from this type of AAC are vast, including anyone requiring emergency surgery, befalling an accident, or experiencing a change in medical status necessitating support such as intubation or mechanical ventilation. However, there is a significant population of individuals who use AAC daily for communication support, who often do not bring their AAC device to the hospital (Hemsley, Balandin, and Togher, 2008a). These populations require nurses to have a different knowledge base and set of skills which should be taken 
into consideration when developing AAC education and training (Morris, Dudgeon, and K. Yorkston, 2013; L. Patak et al., 2009).

Current research indicates that AAC methods and strategies are effective when enlisted promptly, by well-trained individuals confident in their abilities to use AAC, and with the support and buy-in of other healthcare staff, families, and patients in the acute care setting (Costello, 2000; El-Soussi et al., 2015; Happ, Garrett, et al., 2014; Lance Patak et al., 2006; Rodriguez, Spring, and Rowe, 2018). Due to the focus of this research, we will dig deeply into the literature existing on nurse use of AAC specifically with pediatric acute care patients and nursing student experience and use with AAC. Primary areas of consideration for the literature will include: (a) the importance of AAC use to facilitate nursepatient communication, (b) the barriers to using AAC for nurse-patient communication, (c) recommendations on how to improve AAC use in acute care, and (d) differences in AAC use based upon unit or educational experience. Each area of interest will be discussed below with an overview of the included articles found in Appendix A.1.

\subsubsection{Pediatric Nurse Use of AAC}

Communication with pediatric acute care patients calls for a specialized approach, including unique vocabulary and strategies. Many pediatric hospitals utilize family-centered care, which encourages high quality care focused on open communication and partnership between healthcare staff, family, and the patient (Committee on Hospital Care. American Academy of Pediatrics, 2012). This foundation of family-centered care promotes effective communication; however, during the acute care experience, there may be instances when new complex communication situations arise. In the event of a medical status change, surgery, or medical intervention, a child may be rendered unable to speak temporarily or permanently. There may be situations in which parents and family members are unable to understand their now non-verbal child, and AAC can assist in supporting those communication gaps.

There is a significant need for AAC at the pediatric bedside, as for adults (Markov and Hazan, 2012). There is limited research assessing the use and implementation of AAC in the pediatric acute care setting. Our current review of the literature yielded main sources from varied hospital units (pre- and post-operative intervention, day surgery, a general pediatric hospital, and the ICU). Data from multiple perspectives including that of the nurse or healthcare staff, patient, and parent or caregiver were found.

\section{Importance of AAC for nurse-pediatric patient communication}

The importance of AAC use with pediatric patients is undeniable, though there is limited research assessing how the implementation is best achieved. All studies in this review demonstrated significant benefit (quantitative and qualitative) from AAC implementation for the patient, nurse, and family members during the acute care process. Mesko et al. (2011) facilitated pediatric patients' communication of pain and its location with the use of AAC 
supports. They found that the location children identified was not always where nurses assumed their pain would be (e.g., nurses assumed patients' pain would be tied to a recent procedure but sometimes patients wanted to indicate pain elsewhere such as a headache; Mesko et al., 2011), which may impact the care received. Parents reported significant comfort and appreciation as they watched their children use AAC to communicate what was hurting and receive subsequent specific care (Mesko et al., 2011).

Throughout the literature, parents had strong opinions regarding the importance of communication and AAC for their child during hospitalization (Costello, 2000; Thunberg, Buchholz, and Nilsson, 2016). Whether they were the parent of a child with a temporary or permanent $\mathrm{CCN}$, all were adamant that nurses have knowledge on how best to communicate with their child and that they take the time to implement AAC and communication strategies (Costello, 2000; Thunberg, Buchholz, and Nilsson, 2016). Parents reported a significant benefit when they received AAC training before their child's surgery (Costello, 2000), stating that this preparation allowed the patient and family to become familiar with the communication method or device, practice using it, and at times voice bank (collect recordings of their child's voice to use on a communication device at a later time) in preparation for the upcoming surgery (Costello, 2000).

Costello, L. Patak, and Pritchard (2010) discussed AAC implementation practices informed by a patient's medical status that were used in the pediatric ICU (PICU). They outlined the importance of paying attention to the patient's alertness and awareness when deciding what AAC method or device to use (Costello, L. Patak, and Pritchard, 2010). Their recommendations for possible AAC implementation between nurses and patients at their facility were broken down into three possible phases: emerging from sedation, increased wakefulness, and need for broad and diverse communication access (Costello, L. Patak, and Pritchard, 2010).

Thunberg, Buchholz, and Nilsson (2016) gathered feedback from parents of children with $\mathrm{CCN}$ regarding hospitalization experiences in which they were able to communicate their desire for individualized care for their children, including a desire for nurse continuity and advanced AAC education and training for nurses. Thunberg, Törnhage, and Nilsson (2016) used saliva cortisol measures to gauge anxiety to see if using AAC schedules during planned surgery for kids with CCN might reduce their agitation. They found that the AAC schedule successfully decreased the need for additional medication before surgery and decreased the participant's post-surgery anxiety as indicated by saliva cortisol measures (Thunberg, Buchholz, and Nilsson, 2016). AAC may benefit kids with CCN, temporarily or permanently, during hospitalization.

\section{Barriers to using AAC for nurse-pediatric patient communication}

Barriers discussed in the research literature included the need for additional nurse knowledge and experience using AAC. In addition, attitudes towards the use of AAC play a large role when considering whether strategies and tools will be attempted with patients. Hemsley, Lee, et al. (2013) found that nurses had little knowledge regarding AAC but also 
cited time constraints, lack of available storage, difficulty mounting devices, and preference to communicate with parents at the bedside as reasons that AAC was not used. Hemsley, Lee, et al. (2013) conducted a qualitative research study to determine the experiences and opinions of healthcare staff and nurse's interactions with pediatric patients with cerebral palsy (CP). They found a significant attitude or knowledge barrier as, according to nurses, AAC was not necessary for kids with $\mathrm{CP}$, due to the perception that they do not have a need to communicate more than their immediate wants and needs, which could be anticipated (Hemsley, Lee, et al., 2013).

Additionally, physical barriers to using AAC in a patient's room were cited, such as difficulty setting up a device. Nurses reported that patients had difficulty accessing communication devices during hospitalization possibly due to motor impairments, the cumbersome nature of medical equipment, or device access difficulties (Hemsley, Lee, et al., 2013). Some nurses stated that AAC was difficult to use at the bedside or that it required too much space in already crowded patient rooms (Costello, 2000; Hemsley, Lee, et al., 2013; Hemsley and Balandin, 2014).

\section{Improving AAC use in the pediatric acute care setting}

The recommendations from parents and nurses focused on a need for more exposure to AAC through training and use on medical units. Parents of children with $\mathrm{CP}$ and $\mathrm{CCN}$ recommended that nurses receive additional training in communication and AAC (Thunberg, Buchholz, and Nilsson, 2016). Additional recommendations included the repetition of intervention and feasibility studies for AAC implementation. More research is needed to solidify the role AAC supports play such as a pain locator and intensity tool or a photographic schedule for day-surgery pediatric patients (Mesko et al., 2011; Thunberg, Buchholz, and Nilsson, 2016).

More research exists on the use of AAC by nurses with adult patients than pediatrics. There is a need for additional research with pediatric patients to ensure they are receiving the highest quality care during hospitalization. Pediatric patients are not just little adults, and therefore, education, training, and AAC strategies specific to the needs of these patients must be developed.

Numerous studies have been conducted in the last five years, indicating an increased awareness and curiosity regarding AAC training and use. This promising trajectory should continue as efficient training methods are created and trialed for nurses, healthcare staff, patients, and families. There is an additional need for improving AAC methods and devices for use with a variety of patient populations. Recommendations and feedback from patients, families, and healthcare staff should be taken into consideration to ensure that AAC devices are portable, easy to use, easy to clean, adaptable, and functionally relevant to users' needs.

Continued research exploring the perspectives and experiences of patients and healthcare staff is warranted. Next steps include the development and trialing of training programs and new interventions to prepare healthcare staff, families, and patients to 
promoted effective communication. A foundation of empirical evidence for the use of AAC to support the communication needs of patients with $\mathrm{CCN}$, whether temporary or permanent, has been laid. Moving forward, we must continue to develop education and training programs for healthcare staff, patients, and their families. Dr. Happ and colleagues have begun working with adult acute care nurses to assess the effectiveness of AAC education and training through hands-on education and CBLs (Happ, Baumann, et al., 2010). Additional research is needed to determine the most efficient method for knowledge and skill transfer with pediatric acute care nurses. A lack of research with pediatric nurses indicates a specific need for AAC education and training in the pediatric acute care setting. In addition to education and training, the development of functional $\mathrm{AAC}$ tools and strategies must continue as we seek to provide efficient communication access to all in need.

\section{Differences in AAC use with pediatric patients based upon unit}

Differing perspectives were found in the review of the pediatric literature based upon hospital unit and the type of patient potentially seen. Costello (2000) discussed being able to prepare patients, families, and nursing staff on the use of AAC before a planned surgery if the short-term loss of voice was anticipated. The outcomes and feedback of that study were positive from all parties involved (Costello, 2000); however, the implementation of AAC in the ICU when a patient was not prepared for admission, hospitalization, surgery, or speechlessness likely requires a different set of AAC methods and strategies based upon patient cognitive status, motor ability, attention, and nurse knowledge regarding AAC (Costello, L. Patak, and Pritchard, 2010). These situations that cannot be prepared for require proactive education and training.

Mesko et al. (2011) provided a short introduction to an AAC pain location-scale for pediatric patients undergoing tonsillectomy and adenoidectomy (T\&A). They found improved accuracy of pain location and demonstrated that the use of pictures post-surgery with temporarily non-verbal patients may be a beneficial way of easing the communication burden and improving nurse ability to provide quality care (Mesko et al., 2011). It is possible that these implementation studies in non-intensive care units may be more successful due to fewer time constraints and stable patient medical status. There is a need for additional research considering different hospital units and the ability to implement AAC strategies effectively across a variety of units and patients.

\subsubsection{Education with Nurses and Nursing Students}

Education is provided to students as well as practicing clinicians as healthcare provision, laws, and practices are ever changing. Research with social workers (Schinke et al., 1981) and nurses (Bissinger and B. Burns, 2018) has assessed the benefits, impact, and rigor of continuing education for practicing professionals. Research with students going into healthcare professions such as speech language pathology and medicine has demonstrated a variety of ways that communication skills may be taught using virtual and in-person 
methods with standardized patients or actual patients in clinical settings (M. I. Burns et al., 2012; Schinke et al., 1981).

Kruijver et al., 2000 evaluated a variety of communication training programs in nursing care and found that most found little to no change in nurses' skills and behavior nor patient outcomes, though this may have been related to weak study design. They did, however, find that research with nurse-minded teachers or educators showed more positive results in skill development, behavior change, and patient outcomes (Kruijver et al., 2000), supporting the role of participatory action research (PAR) in this study's development. Additional research has focused on the impact of communication education with specifically nursing students and found that it is needed often, both early on (first or second year of study) and later in education programs (fourth year) for maximal benefit to students (McCarthy, O’Donovan, and Twomey, 2008).

\section{Online education with nurses}

Online education has successfully provided information to adult learners such as nurses for the purpose of continuing education (Bissinger and B. Burns, 2018; Lahti et al., 2014; Lewis et al., 2001). However, certain aspects of clinical education may improve the experience and subsequent learning outcomes for participants such as ensuring the content is relevant, engaging, and includes examples or scenarios which have been found to assist in the transition from knowledge to clinical application (Lahti et al., 2014). Additionally, Nicoll et al. (2018) has raised awareness of the need for more rigorous study design and implementation with technology-enhanced learning like CBLs, which this research will attempt to meet.

Among the benefits of online or e-learning for healthcare professionals such as nurses are convenience, flexibility, ability to meet a variety of learning styles, and an adherence to adult learning principles (Gerkin, T. H. Taylor, and Weatherby, 2009; Billings, 2000; K. Campbell, V. Taylor, and Douglas, 2017). E-learning has also been found to be user-friendly with high participant satisfaction and similar educational and clinical benefits to in-person coursework (Billings, 2000). Kemper et al. (2006) found that the method of online education provision and delivery access (e.g., delivery by email or on a website and each delivery with small amounts of materials over time or lots of information in a short amount of time) did not impact participation or change in knowledge, confidence, or communication to the same extent that the inclusion of a small fee for continuing education units (CEUs) did. Participants who paid a small fee for CEUs were found to be three times more likely to complete the education, pointing to the inclusion a nominal fee as a motivator for many (Kemper et al., 2006).

\section{Online education with nursing students}

Nguyen (2015) conducted a review on the literature available on online education with nursing students, mostly at the undergraduate level. This assessment of the state of the science determined that most research shows that online learning appeared to be slightly more or 
equally effective compared to in-person education. However, with additional assessment, Nguyen (2015) found that many of the initial studies finding a mildly positive or neutral comparison between online and in-person education were not rigorously conducted, nor did they take into account selection bias. This points to a need for additional scientifically rigorous research in the area of online education effectiveness with nursing students.

Shorey et al. (2018) conducted communication education research specifically with first year nursing students in Singapore. Shorey et al. (2018) found that a blended learning pedagogy, including in-person and asynchronous distance education, was an effective way to teach communication skills to new nursing students with no clinical experience.

Communication between nurses and patients of all ages is essential for high quality patient care. The literature over the past 25 years continues to demonstrate a need for communication and AAC support for nurses and patients of all ages during hospitalization. AAC provides a breadth and depth of strategies, tools, and devices to assist in meeting those communication needs, whether temporary or permanent. This research provides a look at the state of the literature regarding AAC use by nurses with pediatric patients. Education and training regarding the importance and use of AAC in acute care settings should continue to be developed and studied so that we might equip nurses to serve patients through improved communication abilities resulting in quality care.

The current state of the literature demonstrates limited research on the education and training on, and implementation of, AAC with pediatric nurses for clinical use (Costello, 2000; Costello, Santiago, and S. W. Blackstone, 2015; Thunberg, Törnhage, and Nilsson, 2016); however, what exists indicates that AAC education and training benefits pediatric nurses and nursing students. The minimal research and gaps in supporting evidence prompted the development of this mixed methods research study including qualitative interview and focus group discussions followed by the development and implementation of education modules on AAC designed for pediatric nurses and nursing students.

\subsection{Theory}

This research was conducted using mixed methods, qualitative and quantitative data collection and analysis. In the field of speech-language pathology and communication disorders, qualitative research provides a depth and personal aspect of the data analysis process which might be missed with solely quantitative analysis techniques. As the goal of clinically-based speech-language pathology research remains improved clinical care and quality of life outcomes for patients and family members, qualitative and quantitative data analysis provides a well-rounded picture of current evidence, practice, and needs.

Research should have a solid theoretical basis and framework that informs the decisions and organization of data collection and analysis. This research began with the use of participatory action research (PAR) which utilized the input and experiences of the target populations: pediatric nurses, and nursing students. Qualitative data was collected and 
analyzed using grounded theory and thematic analysis. This information was used to delve into the theoretical underpinnings of educational practice which continues to evolve with a recent focus on adult education. This research included the development and dissemination of educational modules on communication and AAC, developed using the framework of Gagné's cognitive instructional theory and conditions of learning as well as Bandura's social cognitive theory (Bandura, 1977b; Gagné, 1975; Gagné, 1975; Gagné, 1984).

\subsubsection{Participatory Action Research}

PAR includes the target population or 'consumer' in the development of the research questions, implementation of the study, and interpretation of the findings. This approach focuses on collaboration between researchers and consumers; pediatric nurses and nursing students for this research. This inclusion of 'consumer' populations has been shown to increase the relevance and social validity of the research (Schwartz and Baer, 1991; White, Suchowierska, and M. Campbell, 2004; M. M. Wolf, 1978). In addition, some research has shown that PAR may lead to more user friendly tools, sustainability of programs and outcomes, accepted interventions, focused and clinically relevant research questions, and beneficial dissemination practices (O'Fallon and Dearry, 2002; White, Suchowierska, and M. Campbell, 2004).

This was accomplished in this research endeavor through continual inclusion of pediatric nurses and nursing students in all phases of development and implementation. Nurses were active in the development and execution of focus group and individual interview discussions, the piloting of the education modules, and provision of specific feedback prior to the final data collection trial. The goal of using this approach to develop and carry out this research is to improve both science and practice $(\mathrm{H}$. R. Turnbull and A. P. Turnbull, 1991).

\subsubsection{Grounded Theory and Thematic Analysis}

Qualitative data analysis includes an often iterative and flexible process when compared to the analysis of quantitative data. Data was collected through the lens and frameworks of PAR and grounded theory. Grounded theory methodology was originally described by Glaser and Strauss (1967) and includes a process of collecting data then coding, analyzing, and interpreting that data through ongoing analysis. According to this methodology the data collection process and method must be considered in the interpretation process as well as the actual data collection itself. This data analysis process requires the person collecting the data be involved in all aspects of data collection, analysis, and interpretation. This research process included: memoing (providing a general outline of content in a more concise narrative), open coding (consolidating the data from the previous stage), axial coding (categories of information derived from open coding), and finally theme development (Flick, 2014; Charmaz, 2006). 
This research also incorporated aspects of thematic analysis into the four-part data analysis process. The inclusion of this method of analysis allowed the comparison across groups; for this research, pediatric nurses and nursing students. Thematic analysis follows a six-step process which included the researchers familiarizing themselves with the data, generating initial codes, searching for themes, reviewing themes, defining and naming themes, and producing a report (Braun and Clarke, 2006; Flick, 2014). There is clear overlap in the four-phase process of grounded theory and the six-steps of thematic analysis which helped to outline how the PI and research assistants interacted with, digested, and distilled the qualitative data. Namely the memoing phase and familiarization with the data, and the open coding and initial code generation were conceptually the same steps or phases. The axial coding happened alongside searching for themes, theme development, and theme review and definitions simultaneously.

Both of these theoretical frameworks assist in ensuring the qualitative data processing remains methodical and organized throughout analysis. The final product is significant research data distilled to overarching themes and sub-themes that provide a 10,000 foot view of the information in a way that can be used. The goal of this research was to learn from pediatric nurses and nursing students regarding their experiences and preferences regarding continuing education on AAC and its clinical relevance. Thus, the themes which emerged from the qualitative data assisted in the development and organization of educational modules with those two populations, pediatric nurses and nursing students, in mind.

\subsubsection{Gagné's Cognitive Instructional Theory}

Cognitive instructional theory outlines a way of considering how we learn new information and how we then generalize that information into practice across multiple situations. Gagné's framework, grounded in the information processing model, includes a process for learning information as well as conditions of learning. Five categories of learning and nine 'events of instruction' are outlined to provide structure in how information is taught to a learner (Gagné, 1975). Gagné's conditions of learning provide a structure for considering what is being taught, under what conditions, and to whom, with hopes of understanding and applying the most effective educational method. This theory also strongly encourages the transfer of information that may generalize across contexts and other 'defining' factors such as age, gender, or race, to name a few.

\section{Processes of learning}

Gagné (1975) outlines the conditions of learning hierarchy including eight phases:

1. Expectancy or the initial motivation

2. Attention and selective perception or the apprehending phase

3. Acquisition or coding and storage entry

4. The retention phase, moving information into memory storage 
5. Retrieval of information during the recall phase

6. Generalization or transfer

7. Performance or responding phase

8. Feedback or reinforcement phase

These phases of learning should be taken into consideration when assessing an education framework to systematically provide information and proper opportunity to maximize learning for individuals. It must be considered that certain parts of the learning process are external to the learner while others are internal. Learning is inferred when a change can be identified in an individual's behavior. Though this research will be unable to measure that sought after change, it will measure self-efficacy, as discussed later in this chapter, to attempt to gather whether participants are more comfortable using communication strategies, therefore more likely to do so in practice.

Gagné (1975) discusses eight phases that occur when new information is learned, mentioned above and expounded upon here. For something to be learned, there must first be motivation by the learner, either through an incentive driving the learner to learn or through expectation of the benefit of the learning act. We attempted to ensure that in this research through PAR where pediatric nurses and nursing students provided insight and expertise on content relevance. It is then required that a learner attend to the new information and then perceive the information provided. This has been achieved by creating succinct online education modules with auditory input, text, and case studies. The third 'phase' or acquisition phase is where learning actually begins to occur. New information is encoded and stored in short-term memory which has been targeted through the multimodal method of information presentation just mentioned. This is followed by the retention phase where that information moves to long-term memory where it becomes more or less permanent or solidified. This can be assessed through the recall phase in which stored information must be brought to attention in order to be carried out or used. Retention and recall have been targeted through the development of pre- and post-test questions to assess whether participants are able to recall information from module to module; however, due to the nature of this study, long-term retention and recall will not be assessed.

Retrieval of information can be cued by external factors such as a key words, a physical handout, or a reminder. This research provides all participants with summarized handouts of the information covered as a reference as well as sending them low-technology communication boards they can use in their clinical work. The next phase includes the generalization of information which we hope occurs, though requires a transfer of knowledge into a new context. The performance phase allows the learner the chance to demonstrate what they learned and the final phase is the elicited feedback and reinforcement of an onlooker, teacher, or even the learner themselves. Though the nature of this research does not include the opportunity to see how participants practically enlist what they have learned, it does seek to measure their perceived ability to use the information presented through self-efficacy measures for each module. 


\section{Categories of learning}

In addition to the eight phases or conditions of learning, Gagné (1984) outlines five categories of learning: verbal information, intellectual skills, cognitive strategies, motor skills, and attitudes. We seek to provide information using these categories of learning, hoping for generalization outside of the 'taught' or specific 'practiced' scenario. This is one of the initial steps in developing education as it helps in determining types of learning outcomes. Verbal information, also called declarative knowledge, includes information that can be repeated back either verbatim or through a summary of the content. This type of knowledge is often transferred through school or learning environments and provides us with the knowledge base needed on a topic or subject. This type of knowledge will be a major target area in the implementation of the CBLs in the current research and assessed through the use of pre-test and post-tests which has been deemed effective in previous nursing research (Egerod et al., 2020; Keough and Tanabe, 2011). The knowledge outcome in this research aligns with the second portion of Gagné's definition for evidence of learned declarative knowledge which states that a learner will be able to "reconstruct an organized representation of a verbal passage, containing identifying main and subordinate ideas arranged in a meaningful schema" (Gagné, 1984 p.380).

Intellectual skills may include concepts, procedures, and rules, such as the rules of language and the way they are applied through written or spoken communication. These 'procedural rules' are discussed in the education modules created for this research; however, they will not be directly assessed or measured in the current line of study (Gagné, 1984). Intellectual skills may not be easily assessed in outcome measures because of the progressive nature of acquisition.

Cognitive strategies or problem-solving skills can be specific or general but provide the learner "some degree of control over the processes involved in attending, perceiving, encoding, remembering, and thinking" (Gagné, 1984 p. 381). These skills or strategies are taught so that learners can use and adapt them based on their situation. For the purpose of this research, we will target strategies and skills for the use of no-technology and lowtechnology AAC in clinical settings. Targeting of these strategies or skills were attempted in the CBLs through the inclusion of handouts, vignettes or case study examples, and decision making trees to provide pediatric nurses and nursing students with problem-solving tools to use with their pediatric patients with $\mathrm{CCN}$.

The vignettes, in particular, will provide a model, which we know may be a primary method of learning (Bandura, 1977b). For this to take place, the learner must recall pertinent rules that they are familiar with and then take that information and add to it in the learning process. The external conditions of learning for cognitive strategies often include verbal instructions to guide the learners' thinking. In addition, participants will be given the opportunity to receive physical low-technology communication boards, as seen in the education modules. These cognitive strategies or executive control processes help learners bring past knowledge and experiences into their current learning situation and build upon it to enable them to attend, perceive, encode, remember, think, and hopefully apply new 
information (Gagné, 1984). This research leans on the known understanding that cognitive processes or intellectual skills play a primary role in the development of novel behaviors in learners (Bandura, 1977b).

Motor skills are evidenced by a steady improvement in the quality of a movement that is typically achieved with much repetition. This often looks like improvement in the smoothness or timing of a movement or action over time as a new motor plan is laid and a skill is acquired. This current research does not address the development of a motor skill, though future research with AAC may benefit from the consideration of all five categories in developing functional AAC users and supporters of communication. This research does, however, include the use of the psychomotor scale within the L-SES (Kang et al., 2019) which provides a measure of perceived comfort completing a task or carrying out a newly learned skill.

The final category of learning is attitude. This type of learning is much harder to target and develop as our attitudes are so closely tied to our emotions. Our attitudes do not always serve as a predictor of our behavior or actions and thus can be difficult to shape or change through common educational models (Gagné, 1984). This research attempted to measure participants' self-efficacy and attitudes toward communication strategies, education effectiveness, and general AAC knowledge. Attitudes are very complex, multi-faceted in nature and according to Gagné's theory, include three facets, cognitive (ideas or opinions about things), affective (emotion or feeling related to an idea or opinion), and behavioral (the readiness to act on an idea or opinion; Gagné, 1984). This will be measured using a validated self-efficacy tool which looks at each of these facets of perceived comfort using presented information (Kang et al., 2019).

Oftentimes attitudes are formed from interactions we have with others or may be formed over several years based on our experiences. There are three general ways that attitudes are changed including classical conditioning, perception of success in behavior, or human modeling (Gagné, 1984). Kang et al. (2019) validated a learning self-efficacy scale (L-SES), created specifically for assessing perceived success with taught clinical skills. Internal conditions to consider when attempting to change attitudes include the concept of this type of information and at least some prerequisite knowledge of the topic (Gagné, 1984). The external conditions that must be provided in the education (ex., vignettes and case scenarios) are an opportunity to see a human model and variations on that model as reinforcement of the information presented (Gagné, 1984).

These five categories of learning are described by Gagné (1984) as distinctive and yet areas of overlap. We can target multiple categories of learning in education, and it may be argued that we should in order to solidify information and learning in a multi-modal fashion. 


\section{Conditions of learning}

Gagné purports that learning is assumed when there is a change in ability over time that cannot be the result of growth (Gagné, 1984). Thus, this section plums the conditions or circumstances under which learning might best take place based upon each of the previously mentioned categories of learning targeted in this research (verbal information, intellectual skills, cognitive strategies, and attitudes). It is important to note that these are two-part conditions including both the condition(s) of the learner and the learning situation.

In Gagné's book entitled The Conditions of Learning, (Gagné, 1984) he discusses the learning of verbal information which includes labeling and facts. The internal learner characteristics include a previously created set of organized knowledge to draw from and build upon, and successful strategies for long-term encoding or storing information. External conditions provide a meaningful context to add new information to throughout the learning process, increasing the memorability of information presented, and repetitively engaging with information to help commit it to memory.

Gagné discussed intellectual skills in two chapters of his book, The Conditions of Learning, outlining four aspects of intellectual skills (Gagné, 1984). Gagné begins with the learners' need to discriminate new information as the ability to recall and differentiate between terms or information. The learning situation must in turn provide feedback and reinforcement of correct vs incorrect responses. For the learning of concrete concepts, Gagné proposes that a learner must have pre-established discrimination which can be recalled when learning new concepts. The situation provides a set of verbal cues which prompt the learner to engage with information in ways that develop discrimination, generalization of information, assessment of knowledge, and reinforcement. Defined concepts are discussed next as, "a rule that classifies objects or events" such as a definition (Gagné, 1984, p. 129). The conditions for learning outlined for this type of information include a learner who can access their working memory to use the new information. The learning situation must clearly present the information to be learned either in spoken or written form for the learner to engage with. The final aspect of intellectual skill to be learned is rule learning which requires a learner who understands what a rule is in general and can then bring in a new rule (a predetermined but unstated guideline) to the previously developed schema. For a learner to learn a rule, the situation must provide verbal instructions which may be probed by the instructor and reinforced for learning to occur.

Coulter, 1990 examined the use of Gagné's conditions of learning and theory of instruction (Gagné, 1985) compared to a humanistic approach to learning as outlined by Rogers, 1983 for nurse education. Coulter, 1990 argues that each type of learning has a role

and may be beneficial based upon the situation. For the purpose of this research including online education modules, it was determined that a more behaviorist style of teaching and learning, as found in Gagné's theory, was most appropriate. 
Table 2.1: Use of Gagné's Events of Instruction in This Research. ${ }^{1}$

\begin{tabular}{ll}
\hline Gagnés Events of Instruction & $\begin{array}{c}\text { Inclusion in "AAC Education for Pediatric } \\
\text { Nurses and Nursing Students" }\end{array}$ \\
\hline $\begin{array}{l}\text { Gaining attention } \\
\text { Informing the learner of the objective }\end{array}$ & $\begin{array}{l}\text { Opening introduction. } \\
\text { Informal learning objectives provided at the } \\
\text { beginning of each module. }\end{array}$ \\
Stimulating recall of prerequisite learning & $\begin{array}{l}\text { Discussing previous material or background } \\
\text { knowledge through review and recap at the } \\
\text { end of each module. }\end{array}$ \\
Presenting the stimulus material & $\begin{array}{l}\text { Education of new information with use of } \\
\text { auditory information, orthographic text, and } \\
\text { vignettes with use of new information. }\end{array}$ \\
Providing learning guidance & $\begin{array}{l}\text { Strategies, decision-making tree, and } \\
\text { vignettes. }\end{array}$ \\
Eliciting the performance & $\begin{array}{l}\text { Post-test including Learning Self Efficacy } \\
\text { Scale (L-SES). }\end{array}$ \\
Providing feedback & $\begin{array}{l}\text { Post-test feedback. } \\
\text { Assessing change from pre- to post-test } \\
\text { regarding knowledge and self-efficacy. }\end{array}$ \\
Rssessing the performance & $\begin{array}{l}\text { Retention questions and review in later } \\
\text { modules. Handouts provided after } \\
\text { completion of the education modules. }\end{array}$ \\
\hline Enhancing retention and transfer &
\end{tabular}

${ }^{1}$ Source: Gagné, 1975

\section{Events of instruction}

Instructional design considers two main factors: the learner and the objectives. The nine events of instruction laid out by Gagné (1975) and Gagné (1984) provide a practical framework, based on the information processing model, for designing and carrying out education in a systematic and evidence-based manner. This theory has been trialed in multiple sectors including the military, aviation, leadership, and healthcare (ex., Khadjooi, Rostami, and Ishaq, 2011; Buscombe, 2013; Wong, 2018). Table 2.1 explains how each of the nine events of instruction have been incorporated in the steps and development of the education modules in the current research (Bandura, 1977b; Gagné, 1975).

The previously discussed conditions of learning are essential to this instructional theory as well as internal (previous knowledge) and external conditions (what is being taught). The previously mentioned categories of learning are important to determine prior to developing education material in order to structure the information design and presentation appropriately. 
Gagné's nine events of instruction have been used by a variety of educational programs in recent years to create a more systematic approach to knowledge exchange (ex., Buscombe, 2013). Among these are an undergraduate nursing program who hoped to improve student outcomes and teacher effectiveness through adopting Gagné's framework for instruction (Miner et al., 2015). Their implementation demonstrated improved student ratings of the instructor's perceived preparedness, mastery of content, enthusiasm, and overall effectiveness over three semesters (Miner et al., 2015). Students also reported a higher likelihood that the course impacted the development of their critical thinking skills (Miner et al., 2015). A significant change in students' grades was not evidenced; however, the overall range of grades did diminish with a higher low-end course grade (Miner et al., 2015).

As mentioned before, for the current research not all five types of learning are expected to take place. This research will focus mostly on verbal information, intellectual skills, cognitive strategies, and attitudes through the focus on knowledge exchange with the inclusion of education, handouts, role-play depictions, and decision trees.

\subsubsection{Bandura's Social Cognitive Theory}

Bandura's social cognitive theory proposes that individuals interact in a mutual twoway exchange with their environment and behavioral responses to cause change in an individual through life experiences. According to this theory, a person's characteristics such as genetics, knowledge, values, and beliefs impact their behaviors; however, these behaviors may include social interactions and reactions which also may affect behavior. In addition, the environment which may include social interactions may change how we act, or aspects of our personalities or behaviors may elicit changes in our personalities via feedback received. Bandura terms this interplay between one's environment, behavioral responses, and life experiences as "triadic reciprocal causation" (Films Media Group, 2003; Bandura, 1998).

\section{Modeling and observational learning}

Experiential learning is a part of the learning process especially when developing a new skill; however, we are not always expected to demonstrate or display every piece of knowledge that we obtain. Experiential learning has benefits, but may come with a series of drawbacks such as the potential for danger depending on the experience you are learning from. In pediatric acute care, there are huge safety implications for someone learning something new that may impact someone's health. The use of experiential learning with an instructor present is often time consuming and detracts from nurses' other duties while at the hospital and therefore may not prove practical or economically responsible.

Per Bandura's social cognitive theory (Bandura, 1977b; Bandura, 1986; Bandura, 1998), humans have the ability to learn via observational learning. Observational learning includes four processes: attention, symbolic representation, transformational action, and 
motivational incentive. Attention requires that learners pay attention to what is being taught and modeled to glean the important information. They then must comprehend and remember that information (symbolic representation) followed by determining what they should do with that information (transformational action) and then actually be motivated to do something with what they have learned (motivational incentive; Bandura, 1977b; Bandura, 1986; Bandura, 1998).

Bandura's theory has been tested and utilized as a developmental framework across many fields, including the healthcare and nursing fields (Ziegler et al., 2005), making it an appropriate match to the current line of research. This social cognitive theory has provided the structure of modeling and reinforcement to teach new information while building the self-efficacy of the individual learning (Bandura, 1977a; Ziegler et al., 2005). Bandura discusses social modeling, social persuasion, self-efficacy, and physical and emotional states all playing a large role in the effectiveness of a learner mastering new information.

\section{Self-efficacy}

Bandura states that self-efficacy, believing that you can do something through the experience of doing it, is essential to effective learning and resulting carryover of what you have learned (Bandura, 1977a; Films Media Group, 2003; Ziegler et al., 2005; Bandura, 1998). Social modeling is an important aspect of learning and the development of self-efficacy according to Bandura (1977a) and Bandura (1998), entails watching others successfully complete an action or task and being encouraged that the new learner could do the same. Someone motivated by someone else's faith in their abilities is interacting with social persuasion, while physical and emotional states may also be determining factors in how effectively you learn to and successfully do something.

Self-efficacy, is defined by Bandura (1977a) as a perceived confidence in performing an action or behavior. Bandura (1977a) discusses efficacy expectation (feeling one can complete an action or behavior to achieve an outcome) vs outcome expectancy (one's perception that a behavior will lead to an outcome). He argues that efficacy expectation may be more of a determining factor than outcome expectancy as to whether someone will attempt a new action or behavior. The reasoning follows that you may believe an action will result in an outcome, but if you are not confident you can perform that outcome, you are not likely to attempt it (Bandura, 1977a).

Bandura (1977a) and Bandura (1977b) proposes four sources of efficacy expectations: performance accomplishments, vicarious experience, verbal persuasion, and emotional arousal. Due to the nature of this computer-based education research, performance accomplishments and emotional arousal were not measured. This research primarily targeted self-efficacy through vicarious expectations using modeling of scenarios and verbal persuasion encouraging participants in their ability to use no- and low-technology AAC. Vicarious expectations have been found to be beneficial, especially if models are conducted with a clear goal or outcome as has been targeted in this research. Research by Bandura (1977a) 
supported self-efficacy as a predictor of task performance regardless of the efficacy source and found a positive relationship between efficacy expectation and performance.

Self-efficacy was measured in this research to infer participant comfort and predict potential ability to use taught information clinically. This was done using a pre- and posttest measure of self-efficacy on a $100 \mathrm{~cm}$ sliding scale. The prompt included a definition of self-efficacy and a prompt to, "Please move the slider on the scale to indicate your level of self-efficacy regarding your ability to use no-technology AAC." The prompt differed only regarding the specific content or action targeted for each education module.

Learning self-efficacy, a post-test measure included in this research, was defined by Kang et al. (2019) as, "learners' confidence in their capability to learn specific subjects." Kang et al. (2019) created and validated a clinical tool entitled the learning self-efficacy scale (L-SES) for use with clinicians. In addition to the validated L-SES, this research has included a pre- and post-test assessment using a sliding scale where participants can indicate their perceived ability to use the particular strategy/task for each of the education modules. In addition, learned self-efficacy was measured using Kang et al. (2019) L-SES in the post-test only.

Our efficacy beliefs impact us in four main areas: cognition (whether we believe we can), motivation (whether we strive to accomplish our goals), emotion (how we feel about our goals and abilities), and decision-making (making decisions based on our perceived abilities; Films Media Group, 2003). This research process takes into consideration each of these areas by including opportunities for learners to improve their perception of their knowledge, purpose, and abilities in making decisions about AAC to improve the quality of life for their patients and themselves during acute medical encounters. 


\section{Chapter 3}

\section{Methodology}

A mixed methods approach was used in this research, gathering both qualitative and quantitative data. Qualitative data will provide in-depth information regarding nurse knowledge and recommendations regarding augmentative and alternative communication (AAC) which is most pertinent to the computer-based learning modules (CBLs) being developed. Quantitative data was collected from pre- and post-assessments then analyzed and compared across modules and groups.

This research was guided by the following research inquiries (RI) with data collection discussed in this chapter and proposed hypotheses, analyses, and results discussed in Chapter 4:

RI-1. What content do pediatric nurses and nursing students find relevant to their clinical work?

RI-2. Based on pre- and post-test results from the computer-based learning modules, how do pediatric nurses and nursing students':

a. Knowledge of AAC change?

b. Self-efficacy using AAC change?

RI-3. Based on post-assessment results from the CBLs, how do pediatric nurses and nursing students rate the:

a. Adapted Learning Self-Efficacy Scale (Cognitive, Affective, Psychomotor)

b. Relevance of Content

c. Effectiveness of the Educator and Method

\subsection{Focus Groups and Interviews (RI-1)}

Focus groups are a tried and true qualitative data collection tool used for purposes such as gathering users' feedback for improvement of a product. Focus groups have been demonstrated to be effective in the development and refinement of instruments such as 
education modules. Thus, they were employed to gather robust qualitative data from small groups of pediatric nurses and nursing students. In addition, individual semi-structured interviews were used in instances of scheduling difficulties when multiple nurses or nursing students were not available to participate in a focus group synchronously.

A focus group and semi-structured interview outline were utilized to guide discussion through the potential topics covered in the CBLs. These topics were discussed and aspects of each were prioritized in the CBL development based on participants' perception of relevance, overall importance to everyday practice, and access to differing communication supports.

Per common recommendation (Flick, 2014), each focus group strove to contain five to eight pediatric acute care nurses or nursing students; however, due to scheduling barriers and the public health crisis resulting from the COVID-19 pandemic, this was not always possible. One focus group of pediatric nurses contained five nurses and a focus group with nursing students contained two participants. In addition, five pediatric nurse interviews and three nursing student interviews were completed. Each group and individual interview discussion covered the same topics including the basics of communication, AAC in general and specifics, and low-tech, mid-tech, and high-tech AAC. Clinical access to these types of communication as well as their familiarity and comfort using them were discussed. Audio information gathered from the focus groups and interviews was collected and stored on the PI's password protected computer. The data was word for word transcribed and thematically analyzed using grounded theory and thematic analysis (Glaser and Strauss, 1967).

Focus groups and interviews were scheduled based upon participant availability. Focus groups and interviews took place in person or via voice over internet protocol (VoIP) such as Zoom (Barbu, 2019), which allowed audio recording of the interactions for later transcription and qualitative analysis. The location was agreed upon or method of communication (ex., Zoom) determined by participants and the researcher to ensure convenience for all parties. Each participant was provided with an informed consent form prior to beginning data collection with ample time to read and determine their ability and desire to participate. Following the signing of informed consent documents, participants were offered a general outline of the topics and information to be covered during the focus group or interview. The primary investigator (PI) engaged participants in a 45-75 minutes discussion of relevant communication strategy and AAC topics to determine nurse and nursing student priority regarding information they have and information that they want vs need to serve their patients well in acute care settings.

Fifteen participants were a part of the qualitative interview or focus group data collection process. Five nursing students representing three nursing programs in Tennessee and ten pediatric nurses representing four total pediatric hospitals in Tennessee and California. Three individual interviews and one focus group was conducted with nursing students, and five interviews and one focus group was conducted with pediatric nurses. 
One pediatric nurse was included in the pediatric nurse group as she is currently working in that capacity while finishing her pediatric nurse practitioner degree.

Following data collection, each focus group and interview was word for word transcribed by hand and analyzed using tenants of grounded theory and thematic analysis with NVivo (QSR International Pty Ltd., 2019). Thirty-three percent of the transcribed data was reliability checked by another researcher in the UT-AACL and demonstrated $>99 \%$ transcription reliability. The PI coded each focus group and interview transcript through an iterative process of coding based upon grounded theory and thematic analysis. A fellow researcher in the UT-AACL provided inter-coder reliability across $25 \%$ of the transcript data with initial overall agreement $>95 \%$. The inter-rater reliability coding included comparison of initial thematic coding discrepancies and discussion until complete agreement was reached. Minimal discrepancies were identified, with all discussed and agreement achieved between researchers to identify the final themes and sub-themes for this data-set. The resulting themes and sub-themes are discussed in detail in Chapter 4 and Chapter 5.

Focus group and interview participants had the opportunity to provide the PI with their email address and name to enter a drawing for one of eight $\$ 10$ Amazon or Visa gift cards as a thank you for their participation in a focus group or interview. Eight participants were randomly identified and emailed with a digital gift card of their choice (Amazon vs. Visa) by August 2020.

\subsection{Computer-Based Learning Modules (RI-2 and RI-3)}

\subsubsection{Previously Developed CBLs}

Four, 30-minute CBLs were created using mixed methods data (survey and interview data) from previous research with pediatric nurses. Data collected from local pediatric acute care nurses were analyzed and used to develop themes and topics for CBL modules. The PI met with UTK-CON faculty (academic and clinical) to discuss content areas and the overall organization of the CBL modules. Additionally, multiple meetings with staff in Organizational Development and Learning (ODL), nurse managers, and unit nurses at a local pediatric hospital were conducted resulting in the request for four 30-minute education modules as opposed to the original proposal of three longer modules.

Content development took place over three months (03/2018 - 06/2018) and included input from academic and clinical nursing faculty and pediatric nurses in out-patient neurology, post-operative surgery, and a nurse who floats across all units at a pediatric hospital. Each of these individuals participated in a 30-60-minute discussion covering content feedback and development for each of the four modules. They provided verbal feedback and advice on appropriate content for the modules, terminology that was understood in the pediatric nursing realm, advice on the order and organization, and feedback on making the CBLs engaging, succinct, and clinically relevant. Two of the three nurses were given a preview of the videos when under development, and they provided specific 
feedback on things to be removed or changed. Final review of the videos was conducted by the primary investigator (PI), academic faculty in the Audiology and Speech Language Pathology program, and individuals from ODL at a local pediatric hospital.

All information received during meetings and discussions were incorporated into the final CBLs, portions of which were piloted with four pediatric acute care nurses from a single local pediatric hospital. All piloting of these education modules indicated that the CBL format was appropriate; however, pilot participants reported that the modules were too lengthy and there was a lack of access to communication tools and supports discussed, therefore, rendering the CBLs somewhat irrelevant to their clinical work.

A small feasibility pilot of these CBLs was conducted with nursing students and speech-language pathology students as well. Limited participation from these two groups yielded inadequate results to draw conclusions that would generalize. Thus, it was determined that additional CBL development, piloting, and trailing was indicated.

\subsubsection{Development and Preliminary Piloting of New CBLs}

The previous piloting of four, 30-minutes CBLs with four local pediatric nurses indicated positive learning outcomes but participants reported the modules were too long and that some content was not relevant to their clinical work (e.g., they did not have access to midor high-tech AAC). Researchers sought to improve upon the former education modules by conducting focus group discussions and individual interviews with pediatric nurses and nursing students locally and from a more diverse geographic swath including multiple locations across Tennessee and in California. The qualitative data were analyzed, thematically coded, and used to inform the development of more focused and efficient CBLs on communication and AAC for pediatric acute care nurses and nursing students. Themes and sub-themes emerged from the qualitative data and were agreed upon by the PI and a research assistant. This information was listed and referenced during the development of the education modules to ensure recommendations and themes identified were included in each module. Pre- and post-test questions (five to six questions for each pre-test with the same questions repeated for the post-test), found effective in nursing research (Egerod et al., 2020; Keough and Tanabe, 2011), were developed based upon information highlighted in the qualitative data collection and analysis and ensured to be covered in the CBLs, as a written script was developed for each module prior to filming and piloting. The pre- and post-test questions included multiple choice, multiple option, open-ended, and true false formats. CBL content was filmed and edited by the PI and Nathan Simmons.

Each of the three CBLs included a teaching portion including new terminology and information regarding $\mathrm{AAC}$, one to two easily implementable communication strategies, vignettes and scenarios acted out using each strategy, and a recap of the information covered. The first module included a basic introduction to AAC including vocabulary such as receptive and expressive communication and communication in general. Module 1 included the no-technology strategies of wait time and rephrasing/recasting. Module 2 provided information on the four main categories of AAC and introduced the variety in 
size, shape, and organization that is found in low-technology communication boards. This module then provided instruction on ways that healthcare providers can provide choices such as with the use of physical items, pictures, or a communication board. Additional discussion included no-technology yes/no options such as thumbs up/down, eye blinks, and simple sign language that might be used in the acute care setting. The final module included instruction on what to take into consideration when attempting communication with a patient with complex communication needs. This module focused on teaching partner-assisted scanning. A fourth optional module provided instructions and a how-to video for the creation of personalized low-technology communication boards. All materials discussed were summarized and provided following participation in the research, including the template file for low-technology communication board creation.

Following the creation of the new CBLs, four local speech-language pathologists reviewed the pre- and post-test questions (Appendix B), written scripts for the CBLs, and watched and provided feedback on the videos themselves. Once their feedback was incorporated into the pre- and post-tests and the educational videos, the CBLs were piloted with five piloting participants ( 3 pediatric acute care nurses and 2 nursing students). This piloting phase was included to reduce the potential for technical difficulties and for increased validity, ensuring that the wording and information was clear.

Five individuals who had participated in the qualitative focus group and interview portion of this research indicated a willingness and interest in participation in additional piloting of the education modules related to this research. Three pediatric nurses and two nursing students were a part of the piloting of the pre- and post-test questions surrounding three short education modules on communication and no and low-technology AAC. Their results pertaining to R-2 and R-3 are described here with the level of statistical significance identified at $p \leq 0.05$.

RI-2. Based on pre- and post-test results from the computer-based learning modules, how do pediatric nurses and nursing students':

a. Knowledge of AAC change?

Pilot participants $(n=5)$ demonstrated a positive knowledge change from pre- to posttest for modules 1 and 3 that was statistically significant (see Table 3.1). Module 2, however, demonstrated sightly lower scores on the post-test compared to the pre-test scores $(p=.374)$. For module 2 , four of the five participants scored the same on the pre- and post-test with a single participant scoring one point lower on the post-test than the pre-test. All five participants scored an $86.67 \%$ or higher on the pre-test at the outset, which may be due to all five pilot participants having some familiarity with the content from their participation in the qualitative data collection (interviews and focus groups), all of which included discussions and opportunities for learning about the module content.

b. Self-efficacy using AAC change? 
Table 3.1: Pilot Pre- to Post-Test Scores.

\begin{tabular}{lcccccccc}
\hline $\begin{array}{l}\text { Module } \\
\text { Test Scores }\end{array}$ & Mean & SD & SE & $\begin{array}{c}\text { 95\% } \\
\text { CI Lower }\end{array}$ & $\begin{array}{c}\text { 95\% } \\
\text { CI Upper }\end{array}$ & $t$ & $d f$ & $\begin{array}{c}\text { Sig. } \\
\text { (2-tailed) }\end{array}$ \\
\hline Module 1 & -2.80 & 1.64 & .74 & -4.84 & -.76 & -3.81 & 4 & .02 \\
Module 2 & .20 & .45 & .20 & -.36 & .76 & 1.00 & 4 & .37 \\
Module 3 & -1.20 & .84 & .37 & -2.24 & -.16 & -3.21 & 4 & .03 \\
\hline
\end{tabular}

There was an increase in self-efficacy from pre- to post-test for all three modules, however, the analysis did not reach statistical significance. This was likely due to the smaller sample size of these variables as four of the five participants completed the self-efficacy measure in the pre- and post-test for module 1 and only three of the five did so for modules 2 and 3. Despite not achieving statistical significance, all self-efficacy mean scores increased from pre- to post-test anywhere from 22 to 46 points. It is likely that with a larger sample size, this paired t-test measurement would have achieved statistical significance for all three modules.

RI-3. Based on post-assessment results from the CBLs, how do pediatric nurses and nursing students rate the:

a. Adapted Learning Self-Efficacy Scale (Cognitive, Affective, Psychomotor)

b. Relevance of Content

c. Effectiveness of the Educator and Method

For all three measures conducted in the post-test only, reliability was assessed prior to additional statistical analyses. Pilot data reliability can be found in Table 3.2 For the purpose of the pilot, all five participant's data was analyzed as one group, therefore no additional statistical analyses were run. Descriptive results were conducted and are discussed below.

Table 3.2: Pilot Post-Data Reliability.

\begin{tabular}{lccccc}
\hline Module Reliability & Cognitive & Affective & Psychomotor & Relevance & Effectiveness \\
\hline Module 1 & $\alpha=.78$ & $\alpha=.90$ & $\alpha=7.446 \mathrm{E}-14$ & $\alpha=.72$ & $\alpha=.86$ \\
Module 2 & $\alpha=-9.474 \mathrm{E}-14$ & $\alpha=.80$ & $\alpha=.71$ & $\alpha=.60$ & $\alpha=1.543 \mathrm{E}-12$ \\
Module 3 & $\alpha=1.00$ & $\alpha=-.19$ & $\alpha=1.00$ & $\alpha=.73$ & $\alpha=-.29$ \\
\hline
\end{tabular}

Each of the three subsets of the Learning Self-Efficacy Scale (L-SES) were initially assessed for reliability using Cronbach's alpha for each module. All three scales demonstrated good reliability as determined by a Cronbach's alpha of greater than .70 in two out 
of three modules. The cognitive and affective L-SES scales included four items. Modules 1 and 3 of the psychomotor scale included 4 items and module 2 included 6 items. The relevance scale had 7 items and the effectiveness scale had 8 items for all three modules. Each scale had a module where the reliability score was difficult to interpret or appeared to be very small, though it was a different module for each measure. This may be due to the small sample size for this pilot, due to a lack of variability in the data, or due to limited reliability of the scale. Upon further examination, it appears there was limited variability for the psychomotor scale in module 1 , cognitive and effectiveness scales in module 2, and the effectiveness scale in module 3 . The relevance scale in module 2 and the affective scale in module 3 did appear to have some variability, however, there were only five participants in this pilot. The reliability of each of these scales will be further analyzed in the full data set.

The average scores for each module and scale can be found in Table 3.3. All scale averages across pilot participants for each module indicated Likert scale values of strongly agree for all scales other than the affective L-SES scale, comparing this education to other continuing education completed, which indicated an overall rating of somewhat agree. This indicates a perceived confidence and self-efficacy regarding new knowledge learned, the ability to use that knowledge, and perceived relevance of content and effectiveness of the education method. The affective sub-scale of the L-SES may have been harder for participants to 'score' because of the vague comparative nature to other continuing education. Nursing students, for example, may not have continuing education experiences to compare this to; however, as this three part scale has been validated for use with healthcare workers, all three sub-scales were retained for the full project implementation.

Table 3.3: Pilot Post-Test Scale Averages.

\begin{tabular}{llll}
\hline Scale & Module $\mathbf{1}$ & Module 2 & Module 3 \\
\hline L-SES: Cognitive & Strongly agree & Strongly agree & Strongly agree \\
& $\bar{x}=4.67$ & $\bar{x}=4.95$ & $\bar{x}=4.80$ \\
L-SES: Affective & Somewhat agree & Somewhat agree & Somewhat agree \\
& $\bar{x}=3.55$ & $\bar{x}=3.55$ & $\bar{x}=3.65$ \\
L-SES: Psychomotor & Strongly agree & Strongly agree & Strongly agree \\
& $\bar{x}=4.80$ & $\bar{x}=4.87$ & $\bar{x}=4.80$ \\
Relevance & Strongly agree & Strongly agree & Strongly agree \\
Effectiveness & $\bar{x}=4.71$ & $\bar{x}=4.60$ & $\bar{x}=4.57$ \\
& Strongly agree & Strongly agree & Strongly agree \\
& $\bar{x}=4.75$ & $\bar{x}=4.98$ & $\bar{x}=4.93$ \\
\hline
\end{tabular}

Another positive outcome which may speak to the effectiveness of the educator and education method may be seen as three of the five pilot participants requested to watch the optional fourth module to learn how to create their own low-technology communication 
board. None of the pilot participants chose to upload an example of a created board, however, they were given access to the template for future use.

Additional open-ended questions were posed at the end of each module post-test to determine how participants felt their behavior would change, benefits of the CBLs, barriers to using this information, and facilitators. Four of the five pilot participants highlighted their new awareness of and plan to use wait time in their clinical work following module 1. All participants felt a benefit would be improved communication with their patients. Two of the participants also mentioned the likely benefit of patients feeling more cared for and listened to as they provide more time to communicate. A variety of barriers were mentioned regarding the module 1 content including, cognition for some patients, parents jumping in and speaking for the patient in the midst of wait time, limited resources, and fast paced work culture that does not allow them to take extra time. One participant reported no barriers to using these strategies as they felt their facility was very supportive of and focused on improving communication. Pilot participants reported management support was a facilitator in four of the five facilities and one reported they felt that proper training and tools were facilitators for their implementation of this information.

All five participants reported they had learned new information that they plan to use in their clinical work including simple sign language, tips for choice making, and communication boards. Regarding the benefits of the information learned through module 2, all participants reported they felt they could better support communication for their patients after learning this information. One participant stated, "The more communication techniques I know and understand the better outcome for my patient." Access to lowtechnology AAC was a primary barrier communicated after this education module $(n=3)$ as well as cognition. One participant reported no barriers to using information covered in this module in her clinical work. Management was again mentioned as the primary facilitator with three participants also mentioning the importance of working with rehabilitation staff and feeling supported by their medical team as a facilitator in their clinical environment.

Four of the five participants reported aspects of their clinical work that would change following their participation in the third education module. Reported changes included finding and using more communication boards, not giving up if communication does not happen immediately, and more tools to attempt communication efforts with patients. All participants reported that they had benefited from the information with most ( $\mathrm{n}=$ 4) reporting they felt they could be more effective at communicating with their patients because of this information with less anxiety and frustration. One participant reported she felt she now knew the names and terms of what she already does in her clinical work. Three of the five pilot participants reported access being the primary barrier to using module 3 information and two additional participants reported they had no barriers. All five participants reported that feeling supported by management, medical team, and rehabilitation staff were facilitators to implementing this information clinically.

No participants had additional questions at the end of modules 1 and 2 . Two questions were posed at the end of module 3 during this pilot: "I feel like the scale of 
comfortability was weird, because we don't use these words or terms, but may already be practicing them. Saying utilizing a communication board versus patient scanning (I had never heard of before), but is what I do," and "How can staff get these boards on their floors?" The scale the participant referred to above was a validated scale (L-SES) therefore minimal wording changes were made to maintain the validation of the scale (Kang et al., 2019). The second question was addressed through the provision of handouts, physical communication boards for those interested in receiving them, and a digital handout with links to freely available online communication boards for nurses to access as needed.

Participants were also asked to provide their top five vocabulary that they feel would be most helpful to have on a communication board or for choice making for their patients. Responses included: bathroom/potty $(\mathrm{n}=5)$, pain/ouchy/hurt $(\mathrm{n}=4), \mathrm{mom} / \mathrm{dad} /$ family ( $\mathrm{n}$ $=2)$, TV / movie $(\mathrm{n}=2)$, food $(\mathrm{n}=2)$, stop $(\mathrm{n}=1)$, happy $(\mathrm{n}=1)$, belly $(\mathrm{n}=1)$, position/move $(\mathrm{n}=1)$, drink $(\mathrm{n}=1)$, cold $(\mathrm{n}=1)$, medicine $(\mathrm{n}=1)$, bath / shower $(\mathrm{n}=1)$, bed change $(\mathrm{n}=1)$. Pilot participants reported that they interact with patients weekly who would benefit from low-tech AAC: 1-2 ( $n=2), 3-4(n=1), 7-8(n=1), 8+(n=1)$. Three of the five participants reported they have access to low-tech AAC in their facility and the same three participants reported they could access low-technology AAC during their next shift. Participants were asked who usually provides low-tech AAC supports in their facility and they reported nursing $(n=1)$ and SLPs $(n=3)$. They also reported referring to speech language pathology to varying degrees $(<1$ time per month $(n=3), 3-4$ times per month $(n=1),>1$ time per week $(\mathrm{n}=1)$ ). Three participants requested additional information on AAC.

Following this successful pilot phase with participants providing feedback, no additions, subtractions, or changes were recommended or indicated. A question was added to the screening portion of the research requesting a professional email address to verify participants' eligibility to participate. Once these changes were finalized and approved, recruitment for full scale data collection began on 11/9/20.

\subsubsection{Trialing of New CBLs on AAC}

After the preliminary pilot phase of the CBLs were completed $(n=5)$, information for participation in the CBLs was widely distributed. Nurses and nursing students were recruited via social media, posted flyers, and via word of mouth to potential participants in this implementation study. All participants were provided with an online screener to ensure they met the inclusion criteria (18 years of age or older, a pediatric nurse or nursing student) and were willing to be contacted via email to participate in this research. All participants who met the inclusion criteria and were interested in participating were given the same preand post-assessments for each of the three online education modules.

All data was anonymously collected through Qualtrics, an online survey generator and data collection tool. Data was collected from November 9, 2020 - February 15, 2021 with 44 nursing students and 25 pediatric nurses across the United States. A total of 131 individuals completed the screener and were provided with an anonymous link to the online CBLs. Eighty-three potential participants started the CBLs (63.36\% began the modules) 
and 70 completed all three modules with a 53.44\% completion rate. One participant selfidentified as a physician's assistant and therefore was not included in the data analysis.

Following data collection, data analysis included assessment of the four primary outcomes (knowledge, self-efficacy using AAC, relevance of the content, and perceived effectiveness of the education) via within subjects ANOVAs or t-tests using SPSS (IBM Corp., 2017). Within-subjects ANOVAs assessed the data for knowledge and self-efficacy using AAC for pre- and post-assessment measures for each module and across all three modules. Additional independent samples t-tests were completed if further investigation of the data was indicated. Between subjects ANOVAs compared data based on current classification (i.e., pediatric nurse vs nursing student). L-SES, relevance, and effectiveness scales were explored through descriptive statistics, following calculation of reliability (Cronbach's alpha) and validity data.

\subsubsection{Participants}

Participants were recruited to participate in this research using social media, posted flyers, and personal contacts. A variety of universities with nursing programs, children's hospitals, pediatric nurse and student associations, and social media groups were contacted with information about this research. Seventy participants completed all three education modules; one participant self-identified as a physician's assistant thus their data was not being including in the final analysis. The sixty-nine participants self-identified as either a nursing student $(n=44)$ or pediatric nurse $(n=25$; see Table 3.4).

Nurses are often continuing their education, thus there were five participants who self-identified as a working pediatric nurse and nursing student pursuing an advanced degree, thus they were included in the pediatric nurse group since they reported continuing to work while in school.

Nursing students reported pursuing the following degrees: BSN ( $\mathrm{n}=40), \operatorname{ABSN}(\mathrm{n}=$ 3), $\operatorname{ADN}(n=1)$. The five pediatric nurses pursuing advanced degrees were obtaining MSN $(\mathrm{n}=3), \mathrm{BSN}(\mathrm{n}=1)$, and DNP $(\mathrm{n}=1)$. A variety of nursing programs were represented including The University of Tennessee Knoxville $(n=22)$, Augusta University $(n=8)$, The University of Kansas $(n=6)$, East Tennessee State University $(n=2)$, South College $(n=$ $2)$, The University of Memphis $(n=2)$, Carolina College of Health Sciences $(n=1)$, The University of Oklahoma $(\mathrm{n}=1)$, and The University of South Alabama $(\mathrm{n}=1)$. Information was not gathered regarding pediatric nurse employment.

Most participants were from the Southeast, however, an additional approximately $19 \%$ were from the Midwest. The only region not represented was the West, however, there were four missing responses for region. Age, gender, and racial demographic information was not collected in this research. 
Table 3.4: Demographic Information.

\begin{tabular}{|c|c|c|}
\hline Demographics & Frequency & Percent \\
\hline Pediatric Nurse & 25 & 36.20 \\
\hline Nursing Student & 44 & 63.80 \\
\hline \multicolumn{3}{|l|}{ Practicing Healthcare Provider } \\
\hline Yes & 34 & 49.30 \\
\hline No & 35 & 50.70 \\
\hline \multicolumn{3}{|l|}{ Role } \\
\hline Nurse & 24 & 72.20 \\
\hline CNA & 7 & 21.20 \\
\hline Nurse Manager & 1 & 3.05 \\
\hline Nurse Technician & 1 & 3.05 \\
\hline \multicolumn{3}{|l|}{ Department } \\
\hline Cardiology & 1 & 3.13 \\
\hline Emergency Department & 3 & 9.09 \\
\hline Geriatrics & 2 & 6.26 \\
\hline GI/Endocrine & 1 & 3.13 \\
\hline Home Health & 1 & 3.13 \\
\hline Medical-Surgical & 3 & 9.09 \\
\hline Medical-Behavioral & 1 & 3.13 \\
\hline Medical-ICU & 1 & 3.13 \\
\hline Multiple/Floater & 3 & 9.09 \\
\hline NICU & 3 & 9.09 \\
\hline Oncology & 1 & 3.13 \\
\hline Operating Room & 1 & 3.13 \\
\hline Outpatient & 4 & 12.12 \\
\hline Pediatrics & 2 & 6.26 \\
\hline PICU & 2 & 6.26 \\
\hline Public Health & 1 & 3.13 \\
\hline Renal & 1 & 3.13 \\
\hline Trauma & 1 & 3.13 \\
\hline \multicolumn{3}{|l|}{ Shift } \\
\hline Day & 26 & 78.79 \\
\hline Night & 6 & 18.18 \\
\hline Rotating Day/Night & 1 & 3.13 \\
\hline \multicolumn{3}{|l|}{ Employment Status } \\
\hline Full-Time & 20 & 60.61 \\
\hline Part-Time & 2 & 6.06 \\
\hline PRN & 11 & 33.33 \\
\hline \multicolumn{3}{|l|}{ Geographic Region } \\
\hline Northeast & 2 & 2.90 \\
\hline Southeast & 47 & 68.16 \\
\hline Midwest & 13 & 18.84 \\
\hline Southwest & 3 & 4.35 \\
\hline West & 0 & 0.00 \\
\hline
\end{tabular}




\subsubsection{Funding and Incentives}

This research was funded by a Diversity and Inclusion Mini-Grant through the University of Tennessee Health Science Center Office of Equity and Diversity for \$1,250.00. The grant funds were provided to print and laminate low-technology communication boards and to provide a small monetary incentive for participants.

Participants who took part in the piloting and trialing phases of this research were given the opportunity to receive, via mail, double-sided color, laminated low-technology communication boards to use in their clinical work. The Patient Provider Communication Network and Boston Children's Hospital have freely available low-technology AAC boards online which they allowed us to print and provide to participants for this research, with appropriate citation and credit given. In addition, they were given, via email, a digital \$10 Amazon gift card as a thank you for their participation in the study. 


\section{Chapter 4}

\section{Results}

\subsection{Qualitative Results}

RI-1. What AAC and communication content do pediatric nurses and nursing students find relevant to their clinical work?

Qualitative data collection through interviews and focus groups were analyzed as discussed in the previous chapter to characterize the thoughts and experiences of these populations. The results provided shared themes related to educational and clinical experiences and preferences regarding alternative communication.

The primary overarching themes identified in this research included communication, clinical experience, and educational experience, which are defined in Table 4.1. These overarching themes included a variety of sub-themes which varied slightly between data collected from pediatric nurses and nursing students, thus the results will be discussed separately.

\subsubsection{Pediatric Nurse Data - Interviews and Focus Groups}

Ten pediatric nurse participants from Tennessee $(n=8)$ and California $(n=2)$ participated in a focus group or interview discussion indicating a variety of clinical and educational experiences regarding communication and AAC.

\section{Communication}

Communication was a main topic of conversation with all nurse participants, however, various areas related to pertinent terminology, importance, and types of communication were also highlighted by participants. The pediatric nurses who participated in this qualitative portion of the research understood and felt the importance of communication in their jobs. They often spoke of situations where communication was particularly difficult whether due to content matter, emotional state of the communication partner(s), or medical status of the patient (ex., medically fragile, intubated, congenital disability, etc.). Pediatric nurses communicated their understanding, based on clinical experience, of the major role that communication plays in their daily patient interactions. 
Table 4.1: Qualitative Themes and Definitions.

\begin{tabular}{|c|c|}
\hline Themes & Definitions \\
\hline 1. Communication & $\begin{array}{l}\text { The variety of information exchanges, including verbal and non-verbal } \\
\text { means, through an interchange of information, thoughts, feelings, } \\
\text { desires, wishes, and preferences required for mutual understanding in } \\
\text { healthcare provision. }\end{array}$ \\
\hline 1.a. Terminology & $\begin{array}{l}\text { Definitions of terms used in communication and alternative } \\
\text { communication methods. Terms include AAC, Therapeutic } \\
\text { Communication, Communication, Effective Communication, Complex } \\
\text { Communication Needs, Patient-Provider Communication, etc. }\end{array}$ \\
\hline $\begin{array}{l}\text { 1.b. Importance of } \\
\text { Communication }\end{array}$ & $\begin{array}{l}\text { Described and perceived importance of communication in clinical } \\
\text { settings. (ONLY found with pediatric nurse qualitative data) }\end{array}$ \\
\hline $\begin{array}{l}\text { 1.c. Types of } \\
\text { Communication } \\
\text { Supports \& Strategies }\end{array}$ & $\begin{array}{l}\text { Types and strategies of communication mentioned - they may be used } \\
\text { or simply known of and include smaller sub-themes of no-tech, low- } \\
\text { tech, mid-tech, and high-tech AAC. }\end{array}$ \\
\hline 1.c.i. No-tech & $\begin{array}{l}\text { No-technology communication strategies named or described in } \\
\text { interviews and focus group discussions (ex., sign language, body } \\
\text { language, facial expressions). }\end{array}$ \\
\hline 1.c.ii. Low-tech & $\begin{array}{l}\text { Low-technology AAC strategies and supports described or named in } \\
\text { interview and focus group discussions (ex., communication boards). }\end{array}$ \\
\hline \multirow[t]{2}{*}{ 1.c.iii. Mid-tech } & $\begin{array}{l}\text { Mid-technology AAC strategies and supports described or named } \\
\text { during interview and focus group discussions (ex., talking box, }\end{array}$ \\
\hline & $\begin{array}{l}\text { GoTalk20+, Step-by-Step). These includes digitized speech and an } \\
\text { outside power source such as batteries. }\end{array}$ \\
\hline 1.c.iv. High-tech & $\begin{array}{l}\text { High-technology AAC strategies and supports discussed during } \\
\text { interview and focus group discussions (ex., tablet applications, eye-gaze } \\
\text { devices). These include dynamic displays, digitized and synthesized } \\
\text { speech, and a power source. }\end{array}$ \\
\hline 2. Clinical Experience & $\begin{array}{l}\text { Experience in clinical settings which may include internships, clinical } \\
\text { rotations, and paid clinical work for nursing students and practicing } \\
\text { pediatric nurses. }\end{array}$ \\
\hline \multicolumn{2}{|l|}{ Experience with AAC } \\
\hline $\begin{array}{l}\text { 2.a.i. Populations } \\
\text { that May Benefit } \\
\text { from AAC }\end{array}$ & $\begin{array}{l}\text { Populations who might benefit from alternative communication } \\
\text { supports and strategies as evidenced by clinical experience and } \\
\text { nurse/student observation of communication support opportunities. }\end{array}$ \\
\hline $\begin{array}{l}\text { 2.b. Barriers \& } \\
\text { Facilitators }\end{array}$ & $\begin{array}{l}\text { Barriers and Facilitators to using AAC as evidenced through clinical } \\
\text { experiences. }\end{array}$ \\
\hline 3. Education & $\begin{array}{l}\text { Educational experiences of participants which may include formal } \\
\text { education, clinical experiences, or continuing education. Additional } \\
\text { discussion of educational needs and patient education are discussed. }\end{array}$ \\
\hline $\begin{array}{l}\text { 3.a. Educational } \\
\text { Experience }\end{array}$ & $\begin{array}{l}\text { Educational experience regarding communication and AAC as a student } \\
\text { or practicing nurse. }\end{array}$ \\
\hline 3.b. Educational Needs & $\begin{array}{l}\text { Educational needs regarding communication and AAC felt by } \\
\text { participants. }\end{array}$ \\
\hline 3.c. Patient Education & $\begin{array}{l}\text { Patient education provided and ways that nurses adapt that information } \\
\text { to ensure effective communication. (ONLY found with pediatric nurse } \\
\text { qualitative data) }\end{array}$ \\
\hline
\end{tabular}


Among desired areas of improvements regarding communication, was communicating information to NICU families consistently, learning sign language and Spanish, improving their ability and knowledge about technology, and being able to speak to patients and families in an appropriately health literate manner. They identified the most common communication breakdowns, in their experience, to be between healthcare providers, providers and families, and due to language differences.

Terminology. Communication was defined by one nurse as, "verbalizing between two or more people to solve a problem;" however, upon further discussion, nurse participants were able to provide a variety of examples of communication methods outside of and in addition to verbalizing, including the use of technology such as telephones or Zoom, facial expressions, body language, text through texting on a cell phone or emailing, sign language, pen and paper, and a white board. Other definitions by nurses included the importance of the two-way exchange of information, expression of thoughts by both parties in the communication exchange, and listening and understanding.

Definitions provided for effective communication did not differ drastically from initial definitions of communication in that there was a focus on the back and forth exchange of information with mutual understanding of the content. This is ideal, as we hope that all communication is effective, or else it begs to question whether it is communication at all. They reported a slightly different goal between communication and effective communication with the latter focusing on building trust with patients and caregivers, much like later discussions of therapeutic communication.

One pediatric nurse reported she had not heard the term 'communication vulnerable' or the descriptive term of 'complex communication needs;' however she hastened a guess that it referred to someone with "difficulties communicating." Another pediatric nurse with a family member with Autism Spectrum Disorder (ASD) reported that her family member would be an example of someone who was communication vulnerable.

Two additional terms discussed during the interviews and focus groups were receptive and expressive communication. These terms were not familiar to the pediatric nurse participants; however, they appeared transparent in nature with most nurses able to guess the meaning of each of them. Expressive communication appeared a more familiar term, with participants more confidently describing what it would entail. Two of the ten participants reported good familiarity with both terms, one of them stating they had learned about them in a leadership course that had specific instruction on communication techniques.

Two of the ten participants were familiar with the term AAC and could describe the gist of the acronym with one able to expand it appropriately; however, most participants were completely unfamiliar with the acronym. The PI provided each participant and group with a definition of the acronym and following this description a few additional participants were able to summarize what they understood the acronym to mean. One nurse participant reported she had heard the term alternative communication and was familiar with examples of it; however, had never heard the acronym AAC before. 
Pediatric nurses had more exposure and experience with AAC compared to the nurse student data described below, albeit quite varied based on unit, experience, and possibly geographic location or facility. Most participants, when given descriptions or visible examples of different types of AAC, were familiar with low-technology and hightechnology AAC options. Many reported using low-tech methods such as pen and paper out of the need to provide additional information or education to patients and families. Participants were familiar with or had seen a few high-technology AAC such as tablets with applications and a handful of communication devices from particular companies.

Types of communication supports and strategies. In addition to the previously mentioned ways that we communicate, pediatric nurses were quite familiar with translation services, Cyracom, and supports such as UbiDuo and Deaf Connect for individuals who are deaf or hard of hearing. Also, as mentioned below in the Barriers and Facilitators section, nursing participants reported knowing of a variety of communication supports (ex., tablets with applications, letter boards, paper and pen) that were managed by their Speech Language Pathology or Child Life departments, but were unable to consistently access them or keep them at the bedside for patients in all situations.

An additional method of communication support mentioned was the use of biofeedback in a urology clinic and images from procedures or tests. The nurses mentioned the ability for a child to watch and see what is happening on the ultrasound to better understand their condition or a procedure that might be coming up. There was also the mention of using the TVs in rooms and tablets to provide education or information to patients and families; it was highlighted as an avenue of sharing information with patients but as a single-sided communication effort.

No-technology AAC examples that nurses were able to provide included snapping fingers, blinking, and squeezing a hand for yes and not squeezing for no. Low-technology strategies that they reported using included pen and paper to draw or write out information such as drawing a picture of a heart defect, a dry erase board and marker, communication boards, and a puppet or stuffed animal to talk through. Mid-technology AAC examples provided by participants included a box that had buttons on each side where a picture or word could be slid inside a pouch and the button could be programmed with digitized speech to match the images or words. Another pediatric nurse had experienced a speech generating single message communication device like a Step-by-Step ${ }^{T M}$ in her personal life with a family member but not in her clinical experience. High-technology AAC was most familiar to participants through the prevalence of tablets with communication applications.

The importance of communication. Pediatric nurse participants specifically stated the perceived importance of communication in their clinical role with one nurse stating, "I'm passionate about making sure that people understand when you're communicating." Another nurse reported using communication strategies, "every shift, multiple times a shift." 
One nurse reported she would talk to her supervisor regarding this research as she felt that the content was worth disseminating across their facility to assist their nurses. Additionally a nurse who was preparing for a job change, reported she felt information on AAC and communication strategies could be really helpful as she transitioned into her new role. She reported, "we've learned a lot about how to communicate with patients verbally and using technology because as a nurse practitioner that is one of the things that you do all the time, communicate."

\section{Clinical experience}

The pediatric nurses who participated in the qualitative data collection, were from a variety of units, backgrounds, and experiences. Most were full-time nurses ( $n=9)$, with one working pro re nata (PRN) as a floater but often in the NICU. The full-time nurses were in pediatric emergency department (ED), PICU, NICU, Cardiovascular Intensive Care Unit (CVICU) - pediatrics, and out-patient pediatric urology. Degrees ranged from License Practical Nurse (LPN, $n=2$ ), to Registered Nurse (RN, $n=5)$, to Nurse Practitioner (NP, $\mathrm{n}=2$ ), and Physician's Assistant (PA; $\mathrm{n}=1$ ). In addition to their current employment, participants had experience in ED, PICU, Adult nursing, Oncology, Med-surg, Well Baby Nursery, and labor and delivery. Clinical nursing experience ranged from nine months to eleven years. Most of the nursing participants worked day shift (8 am - 5 pm or 7 am $7 \mathrm{pm}$ ), however, there was one PRN who worked mostly weekends and some weekdays when needed, and an evening shift nurse who worked 3 - 11 pm.

Clinical experience with $A A C$. All pediatric nurse participants were able to think of situations in which alternative communication methods were used or would have been useful. Many reported clinical experiences that were void of communication supports. Nurses reported no access to communication help in the ED, communication being directed solely at parents if the patient is unable to communicate effectively. The particular difficulty with patients and families who are non-native English speakers was reported often, with one nurse stating she draws a lot of pictures with those families to explain things.

Pediatric nurses reported that they often use the alternative communication methods discussed during this research such as pen and paper, the language line, and no-technology strategies like eye blinks or hand squeezes for yes/no responses. When queried, nursing participants reported the need for AAC in their clinical experience ranging from once or twice a month to every day, which appeared to be situation, unit, patient, and nurse specific. Six of the ten nurses reported they had never seen or used anything outside of the most basic forms of alternative communication (ex., pain scale, pen and paper, white board), including never seeing a communication board or tablet with an application. Those nurses were most familiar with language support tools like the language line and pen and paper type communication supports that were made in the moment. Two pediatric nurses reported seeing tablets that patients brought in for communication in the emergency department. One nurse was familiar with mid-technology AAC from her clinical work and another had seen and used it with a family member but not in her clinical work. 
Populations that may benefit from AAC. A variety of patient populations were mentioned who were considered communication vulnerable including those with hearing impairment, visual impairment, speech delay, lower education level, ASD, speech problems, non-native English speakers, syndromes and cardiac issues, Rett's, seizure disorders, Down Syndrome (DS), CHARGE, Marfan's, Treacher Collins, spinal surgeries, post-tonsil and adenoidectomy (T\&A), traumatic brain injuries (TBI), brain tumors, and patients who are intubated but not sedated. One travel nurse who worked in an ICU reported she felt AAC could benefit not just certain diagnoses but really all children from toddler ages and up to support communication and combat fear and shyness among other things.

Nurse participants reported a familiarity with language services such as Cyracom; however, they also mentioned the benefit of having a low-technology AAC board available to them for a simple schedule of what kids can expect with certain procedures or vaccinations using pictures and simple words. One nurse made this recommendation in particular to be used with children with ASD who may struggle to understand what is going on as well as have difficulty expressing what they are thinking or feeling, let alone be able to ask questions about what is happening.

In addition, pediatric nurses provided a general idea of how often they felt they would use AAC and communication supports if they were available. Some settings, such as the NICU, did not feel there was a high priority of AAC and communication supports for use with patients but that they could be helpful in interacting with and teaching parents of newborns. One nurse reported she uses AAC strategies every week, every shift, multiple times a shift, even being in the NICU. One nurse reported she sees anywhere from one to four or more patients per day who could benefit from AAC supports. Another pediatric nurse working in urology stated, "If I had one of those [referencing a communication board specific to their practice] I would use it a lot. I would try to incorporate it with every patient with autism that I have, even the ones that are verbal." Another nurse on a pediatric cardiovascular ICU reported she felt there was a place for AAC and communication supports with $30-40 \%$ of her patients for support during post-surgery intubation, though she reported the use of AAC would be nurse and patient dependent.

Barriers and facilitators. A variety of barriers and facilitators regarding the use of $\mathrm{AAC}$ and communication strategies were identified by pediatric nurse participants both within their facilities, with medical facility culture, and personal barriers and facilitators. Access, expectations, time, language differences, lack of knowledge, and staffing were commonly mentioned barriers to the use of AAC. Additional barriers mentioned included fear of asking questions and looking less competent as a reason that nurses may not ask where to find or how to use certain communication supports. Medical status was a barrier mentioned by multiple nurses $(n=2)$. One PICU nurse reported that AAC can be a distraction or something that a child may perseverate on. Some children will press the buttons over and over to hear the message, not because they actually need to communicate something but out of boredom or because they find it humorous. This may lead to nurses taking that communication support away or disabling it which may assist 
with the immediate 'alarm' fatigue, but would have the long-term ramification of removing communication opportunities for that child.

Time was discussed by multiple nurses as a barrier. One nurse mentioned that she may go through the trouble to get an interpreter to come up to the floor but by the time they are able to get there, the patient and family may already be gone. "It needs to be like readily available because I mean sometimes they communicate something quickly, I mean you're not able to, you're just not able to." An additional barrier related to time is the need for many AAC techniques, especially mid- and high-technology AAC, to be charged or to have working batteries.

Additional concerns were voiced regarding that fact that AAC can be intimidating to someone who has never seen or used it before. They may be afraid to try for fear of looking incompetent or because it takes additional time, as mentioned above. The cost related to AAC supports is also a barrier for many healthcare facilities who often have limited budgets. Infection control is a hospital-level concern for AAC supports; however, most purchased AAC is considered durable medical equipment and can be cleaned with hospital-grade cleaners. In addition to infection control, is the concern for where these supports will be housed and who will maintain them.

An additional barrier, as mentioned previously, was that pediatric nurses were aware of a few disciplines who had tablets, but they were unsure whether they included communication software and they were not readily available if those disciplines were not present. Child Life was one discipline, though it was commonly understood that their tablets were available strictly for distraction, not for communication support. The other mentioned discipline was Speech Language Pathology and one nurse had seen an SLP use a tablet in therapy but knew that they were not available to be left in patient's rooms. One nurse reported they have phones dedicated to communication between healthcare providers (phone calls and texts) but that you are not able to download apps onto those phones, nor could they be left in a patient's room as a more long-term communication support.

In addition to the numerous barriers, a few facilitators were identified including the multidisciplinary team atmosphere at many hospitals, currently used technology in rooms for education or distraction that may be adapted for communication, interpretive services language lines, Cyracom, Deaf Connect, UbiDuo, and the ability to tailor low-technology communication boards for patients and units. Time was also mentioned as a potential facilitator, if AAC methods that are quick and easy can be taught and implemented.

\section{Education}

Educational experience. The nurse participants came from a variety of locations and educational backgrounds, with most having pursued their nursing degree in undergraduate level work and some who had additional higher level degrees such as pediatric 
nurse practitioner. One nurse discussed feeling rather prepared for her clinical work following her undergraduate schooling and clinical rotations. She said, "I felt like we were pretty prepared, they did a really good job with diversity and making sure that we understood different cultures and how they respond to non-verbal and verbal interactions and they also had simulations....and they really tried to make sure that we understood the different people who are going to be at the hospital, that you're going to be working with...so I would say yes, I felt very prepared."

Most of the nurse participants discussed their preferences and experience regarding continuing education rather than undergraduate or graduate level coursework. Participants reported a few companies that provide continuing education for nurses as well as some education that is conducted in-house at their facilities. They reported that the education varied in length from 15-30 minutes up to 3-4 hour long courses for more advanced content. They stated that most education modules, if online, would allow you to start, stop, and pick them up later.

Educational needs. Overall, nurse participants reported that education on AAC and communication supports and strategies would be beneficial to pediatric nurses with one of two NICU nurses reporting it could even benefit their unit to improve healthcare provider and family interactions. Multiple nurses reported that having this type of information available for new nurses at on-boarding training would be beneficial. Two nurses were recent graduates of nursing programs (working less than 2 years) and they both reported they had not learned any of this information in school but that it would be helpful for their clinical practice.

Nurses repeatedly highlighted the importance of and limited amount of time available to their discipline, thus they requested that education modules remain brief and focused with the ability to start and stop them. They requested two to four modules lasting around 15-30 minutes each rather than a single 60-120 minutes long module.

Nurse participants reported a preference for access to low-tech communication boards including picture boards $(\mathrm{n}=4)$, mid-technology AAC $(\mathrm{n}=1)$, and high-technology AAC $(n=2)$, with three nurse participants not voicing a preference. In addition, nurses requested more information on a variety of topics regarding the attainment and more information about AAC: the process of getting someone a communication support $(n=1)$, what options are available $(n=3)$, who uses AAC $(n=1)$, what strategies are helpful and research-based $(n=2)$, whether AAC is durable medical equipment $(n=1)$, and applicable resources regarding insurance coverage $(n=1)$. Additional questions participants had included who to refer to $(\mathrm{n}=1)$, and whether Child Life could support communication efforts $(n=1)$. Some participants wanted to know how to access and use high-technology AAC options, what to do if no-technology/low-technology efforts in place were not working for a patient, and more information on tablets and apps.

Patient education. Pediatric nurse participants often mentioned using communication supports and strategies during discharge and patient/family education scenarios. Most 
nurses mentioned that patients and families were provided with a lot of printed materials and handouts at discharge to ensure carry over of information. One difficulty reported with educating patients and families was the emotional barriers or difficulties many families and patients are enduring during hospitalization including denial and grief regarding a diagnosis or prognosis.

A variety of technology was discussed regarding patient education including education modules on the TVs in each patient room, videos for teaching information like $\mathrm{CPR}$, an application on a provided phone to teach patient families about heart anatomy and function, and use of biofeedback tools and imaging studies like ultrasound and x-rays. Another teaching tool that many nurses mentioned was the use of demonstrations, teach back strategies, or a process called learn one, do one, teach one. In that technique patients are taught a skill or concept (ex., changing an ostomy bag), then provided the opportunity to 'do one', then asked to 'teach one' to another family member. Additional discussion on communication supports used during patient education included the importance of health literacy and ensuring all healthcare providers are using language and terminology that is easily understood. Multiple nurses reported drawing or using picture supports when explaining information to families and patients.

\subsubsection{Nursing Student Data - Interviews and Focus Groups}

\section{Communication}

Nursing student participants reported a solid foundation regarding their understanding of communication, specifically therapeutic communication, but also voiced a desire to know more. Participants reported a desire to listen more and ask better questions of patients. They reported making attempts at being sensitive to cultural and language barriers and to be sure they are seen and heard by patients who may have a vision or hearing deficit. One nursing student mentioned the importance of communicating not only with pediatric patients but also their parents as this aids in keeping the patient at ease.

Terminology. Nursing students consistently defined communication as including a two-way exchange of information using words, body language, and non-verbals. One nursing student defined it as, "how they express how they feel; giving and receiving critical information." Another student described communication as, "a message or idea being transferred to someone else, so you can do that verbally, and then there's different aspects of that or non-verbally and we use different methods and means of accomplishing that." Patient-provider communication was a new term for most participants and often brought to mind doctor-patient communication, not considering themselves as part of the termed 'provider'.

They identified aspects of effective communication such as listening, asking openended questions, communicating clearly, reading non-verbal cues that may communicate more or differently than verbal speech, and making sure patients and families understood what was going on. Some of the characteristics described overlap with participants' 
definitions of therapeutic communication which included open-ended questions, getting information from patients in a non-judgmental way, and trying to be a kind nurse. All nursing student participants discussed therapeutic communication and reported receiving education on this type of communication. One nursing student said they hoped, as a nurse, to be "the support system for the patient." Another student differentiated therapeutic communication from basic or effective communication by stating, "it has a different goal," which was described as more focused on building rapport with patients and ensuring they feel comfortable communicating personal information.

Nursing students, like pediatric nurse participants, were unfamiliar with the term communication vulnerable. The populations that nursing students identified as possibly being communication vulnerable included hearing and visual deficits, education level, language barrier or difference, abuse (ex., verbal, physical, sexual), individuals with low confidence (ex. individuals who stutter), and individuals post-stroke who cannot communicate due to aphasia.

One of the nursing students who participated had heard the term expressive communication but receptive and expressive communication were largely new terms for most nursing students. They did feel that these were good terms to know and helpful when thinking more deeply about the communication exchange. As mentioned above, therapeutic communication was mentioned often by nursing students, but patient-provider communication proved to be a new term or a term that encapsulates a broader group of providers than just doctors, as mentioned above.

The acronym AAC was a brand new term for participants. Even when this acronym was expanded and explained, only one nursing student (practicing as an LPN) could provide an example of AAC - the smiley face pain scale. When provided with visual or descriptive examples of no-tech, low-tech, mid-tech, and high-tech AAC supports and strategies, most nursing students reported not having seen or learned about these tools. One nursing student had seen a low-tech board used in another country with a patient and another nursing student had seen a patient use a tablet with a communication application at a rehabilitation facility where they worked. Overall, these were new vocabulary, tools, and supports to the nursing students who took part in this qualitative research.

Types of communication supports and strategies. The nursing students provided a variety of examples of types of communication supports or strategies such as language translation, gestures, facial expression, body language, and written communication including emails and text. None of the nursing students were aware of the term AAC, as mentioned above. Once no and low-technology, mid-technology, and high-technology AAC were described, most participants were able to, with more confidence, state that they had never seen these types of communication supports in their education or clinical experiences. Participants were exposed to visuals or descriptions of a variety of AAC and communication supports during the qualitative data collection discussion. Most nursing students reported awareness of the pain scale and reported using some no-technology strategies such as gestures, facial expressions, and recasting. 


\section{Clinical experience}

All of the nursing students who participated in a focus group or interview were currently in clinical placements with a variety already completed and at least one or more left to complete in each of their programs. Clinical rotations discussed included psychiatry in rehabilitation facilities, clinics, and mobile health/mobile meals, medical-surgical, mental health, obstetrics, labor and delivery, gynecology, pediatrics, population health, primary care, and adult health. Students described two to four year nursing programs with 10-11 clinical rotations completed throughout the entirety of each program. Clinical rotations included two to three 12-hour shifts, 10 days per placement, and a 12 week practicum indicating variety in the length of time and way that clinical rotations are executed across nursing programs. The students who participated in this portion of the research were interested in working in a variety of areas including pediatrics, geriatrics, psychiatry, mental health, and undecided.

Clinical experience with AAC. Participants $(n=3)$ reported interacting with patients with CCN during their clinical rotations thus far with the populations mentioned including dementia, Parkinson's Disease, stroke, cerebral palsy, and renal failure. Communication strategies that they reported using in their clinical rotations included body language, speaking clearly and loudly, tone, interpreters, therapeutic communication, posted signs in rooms, facial expressions, and Pocket Talkers. One nursing student working in geriatrics told a story of creating a communication board for a non-native English speaking patient: "We had to make her a board in her native language which is Arabic...we asked her daughter to write out what it needed to be and we stuck it on there." Another nursing student reported seeing a low-tech communication board twice while in a hospital clinical rotation in the Philippines. Two of the five nursing students had experience seeing low-tech AAC, with one of them having used a low-tech AAC support. All nursing students were familiar with the pain scale in clinical work.

When discussing self-efficacy, someone's perceived comfort or confidence using an AAC method or tool, all nursing students in this research reported feeling comfortable using simple AAC supports like a pen and paper or marker and whiteboard. Two nursing students reported feeling comfortable, or at least willing to try, using a communication app on a tablet.

Populations that may benefit from AAC. Nursing students recalled examples of patients from their clinical rotations that may have benefited from AAC supports. One nursing student discussed a non-native English speaking patient in the hospital setting who did not use the call light correctly when on the bedside commode: "I've heard this twice actually where someone was on the bedside commode and you know 'don't get up' and they can't understand you, so it's like 'do you understand-eh?', like someone said that." This student was aware that what they witnessed was ineffective communication between staff and the patient and the student recognized that this was not only culturally insensitive but that it could have been an opportunity to use AAC supports to teach and support communication for patients and staff. One nursing student discussed the benefit of 
photos with low-technology communication boards for patients who are having trouble communicating, highlighting the universality of pictures that can cross language barriers.

All of the nursing students were able to provide examples of patients or patient populations who might benefit from the use of AAC. The primary populations they discussed included individuals who have had a stroke, individuals with dementia, non-native English speakers, the elderly, and pediatrics. In addition to identifying these populations, they also discussed the potential benefit of having communication supports in every hospital room. Participants also discussed that, as there are certain patient populations who are more likely to need AAC support, the likelihood of using AAC regularly would be setting or unit specific. Two of the five nursing students reported they felt sure they would use AAC at least once a day in some capacity if it were available. One nursing student stated, "that feels like something that should be in every supply closet," in regards to mid-tech devices such as a Step-by-Step ${ }^{T M}$ or GoTalk $20^{T M}$.

Barriers and facilitators. Nursing students provided a variety of barriers and facilitators to the use of AAC in clinical settings. Among the primary barriers mentioned was access and knowledge of use, time, nurse buy-in to learn and use AAC, and patient cognition. Lack of access to AAC supports was mentioned throughout the interviews and focus groups, as most participants had never seen AAC supports in their clinical rotations thus far. This is a primary concern, as if there is no access to AAC supports, it is impossible for them to be used or for training/education to be relevant. In addition, if someone does not know what communication supports are available then they cannot use those supports. Participants also reported time, workload, and staffing issues as barriers that result in nurses doing the bare minimum to keep all patients cared for: "It [student describing a clinical rotation when a nurse called out of work] was kind of panicky. And so everybody was basically just trying to do their med pass and get everybody taken care of and treat trauma that needs to be treated." From the student perspective, a lack of motivation from more seasoned nurses was also mentioned as a barrier to AAC use. One student reported experiencing an attitude from a preceptor of, "that's not the way we have been doing it for 30 years and you want me to do that? No."

Barriers with AAC included the time that it takes to keep AAC supports working (ex., working batteries or devices charged), finding them or having access to them, knowing how to use them, and setting them up for specific patients. Additional AAC specific barriers mentioned by one nursing student included patients with vision loss, illiteracy, and low health literacy.

A final barrier communicated by most nursing student participants was patient factors that could hinder communication efforts such as hearing loss, vision loss, cognition, pain, anxiety, low education level, language barrier, history of abuse, or inability to vocalize. It should be noted that these barriers were mentioned as reasons that these populations might be at risk for communication vulnerabilities, thus they are exactly the patients who could benefit from communication supports. 
In addition to barriers, nursing students did provide some facilitators of the use of AAC as well as creative ways that AAC might be used and adapted for a variety of patients in clinical work. A potential facilitator that was highlighted was the personal interaction and depth of knowledge that certified nursing assistants (CNAs) have. Multiple participants mentioned the benefit that might be gained from training CNAs on AAC access and use. A variety of opportunities for AAC teaching and use were identified, though they are not currently used by most hospital facilities or systems. These include using a learning website to provide this type of education to nurses - a main goal of this research, using the TVs in hospital rooms that are already set up to provide education to include an AAC tool that can be accessed using the remote control, and having nurse educators champion using and teaching AAC supports. One student suggested having a larger low-technology AAC board on the white board already in every room and having laser pointers available as an additional access method for patients.

\section{Education}

Educational experience. The nursing students represented three different nursing programs in Tennessee, with three of them in their second semester of their third year, one in their final year with one more semester of clinical experiences left, and another also in their final year of nursing school to complete their Registered Nurse certification (RN; participant already held Licensed Practical Nurse (LPN) and worked under this license previously). All participants reported a variety of coursework and clinical experiences to provide them with a generalized foundation of clinical nursing. One student reported that their program recently began requiring their students to complete 120 hours of academic service learning with a community partner, which this student felt was a great way to learn about a particular type of nursing in more depth while giving back to the community.

Nursing students all had heard of speech language pathology or speech therapy, however, all had learned the discipline's primary role to be swallowing assessments in medical settings or articulation and language therapy in school settings. However, most were familiar with SLPs helping patients communicate post-stroke or when intubated. One nursing student reported AAC being mentioned during one of her nursing courses and reports that she and a few students were able to talk to one of their clinical skill instructors who showed them a pamphlet with basic AAC information. None of the other students reported learning about alternative communication supports in their classes. Therapeutic communication and effective communication were reportedly discussed in mental health courses and throughout simulation experiences.

Educational needs. Nursing student participants reported a willingness to participate in online education modules and felt that they could dedicate time to complete anywhere from two to four 30-minute modules or up to eight hours worth of education. Two of the students reported feeling that a majority of their classmates would be interested, but that there were likely some students who would not be willing to put in additional time to learn something that was not required for their degree. 
Two students reported not feeling prepared to work with and support communication with at-risk or special populations such as DS, ASD, and mental health disorders in kids. Nursing student participants were split with some requesting more information on low-tech AAC, or mid-tech AAC, or high-tech AAC for a variety of reasons, as mentioned above in Types of Communication Supports and Strategies. Additional educational needs voiced included the opportunity for hands on experience with AAC: "Real life, going in and talking to the people because a lot of the times you can read it in the book and you can see the stuff but until you're actually there and you actually see it, you don't know what's going to happen."

Nursing students provided their preference for their highest priority of AAC support need in their clinical work. Their responses varied from low-technology AAC $(n=2)$, mid-technology AAC ( $n=2)$, and high-technology AAC $(n=1)$, with two students each requesting low-technology and mid-technology AAC information. One of the nursing students reported prioritizing low-tech AAC because it could be low cost and available to any patient and, "that's a lot easier to use and that's a lot more accessible and you could have those stapled together at the nurses station...not everybody's going to have an app on their phone, not everybody's going to understand how to use an app on their phone." Reasoning for mid-technology AAC information highlighted the cost and ability to personalize the device and have the potential for digitized voice output. And the expressed reasoning for a preference for high-technology AAC included the adaptability and "how much you can do with it."

\subsection{Quantitative Results}

In addition to the qualitative data collected, quantitative data was collected and analyzed with results providing objective measures of the impact of the online education modules. Two additional research inquiries were addressed through the analysis and interpretation of the quantitative data as discussed in this section.

RI-2. Based on pre- and post-test results from the computer-based learning modules, how do pediatric nurses and nursing students':
a. Knowledge of AAC change?
b. Self-efficacy using AAC change?

\subsubsection{Knowledge}

All three modules included a pre- and post-test to assess participants' knowledge at the outset and following education. Sixty-nine participants completed all three modules; however, two participants did not respond to pre-test items for module 1 resulting in a sample size of 67 . Their data was not included in the repeated measures analysis of variance (ANOVA) for module 1 but all 69 participants had repeated measures data analyzed for modules 2 and 3 . Pre-test and post-test questions were the same for each module with 
module 1 including 10 possible points, module 2 including 15 possible points, and module 3 with 10 possible points. Modules 2 and 3 included new knowledge questions as well as one to two retention questions to assess carryover of information from module to module as well as from pre-test to post-test. Retention questions are included in the total pre-test and post-test scores for modules 2 and 3. Participants demonstrated greater than $95 \%$ accuracy on pre-test and post-test retention questions for modules 2 and 3 thus they were included in the overall pre- to post-test calculations.

The assumptions for the Generalized Linear Model (GLM) were assessed including normality, sphericity, and homogeneity of variance for each module. Normality was assessed with visual analysis of Q-Q Plots with all pre-test and post-test variables meeting the normality assumption (Field, 2013). Skewness and kurtosis were also assessed and found to be with normal ranges for all but the pre-test for module 2 with values just outside the accepted range. The assumption of sphericity was met as there were only two dependent variables for each of the three modules (Field, 2013). The homogeneity of variance was assessed through Levene's test. Modules 1 and 3 met the assumption of homogeneity of variance with non-significant Levene's test results. Module 2, however, indicated a violation of the homogeneity of variance assumption based upon the mean for the pre-test $p=.036$. This may indicate slight concerns for the normality and homogeneity of variance for the module 2 pre-test measures, however, the repeated measures ANOVA provides a robust analysis of the data which accounts for some variation in the normality and homogeneity of variance.

A repeated measures ANOVA was completed for each of the three modules with the results in Table 4.2. All three modules were assessed for differences from pre- to posttest based upon role as a pediatric nurse or nursing student and overall. Module 1 and 2 indicated no statistically significant difference between pediatric nurses and nursing students for pre- to post-test change. All three modules indicated a statistically significant improvement in score based upon time (pre- to post-test) as explained below.

Table 4.2: Pre- and Post-Test Results.

\begin{tabular}{lcccccc}
\hline Test Scores & $\mathbf{n}$ & $\begin{array}{c}\text { Mean } \\
\text { Square }\end{array}$ & $\boldsymbol{d f}$ & $\boldsymbol{F}$ & $\boldsymbol{p}$ & $\eta^{2}$ \\
\hline Module 1 & 67 & 531.28 & 1 & 323.69 & $<.001$ & .83 \\
Module 2 & 69 & 64.21 & 1 & 23.52 & $<.001$ & .26 \\
Module 3 & 69 & 65.08 & 1 & 84.16 & $<.001$ & .53 \\
\hline
\end{tabular}

GLM assumptions were met for normality, sphericity, and homogeneity of variance for the pre-test and post-test measures for module 1 as discussed above. The one-way withinsubjects ANOVA indicated that test scores for module 1 were significantly different from pre to post, $F(1,65)=392.69, p<.001, \eta^{2}=.83$. The average pre-test score $(M=5.45)$ increased in the post-test $(M=9.55$; Figure 4.1$)$. The $\eta^{2}$ for this module indicates a large effect size in 


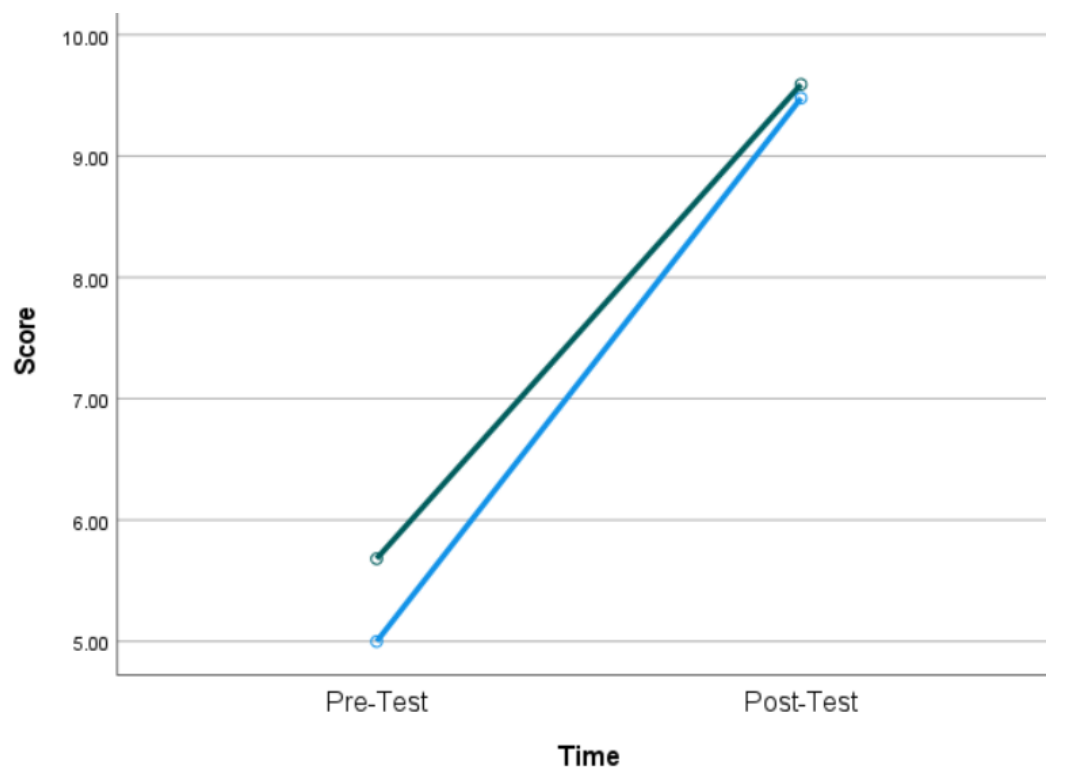

Figure 4.1: Module 1 Pre- to Post-Test Scores.

Nursing Students, _ Pediatric Nurses

addition to a statistically significant change from pre-test to post-test (Cohen, 1988). Time was a significant factor from pre-test to post-test, however, there was not a statistically significant difference in scores or change in scores based upon group membership for this module. Pediatric nurses and nursing students began with approximately the same pre-test scores and improved at approximately the same rate of knowledge increase to reach around the same post-test score.

The one-way within-subjects ANOVA indicated that test scores for Module 2 from pre to post were significantly different, $F(1,67)=23.52, p<.001, \eta^{2}=.26$. The average pre-test score $(M=12.52)$ increased in the post-test $(M=13.91$; Figure 4.2$)$. The $\eta^{2}$ for this module indicates a large effect size in addition to a statistically significant change from pre-test to post-test (Cohen, 1988). A limitation can be found in the previously mentioned significant result in Levene's test $(p=.036)$.

Again for module 2, time was a significant factor from pre-test to post-test, with both groups increasing their knowledge at a similar rate. Pediatric nurse and nursing student scores did not differ significantly.

GLM assumptions were met for normality, sphericity, and homogeneity of variance for the pre-test and post-test measures for module 3 . The one-way within-subjects ANOVA indicated that test scores for module 3 from pre to post were significantly different, $F(1,67)$ $=84.16, p<.001, \eta^{2}=.53$. The average pre-test score $(M=8.26)$ increased in the post-test $(M$ 


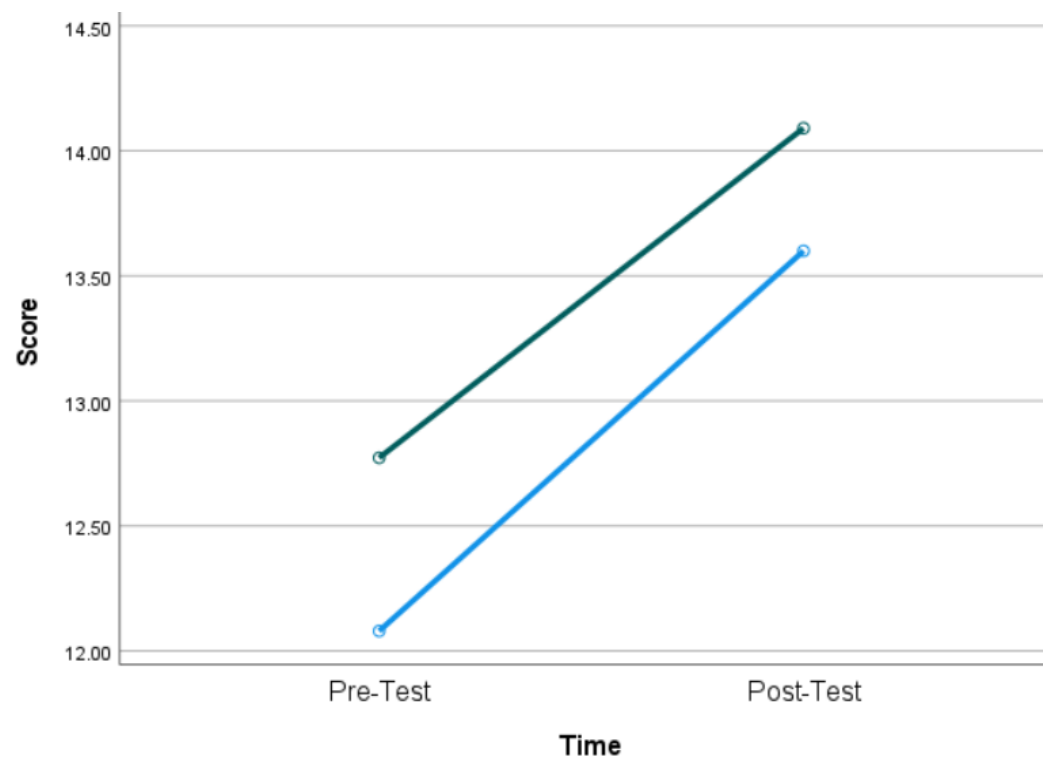

Figure 4.2: Module 2 Pre- to Post-Test Scores.

Nursing Students, _ Pediatric Nurses

\section{= 9.57; Figure 4.3).}

An interaction effect was noted, thus independent samples t-tests were calculated for pre-test and post-test by group. The two groups were found to differ significantly for the pre-test score. The 25 pediatric nurses $(M=7.76, S D=1.54)$ compared to the 44 nursing students $(M=8.55, S D=1.21)$ demonstrated significantly lower pre-test scores, $t(67)=-2.349$, $p=.02$. There was not a statistically significant difference between groups at the post-test $(t(67)=.632, p=.529)$. These results indicate that the pediatric nurses, on average, scored lower on the pre-test compared to the nursing students, but following the education, both groups scored approximately the same on the post-test. The pediatric nurses demonstrated a greater overall increase in score from baseline.

There were also one to two retention questions posed in module 2 and module 3 to determine whether participants were able to carry over the knowledge from the previous module(s). The retention question averages for module 2 were out of five possible points: pre-test $M=4.79, \mathrm{n}=68$ and post-test $M=4.84, \mathrm{n}=69$. Module 3 retention questions were worth three points: pre-test $M=2.88, \mathrm{n}=69$ and post-test $M=2.94, \mathrm{n}=69$. These averages indicated that participants retained much of the information from previous modules for later recall with a slight increase noted from pre-test to post-test for modules 2 and 3.

Open-ended questions were posed throughout the pre- and post-tests with questions such as "List or describe two pediatric populations/diagnoses/situations that could benefit from communication strategies." Examples of pre- and post-test responses to this question 


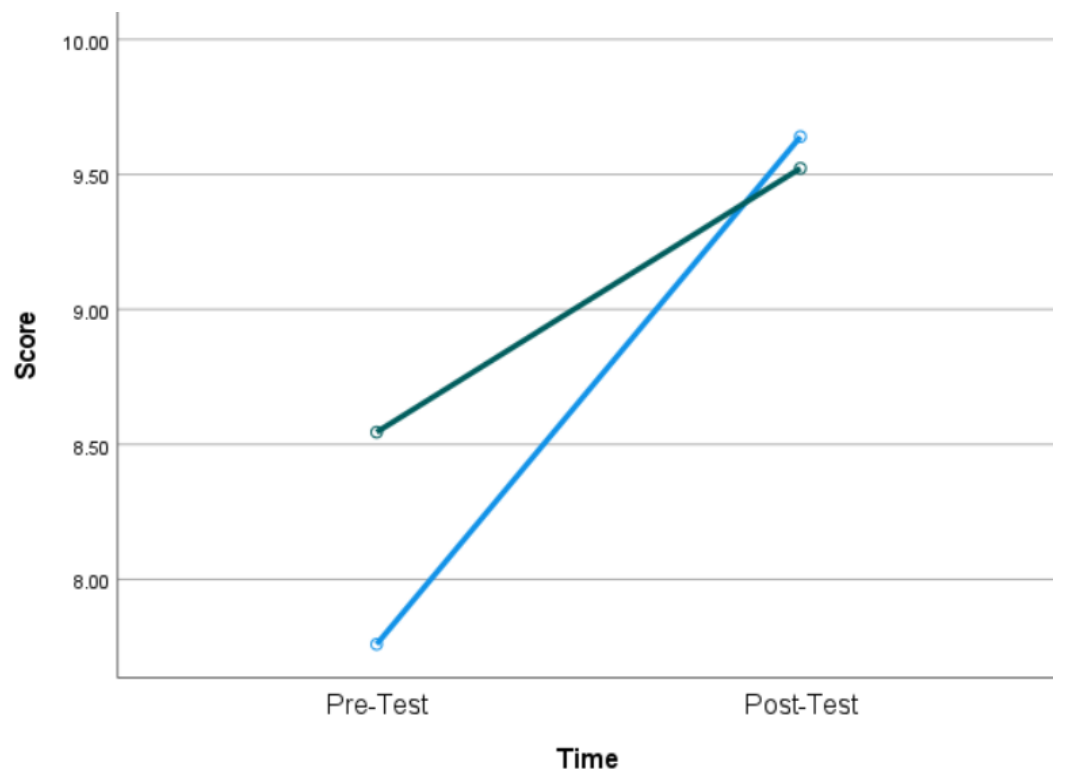

Figure 4.3: Module 3 Pre- to Post-Test Scores.

Nursing Students, _ Pediatric Nurses

from across all three modules included DS, non-native English speaking patients, ASD, ADHD, speech delay, respiratory distress, children who are deaf, children with developmental disabilities, cancer, poverty, cognitive deficits, children who are shy/timid, tracheostomy patients, surgery patients, mental illness, ventilated patients, and intubated patients. In addition to common diagnoses that might benefit from communication strategies, some participants described situations such as end of life decision making, giving a new but hard/scary diagnosis, teaching new information on caring for new medical equipment, or hand-off between healthcare providers between shifts. Participants provided a large variety of patients and situations that they felt might benefit from communication strategies, with most responding they were unsure during the pre-test and then providing the large list of examples above in the post-test.

\subsubsection{Self-Efficacy}

Self-efficacy was measured using a 100-cm sliding scale in the pre- and post-test as well as through the use of a set of validated Likert scales (Kang et al., 2019) in the post-test alone. A repeated measures ANOVA was used to assess the change in perceived self-efficacy for participants from the pre- to post-measure as measured on the sliding scale.

As for the pre-test to post-test measures, the GLM assumptions (normality, sphericity, and homogeneity of variance) were assessed for the self-efficacy measures in the same way as the knowledge variables. Normality was observed through Q-Q plots and skewness 
and kurtosis measures, sphericity verified as there are only two dependent variables, and homogeneity of variance verified through Levene's tests. A synopsis of the self-efficacy data for all three modules can be found in Table 4.3.

Table 4.3: Self-Efficacy Repeated Measures Results.

\begin{tabular}{lcccccc}
\hline Self-Efficacy Scores & $\mathbf{n}$ & $\begin{array}{c}\text { Mean } \\
\text { Square }\end{array}$ & $\boldsymbol{d f}$ & $\boldsymbol{F}$ & $\boldsymbol{p}$ & $\eta^{2}$ \\
\hline Module 1 & 64 & 44774.53 & 1 & 97.27 & $<.001$ & .61 \\
Module 2 & 56 & 28337.75 & 1 & 77.20 & $<.001$ & .54 \\
Module 3 & 60 & 81896.43 & 1 & 197.65 & $<.001$ & .76 \\
\hline
\end{tabular}

As for the pre- and post-test data, all three modules were assessed for differences from pre to post based upon role as a pediatric nurse or nursing student and overall. All three modules found a statistically significant difference between pediatric nurses and nursing students for pre to post for self-efficacy, and modules 2 and 3 each found an interaction effect with statistically significant differences based upon group and time. All three modules indicated a statistically significant improvement in score based upon time (pre to post) as explained below.

Module 1 perceived self-efficacy measures indicated a statistically significant increase from pre to post, $F(1,62)=97.27, p<.001, \eta^{2}=.61$. The average pre-test score for perceived self-efficacy $(M=45.23)$ increased in the post-test $(M=85.07)$. There was a statistically significant difference based upon group $\left(F(1,62)=5.82, p=.02, \eta^{2}=.09\right)$, with both groups' perceived self-efficacy increasing at a similar rate from pre to post. Pediatric nurse pre-measure average was 52.00 which increased to 89.67 compared to nursing student pre-meausre average of 38.47 increasing to 80.47 . The average increase of 40 points from pre to post in addition to the statistically significant result and a large effect size would indicate an increase in perceived ability to understand and use the information on yes/no strategies, wait time, and recasting presented in module 1 (Figure 4.4; Cohen, 1988).

Module 2 perceived self-efficacy measures indicated a statistically significant increase from pre to post, $F(1,54)=77.20, p<.001, \eta^{2}=.54$. The average pre-test score for perceived self-efficacy $(M=49.22)$ increased in the post-test $(M=82.48)$. The 33 point average increase from pre to post-test, statistically significant result, and large effect size indicate an increase in perceived ability to understand and use the information presented on choice making and simple sign language in module 2 (Figure 4.5; Cohen, 1988).

There was an interaction effect and a statistically significant difference for self-efficacy rating based upon group that was further explored with the use of independent samples t-tests. The 21 pediatric nurses $(M=63.10, S D=27.19)$ compared to the 37 nursing students $(M=33.30, S D=28.69)$ demonstrated significantly higher pre-test self-efficacy scores, $t(56)=$ $3.87, p<.001$. There was a non-significant difference between groups at the post-test $(t(64)=$ $1.05, p=.30)$. These results indicated that the pediatric nurses, on average, scored higher on 


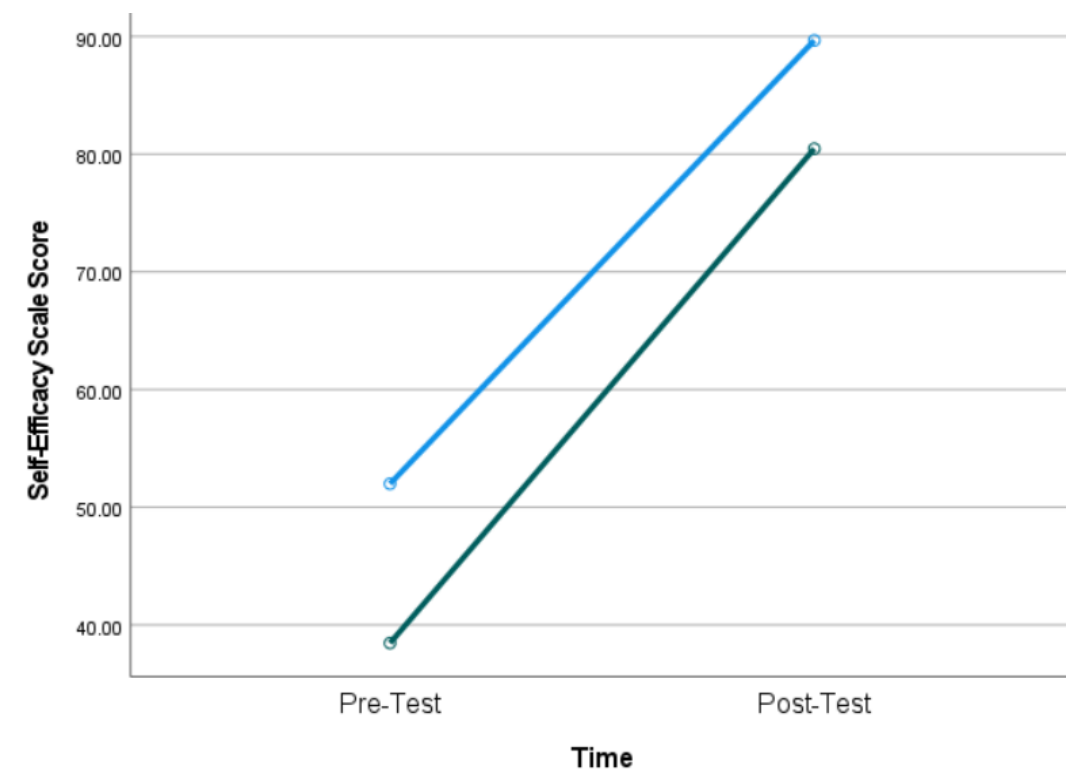

Figure 4.4: Module 1 Self-Efficacy Scale Scores.

Nursing Students, _ Pediatric Nurses

the initial self-efficacy scale compared to the nursing students, but following the education, both groups scored approximately the same on the self-efficacy measure. The nursing students demonstrated a greater overall increase in perceived self-efficacy from baseline.

Module 3 perceived self-efficacy measures indicated a statistically significant increase from pre to post, $F(1,58)=197.65, p<.001, \eta^{2}=.76$. The average pre-test score for perceived self-efficacy $(M=30.40)$ increased in the post-test $(M=85.17)$. The average increase of 55 points from pre to post-test, the statistically significant result, and the large effect size would indicate a noted increase in perceived ability to understand and use the information on partner assisted scanning presented in module 3 (Figure 4.6; Cohen, 1988).

There was another interaction effect for module 3 with group membership significantly impacting self-efficacy scores as well as time. Independent samples t-tests were conducted to further examine the relationships between the variables. Similar to module 2 , the 23 pediatric nurses $(M=37, S D=35.99)$ compared to the 39 nursing students $(M=$ $20.28, S D=27.15$ ) demonstrated significantly higher perceived self-efficacy scores at the initial assessment, $t(60)=2.07, p=.04$. There was not a statistically significant difference in perceived self-efficacy following the education, $t(64)=1.51, p=.25$. Similar to module 2 , these results indicate that pediatric nurses had a higher perceived self-efficacy from the outset than nursing students but that following participation in the CBLs, both groups rated their perceived self-efficacy to be approximately the same. 


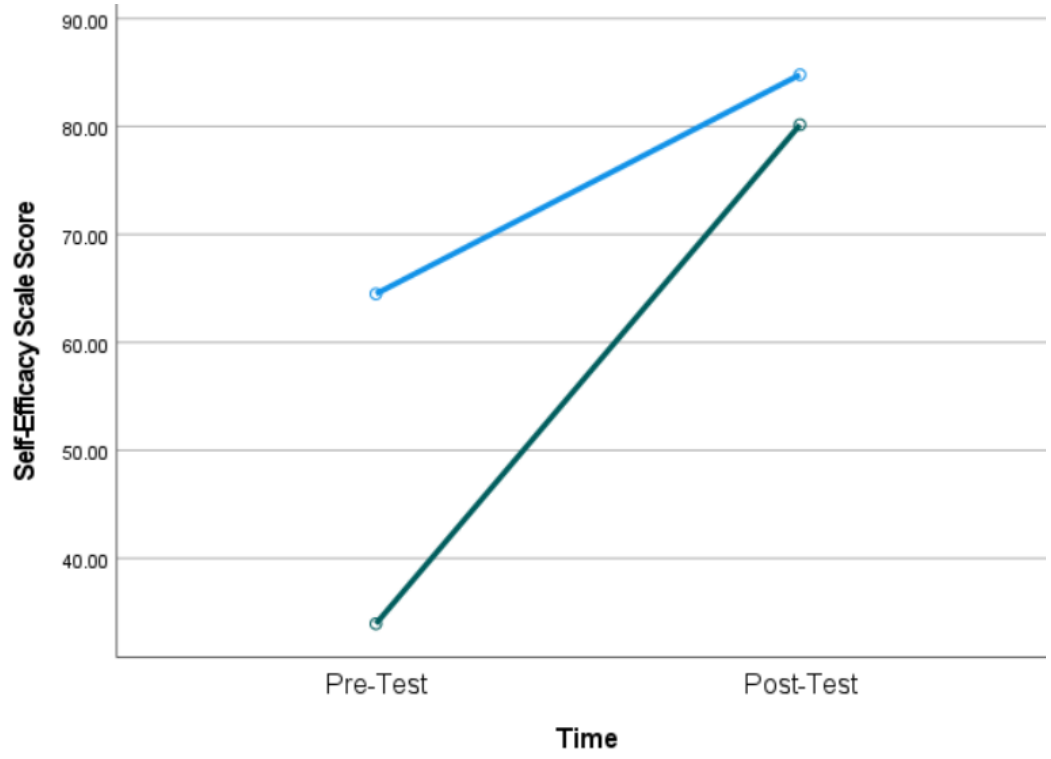

Figure 4.5: Module 2 Self-Efficacy Scale Scores.

Nursing Students, _ Pediatric Nurses

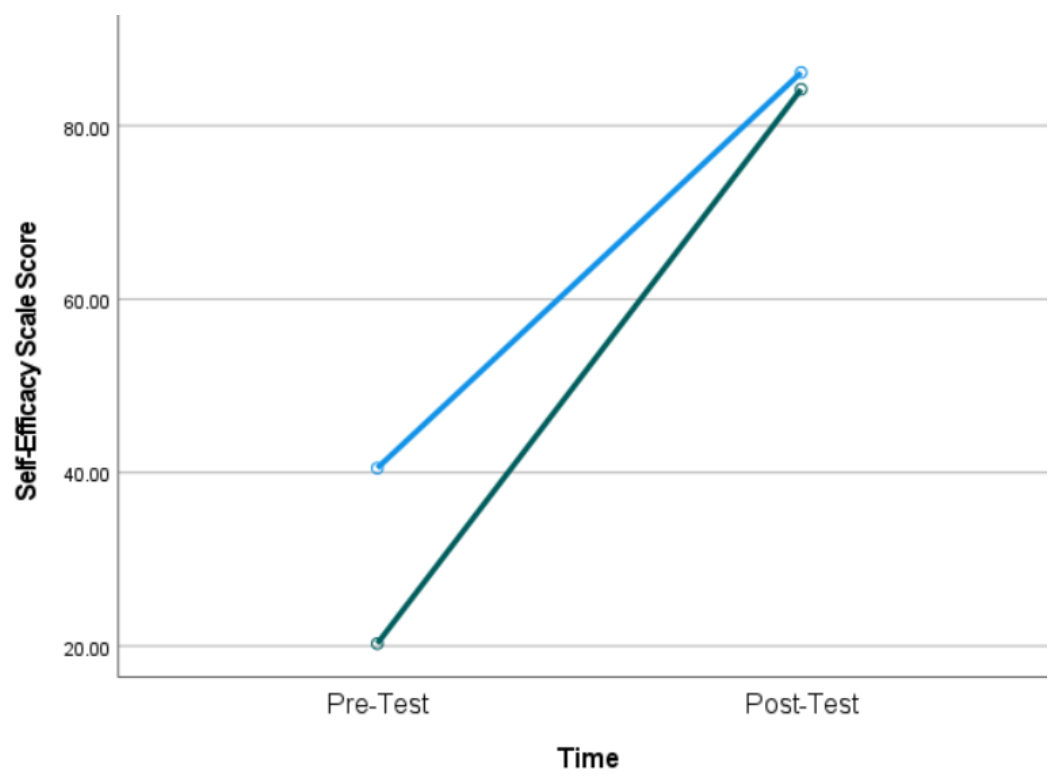

Figure 4.6: Module 3 Self-Efficacy Scale Scores.

— Nursing Students, _ Pediatric Nurses 
RI-3. Based on post-assessment results from the CBLs, how do pediatric nurses and nursing students rate the:
a. Adapted Learning Self-Efficacy Scale (Cognitive, Affective, Psychomotor)
b. Relevance of Content
c. Effectiveness of the Educator and Method

Reliability was assessed prior to statistical analysis. Two items in the relevance scale and four in the effectiveness scale were negatively worded and reverse coded prior to calculating reliability. Acceptable reliability $(\alpha>.70)$ was found for all scales except for the affective scale of the L-SES for module $1(\alpha=.623)$ and $2(\alpha=.568)$ and the psychomotor scale for module $1(\alpha=.639)$. For the affective scale, it was indicated through item analysis that the removal of the first item, "compare this education module to other continuing education you have completed - I think I spent more time on this module than on others," would positively impact the overall scale reliability. For consistency, this initial item was removed from all three module scales. An item analysis for the psychomotor scale for module 1 indicated some reliability would be gained through removing item one in that scale which states, " I can precisely imitate the instructor's steps and actions of wait time." A complete list of reliability measures can be found in Table 4.4.

Table 4.4: Reliability Scores for Likert Scales.

\begin{tabular}{lccccc}
\hline Reliability Scores & Cognitive & Affective & Psychomotor & Relevance & Effectiveness \\
\hline Module 1 & $\alpha=.91$ & $\alpha=.76^{*}$ & $\alpha=.68^{*}$ & $\alpha=.80$ & $\alpha=.83$ \\
Module 2 & $\alpha=.88$ & $\alpha=.73^{*}$ & $\alpha=.91$ & $\alpha=.82$ & $\alpha=.85$ \\
Module 3 & $\alpha=.90$ & $\alpha=.88^{*}$ & $\alpha=.88$ & $\alpha=.85$ & $\alpha=.87$ \\
\hline
\end{tabular}

* Adapted scale

A multivariate analysis of variance (MANOVA) was run for each of the three modules to determine whether differences existed in the five Likert scales between the two groups, pediatric nurses and nursing students, while reducing Type I error (a false positive or incorrectly rejecting the null hypothesis). The assumptions were assessed for each module prior to analysis of the MANOVA results and found to be satisfactory.

The MANOVA allowed the comparison of the independent variable of group (pediatric nurse vs. nursing student) across all five scales (cognitive, affective, psychomotor, relevance, and effectiveness). Module 1 yielded a non-significant MANOVA with a $p$-value $>.05$.

The MANOVA for Module 2 indicated a significant result, (Pillai's V $=.27, F(5,63)=$ $\left.4.67, p=.001), \eta^{2}=.27\right)$. Further examination of the univariate one-way ANOVAs indicated a significant difference based upon group for two scales: relevance $(F(1,68)=19.27, p<.001$, $\left.\eta^{2}=.22\right)$ and effectiveness $\left(F(1,68)=3.97, p=.05, \eta^{2}=.06\right)$. The relevance scale indicated 25 
pediatric nurses $(M=4.02, S D=.69, S E=.10)$ rated the relevance mildly lower compared to the 44 nursing students $(M=4.56, S D=.32, S E=.07)$. The nursing students may have found the information slightly more relevant to their clinical rotations, clinical work, or education than pediatric nurses who participated. The effectiveness scale for the same module found that 25 pediatric nurses again rated the effectiveness slightly lower $(M=4.48$, $S D=.68, S E=.10)$ than the 44 nursing students $(M=4.73, S D=.36, S E=.08)$. The pediatric nurses indicated that the modules were overall very effective though slightly less so than the nursing students. This may be due to the fact that nursing students are more familiar with online learning than pediatric nurses.

A MANOVA was run for the same five scales for Module 3 which provided a significant result (Pillai's V $=.24, F(5,63)=3.96, p=.004), \eta^{2}=.24$ ). Further examination of the one-way ANOVAs for the five scales indicated a significant difference for the relevance $\left(F(1,68)=13.04, p<.001, \eta^{2}=.16\right)$ and psychomotor scales $\left(F(1,68)=16.17, p<.001, \eta^{2}\right.$ $=.19)$. The relevance scale reported 25 pediatric nurses rate relevance slightly lower $(M$ $=3.91, S D=.10, S E=.12)$ compared to the 44 nursing students $(M=4.44, S D=.49, S E$ $=.09$ ). This indicated that the nursing students may have found the information mildly more relevant than pediatric nurse participants, thought both reported they somewhat agreed with relevance items. The psychomotor scale provided information regarding the 25 pediatric nurse participants' $(M=4.25, S D=.55, S E=.09)$ perception of the effectiveness of the education compared to the 44 nursing students $(M=4.69, S D=.35, S E=.07)$. Again, both groups indicated an overall positive (somewhat agree to strongly agree) rating, with the nursing students responding with a higher overall positive rating regarding this education.

\subsubsection{Adapted L-SES}

Each of the three sub-scales of the Learning Self-Efficacy Scale (L-SES) were initially assessed for reliability using Cronbach's alpha for each module. All three scales demonstrated good reliability as determined by Cronbach's alpha scores ranging from .684 to .910. As mentioned above, the affective scale for modules 1 and 2 were assessed without the inclusion of the first item, as was the psychomotor scale for module 1. It was decided that the affective scale for module 3, despite having an acceptable Cronbach's alpha with all four items, would only include the same three items for analysis to maintain consistency with modules 1 and 2.

The average scale scores for each module can be found in Table 4.5. L-SES Scale averages across participants for each module indicated Likert scale values of somewhat agree to strongly agree for the cognitive and psychomotor scale. The averages for the affective scale of the L-SES ranged from 3.33 to 3.70 indicating an average of neutral to somewhat agree. This scale score falls slightly below the hypothesized rating of somewhat to strongly agree with an average score below 4 . This scale was reportedly confusing to a pilot partQI = qualitative interview, $\mathrm{QFGs}=$ qualitative focus groups, $\mathrm{GC}=$ group comparison, $\mathrm{D}=$ descriptive, $\mathrm{BP}=$ best practices, $\mathrm{RA}=$ retrospective analysis, $\mathrm{PACU}=$ 
post-anesthesia care unit, ICU = intensive care unit, Day-Sx = day surgeryicipant, but ultimately it was included in the implementation as it was a part of the validated L-SES.

Table 4.5: Post-Test Scale Averages.

\begin{tabular}{llccccc}
\hline Module & \multicolumn{1}{c}{ Scale } & $\boldsymbol{N}$ & Minimum & Maximum & Mean & SD \\
\hline $\mathbf{1}$ & L-SES: Cognitive & 69 & 1.00 & 5.00 & 4.52 & .66 \\
& L-SES: Affective & 69 & 1.67 & 5.00 & 3.64 & .67 \\
& L-SES: Psychomotor & 69 & 4.00 & 5.00 & 4.69 & .36 \\
& Relevance & 69 & 2.29 & 5.00 & 4.40 & .51 \\
$\mathbf{2}$ & Effectiveness & 69 & 3.00 & 5.00 & 4.54 & .51 \\
& L-SES: Cognitive & 69 & 3.00 & 5.00 & 4.65 & .52 \\
& L-SES: Affective & 69 & 1.67 & 5.00 & 3.70 & .72 \\
& L-SES: Psychomotor & 69 & 2.00 & 5.00 & 4.57 & .55 \\
& Relevance & 69 & 2.00 & 5.00 & 4.36 & .55 \\
& Effectiveness & 69 & 2.75 & 5.00 & 4.64 & .51 \\
& L-SES: Cognitive & 69 & 3.00 & 5.00 & 4.68 & .46 \\
& L-SES: Affective & 69 & 1.67 & 5.00 & 3.45 & .92 \\
& L-SES: Psychomotor & 69 & 3.00 & 5.00 & 4.53 & .48 \\
& Relevance & 69 & 2.43 & 5.00 & 4.25 & .64 \\
& Effectiveness & 69 & 1.75 & 5.00 & 4.53 & .61 \\
\hline
\end{tabular}

In the open-ended questions found in the post-test for each module, a participant responded, "There was a question that asked about this module in comparison to the other modules. I wasn't able to complete that question accurately because I have yet to do the other modules." This indicates some confusion with the affective scale which included items like, "I think I gained more from this module than others." The initial intent of the scale was to compare this education to other education, not to compare the three modules to each other. This may be a reason that the affective scale results had lower reliability than the other scales.

The cognitive scale averages ranged from 4.52 to 4.68 indicating participants strongly agreed with their perceived grasp of the information presented in the education modules. The psychomotor scale averages ranged from 4.53 to 4.69 indicating, similarly to the cognitive scale, an overall strongly perceived ability to utilize and act out the communication strategies taught in each of the CBLs. Both of these results for the cognitive and psychomotor scales met the hypothesized result of an overall rating of somewhat to strongly agree (score of greater than or equal to 4).

\subsubsection{Relevance of Content}

The relevance of the content for each module was measured and found to range from 4.25 to 4.40 indicating that participants somewhat to strongly agreed with the content relevance 
for their current or future clinical work. This result is in line with the hypothesized result of an overall relevance rating of somewhat to strongly agree (score of greater than or equal to $4)$.

\subsubsection{Effectiveness of the Educator and Method}

The effectiveness scale averages indicated strong agreement from participants that the method of education and the educator were effective at communicating information with scale averages ranging from 4.53 to 4.64 . This result is in line with the hypothesized result of an overall effectiveness rating of somewhat to strongly agree (score of greater than or equal to 4$)$.

\subsubsection{Clinical Application}

In addition to the multiple choice, multiple option, true false, and Likert scale questions in the pre-test and post-test, all three modules had open-ended post-test questions probing potential clinical use or benefits following the CBL participation.

\section{Module 1: Wait time and recasting}

Module 1 content included an introduction into communication, AAC, and communication supports and strategies, specifically wait time and recasting. Participants were asked to provide responses to how they might use this new information in their clinical work. Following this CBL, participants were asked one change that they would make in their clinical practice as a result of what they learned in the module.

Participant responses varied but included the use/provision of wait time and observe, wait and listen $(\mathrm{OWL} ; \mathrm{n}=56)$. A common sentiment participants communicated was feeling like they do not provide adequate time for patients to respond as evidenced by comments such as, "I think that I haven't been giving patients enough time to consider the question and respond." Another stated, "'I will make sure to allow the patients additional time to respond to the questions I ask instead of thinking they aren't listening or paying attention to me." Additional responses regarding the importance of wait time with kids were evidenced by, "I will use this more because I now realize how people who are sick and/or children in general need more time to process what was just presented to them." Another comment indicating the role that time plays in medical care was, "I find myself not allowing any time after asking my patient a question in order to be speedy and complete tasks in a timely manner. This changes my perspective on how much time I need to be spending with patients." The role this plays specifically with pediatric patients was highlighted: "I think waiting for a response is very appropriate with the pediatric population. I like this idea a lot". Some participants mentioned a plan to incorporate rephrasing and recasting into their clinical work as an additional communication support to patients $(n=10)$.

In addition, a number of participants responded that they felt they would be better listeners $(\mathrm{n}=8)$, be more aware of no-tech AAC $(\mathrm{n}=2)$, and work at providing visual cues 
when giving patients options $(n=1)$. In addition, participants were asked how they felt their patients would benefit from this education. Responses overwhelmingly focused on improvements in communication and care $(n=50)$. A large number of nurses also felt that this information would help them to spend more focused time with their patients as well as to assist them in taking the time to understand and respond to their patients' needs $(n=24)$.

Participants reported they felt their patients would benefit because they would be better equipped to do their job $(n=9)$, and that patients would feel more understood and cared for, with an increase in trust $(n=8)$. One participant said, "I think my patients' sense of dignity will be upheld with these techniques. By providing longer wait times, I am showing them that I care and understand they are still capable of providing a response." Another participant said, "I hope that they [patients] will feel empowered to speak and be involved in care since I am making the conscious effort to wait for a response and not rush the conversation." A participant stated she felt a direct benefit to her patients would be, "They will feel that they have plenty of space and time to express themselves." A final benefit was the potential for decreased anxiety, with patients feeling more in control $(n=2)$.

When asked the barriers that could prevent participants from implementing what they had learned, a number reported they were unsure or there were none $(n=15)$. This may be due to student participation which largely includes clinical rotations which change frequently, thus it may have been difficult to identify consistent barriers. One barrier mentioned was no access to AAC $(n=17)$. In a similar vein was no access to specialists such as SLPs or translators $(n=3)$ and language barriers $(n=6)$.

A common barrier mentioned in the literature as well as in the interviews and focus groups for this research resurfaced in these open-ended questions: time $(n=10)$. Examples of time-related barriers included busy shifts, limited staff, and emergency situations. Another commonly mentioned barrier was lack of education or knowledge $(n=7)$ including a comment regarding a limited focus on AAC in nursing education. Additional concern was raised regarding the amount of time for some students to have an opportunity to use these communication strategies based on their clinical rotation or site $(n=2)$ and what to do if a strategy does not work $(\mathrm{n}=1)$.

Additional barriers included families who may be uncooperative or patients who may be unmotivated or aggressive $(n=4)$. A final barrier was the concern over reading facial expressions with the current widespread use of masks $(n=1)$ which hinders the ability to effectively expressively and receptively exchange information through facial expressions.

In addition to barriers, a variety of facilitators were mentioned with the most common being the need for supervisor/management/co-worker buy-in and support for AAC to be truly effective $(n=31)$ and a work culture supportive of the time needed to implement AAC $(n=1)$. Other individuals that participants felt would be communication support facilitators included parents $(\mathrm{n}=3)$, and feedback from patients, clinical instructors, and other nurses $(n=4)$. One nursing student reported a high value placed on communication in her nursing program which she felt was a facilitator to using the information provided. 
Additional facilitators to the no-tech AAC taught, were the ability to practice using the strategies as well as some background knowledge and previous experience that some brought to the table $(n=7)$. Accessibility to AAC on each unit $(n=3)$, SLP visits, evaluation, recommendations, and general presence of SLPs $(n=4)$, and posted notes and reminders to refer to speech language pathology $(n=2)$ were mentioned. Though these were mentioned as facilitators, it appeared that some, such as the desire for notes and reminders to refer and accessibility to SLPs were things that participants felt would be facilitators, but that were not necessarily in place at that time. Additional desired facilitators were ongoing training, education, and practice for all nurses $(n=7)$, and a translation method for non-English speaking patients and families $(n=1)$.

Participants were provided with an open-ended section at the end of each module where they could place additional information and questions. A number of participants left this question blank. Of those that responded, some reported no questions ( $n=19)$, while a couple requested additional information about AAC, SLP role, and sign language $(n=2)$. A final small group of individuals provided positive comments regarding the content and effectiveness of the educator and education $(n=4)$.

\section{Module 2: Choice making and simple sign language}

Module 2 focused on different ways to provide options for choice making including objects, pictures, or communication boards. Simple sign language was also introduced as a notechnology option for communication between healthcare provider and patient.

When participants were asked for one change they would make in their clinical practice as a result of what they learned in the module, many responded with the use of sign language and/or the use of communication boards $(n=37)$ as they had seen demonstrated in this education video. Additionally, many participants mentioned they planned to use hand gestures and choice making $(n=6)$ and use objects for choices $(n=6)$. A number of participants reported they planned to provide less choices to patients $(n=4)$ as they felt they had likely given too many in the past. Additional comments included the desire to continue using wait time strategies as mentioned in the last module, and low-technology AAC strategies such as a whiteboard an marker were provided $(n=1)$.

Participants felt that their patients may benefit from the information they received by getting better care through more effective communication exchanges $(n=35)$. The nurses and students also wrote often of their hope that from this they will be able to support patients' communication, allowing them to feel more in control of their care, comfortable, and empowered $(n=10)$. They also said they hoped that patients would feel seen and heard $(n=3)$, with a result of increased trust and less fear $(n=2)$. One participant also stated they felt there might be less patient stress and anxiety in choice making $(n=1)$ from what they know now.

In addition, participants felt their patients may be able to use simple sign more

effectively $(n=2)$. One nurse pointed out the benefit that these strategies may have for 
adult patients as well as pediatrics. Two participants felt that the no-technology aspect of these strategies may make it easier for some patients to interact with these strategies.

Participants were asked to identify some barriers to using the strategies for choice making and simple sign language. A number of participants could not think of any barriers $(n=12)$, with one person stating, "None for this low technology since I will be provided with communication boards at the end of this training."

Other participants did identify some barriers to using the taught strategies with the most often mentioned being access to resources such as communication boards or SLPs ( $\mathrm{n}=$ 35). Lack of in-depth training on AAC or sign language $(n=4)$, limited experience with AAC $(n=1)$, and lack of unit support $(n=2)$ were mentioned as barriers. Time was also mentioned $(n=2)$ in addition to cultural and linguistic barriers $(n=3)$, lack of widespread knowledge about AAC ( $n=1)$, and difficulty recalling multiple signs $(n=1)$.

Patient characteristics were also mentioned as potential barriers such as a lack of patient buy-in (i.e., patients may not have the patience to learn sign language, adult patients might think communication boards are silly; $n=2)$, patient medical status $(n=3)$, or patient abilities such as reduced vision or cognition $(n=2)$. In addition to barriers, a number of facilitators were also provided. As seen in module 1, the support of management and co-workers was a common facilitator $(n=24)$, as well as access to speech therapy $(n=4)$. Additionally, access to materials such as communication boards and dry erase markers $(\mathrm{n}=$ $18)$ and additional training and education $(n=11)$ were reported facilitators. Both of these facilitators may fall into the category of desired facilitators rather than facilitators currently in place. One participant stated, "I think it would be beneficial if all hospitals/clinics etc. has some sort of training in this communication," and another said, "if nurse educators taught incoming nurses these techniques, we could implement these from the beginning."

Other facilitators related to choice making and simple sign language included the benefit of visual aids to patients $(n=1)$ and that these strategies are low cost and easy to use $(\mathrm{n}=1)$. A few potentially desired facilitators included working with Child Life to get access to communication boards $(n=1)$, having a list of simple signs handy on the unit $(n=1)$, being able to watch other nurses use AAC $(n=1)$, having time during a shift to implement AAC $(n=1)$, and having parents of patients available to support communication $(n=1)$. Two participants reported they could not identify any facilitators at this time.

The final open-ended question provided participants the opportunity to ask additional questions or request information they would like. Many participants reported that they did not have additional questions $(n=20)$. A few requested flyers to share with other nurses $(n=2)$, or more information or resources for communication boards $(n=1)$. One nurse requested written information to review in conjunction with the videos $(n=1)$, and another requested more information on sign language and choice making $(n=1)$. The requests for more information and resources were met as synopses of all module content and references were provided to all participants following the completion of the modules. Additional positive comments regarding the benefit of this education were voiced as two 
participants felt the basic sign language information was useful with one stating, "that was great, thank you! I myself have a congenital hearing deficiency and cannot yet afford hearing aids (hopefully the end of this year), so sometimes I mishear things at work. Simple signs will be beneficial for children that are like me!"

\section{Module 3: Partner-assisted scanning}

Module 3 discussed partner-assisted scanning (PAS) including appropriate populations and situations that may benefit from PAS and how to implement it. Following the completion of this CBL, participants were asked to report one change that they would make in their clinical practice as a result of what they learned. Many participants stated they would use more methods of communication with patients $(n=16)$, such as communication boards $(n=16)$, and feel they are better equipped to communicate effectively $(n=10)$. A few participants also mentioned greater awareness of how to communicate with patients $(n=3)$, with two participants mentioning they plan to create a unit specific low-technology AAC board to use in their clinical practice. One participant, following this module, did create a low-technology communication board using the provided template (Figure 4.7).

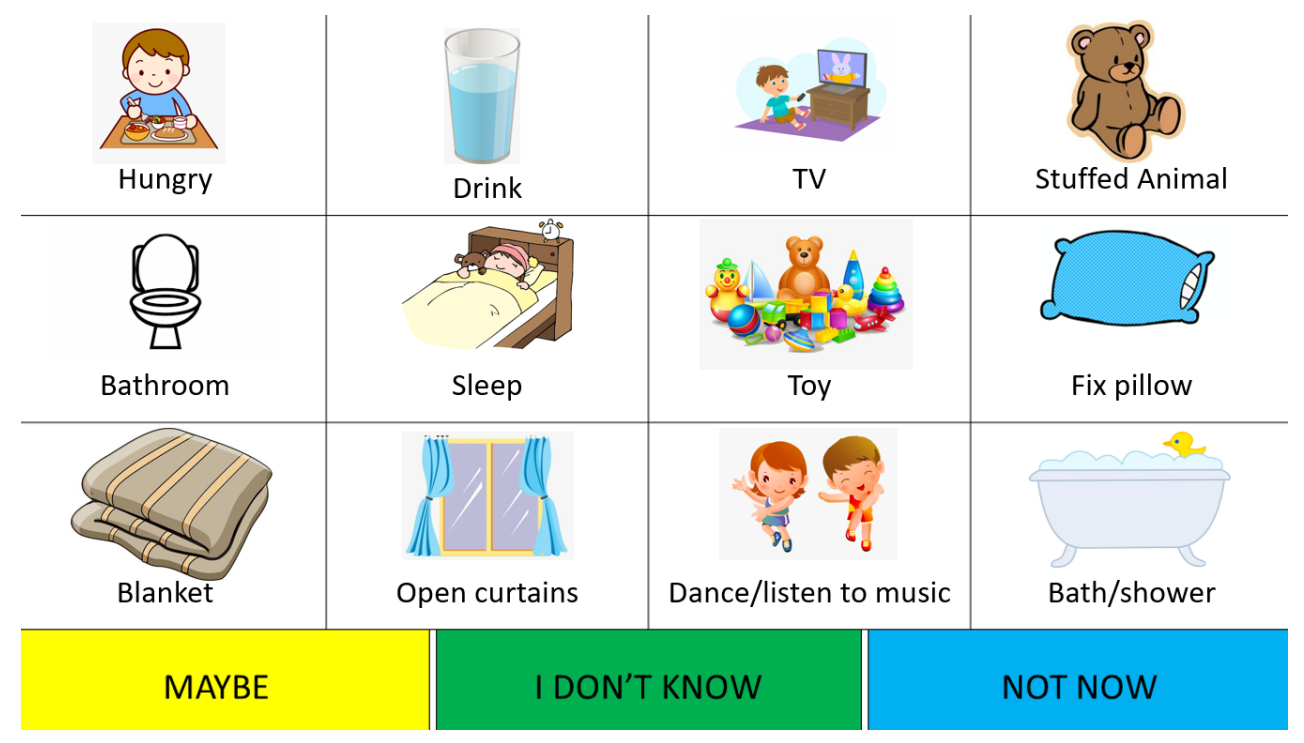

Figure 4.7: Low-Technology Board Created by Participant.

Some participants reported they plan to take time to adapt communication supports to fit patients' needs $(n=3)$, such as giving patients more time $(n=2)$, being more patient $(n=2)$, feeling better prepared to deal with time constraints on the unit $(n=1)$, and provide more careful assessments to determine the best method of communication $(n=2)$. One nurse reported a desire to provide education and training to co-workers in the NICU, and another reported planning to communicate with other healthcare providers about what 
communication techniques work best with patients. Two participants reported no change to their clinical practice, and another stated they provide immunizations for healthy children and are unsure whether these supports would be useful.

Participants reported a variety of perceived patient benefits with the primary being, as seen in modules 1 and 2, better or easier communication and thus better care and patient experience $(n=38)$. Following this module, a number of participants reported the emotional impact that this training may have on patients with six nurses reporting they felt patients will feel heard and that someone is willing to listen, and six more reporting patients may be more confident in decision-making and communication attempts. Patient autonomy $(n=5)$, patient needs being met $(n=5)$, increased patient engagement in their care $(n=1)$, patients being happier and more comfortable $(n=1)$, and patients receiving more holistic care $(n=$ 1) were all highlighted. Additionally, one participant felt patients would be encouraged by better communication $(n=1)$ with a greater understanding of their options, more easily managed pain, and ability to be calm $(n=1)$. An additional benefit was more resources and additional ways to effectively communicate with patients $(n=2)$ and more adaptability of nurses to communicate with patients $(n=1)$. One participant was unsure of patient benefits following this CBL and two others reported no predicted benefits to patients.

Though a number of participants did not identify any barriers to the use of PAS ( $\mathrm{n}=$ 8 ), others were able to provide examples of barriers to using this information, including access to AAC and materials or lack of knowledge on where to find those materials $(n=24)$. An additional seven participants reported some but limited access to AAC and materials.

Some participants reported that the use of PAS would not apply to their patient population or work setting $(n=2)$, that they had no experience as they were still in school $(\mathrm{n}=1)$, or had a lack of program or unit support $(\mathrm{n}=4)$. Time was a highlighted barrier ( $\mathrm{n}$ $=4)$, as well as knowledge and training barriers $(n=3)$. Patient medical or cognitive status $(\mathrm{n}=4)$ as well as language, literacy, and visual barriers $(\mathrm{n}=3)$ were mentioned. Patients unable to consistently provide a yes/no response would be a barrier to the use of PAS ( $\mathrm{n}=$ $4)$ as well as if someone were unable to recall how to implement PAS ( $n=1)$ or in a setting where PAS and the use of AAC was not taught or used $(n=1)$.

In contrast to the above mentioned barriers were a variety of facilitators with, as mentioned in the two previous modules, management and co-worker or facility support topping the list $(n=29)$ along with the ability to work together with other nurses to implement communication techniques $(n=5)$. Access to materials and AAC were mentioned often $(n=15)$, as well as support from speech language pathology services $(n=6)$ and clinical instructors or unit educators $(n=6)$.

The implementation of PAS, per participant report, benefited from the low financial cost and easy implementation of the strategy $(n=2)$ and improved understanding of what works best for patients $(n=1)$. Desired or predicted facilitators included accessible education on AAC for nursing students $(n=1)$, a budget for AAC $(n=1)$, AAC supports that can be created for specific units $(n=1)$, time to educate patients and families on PAS (n 


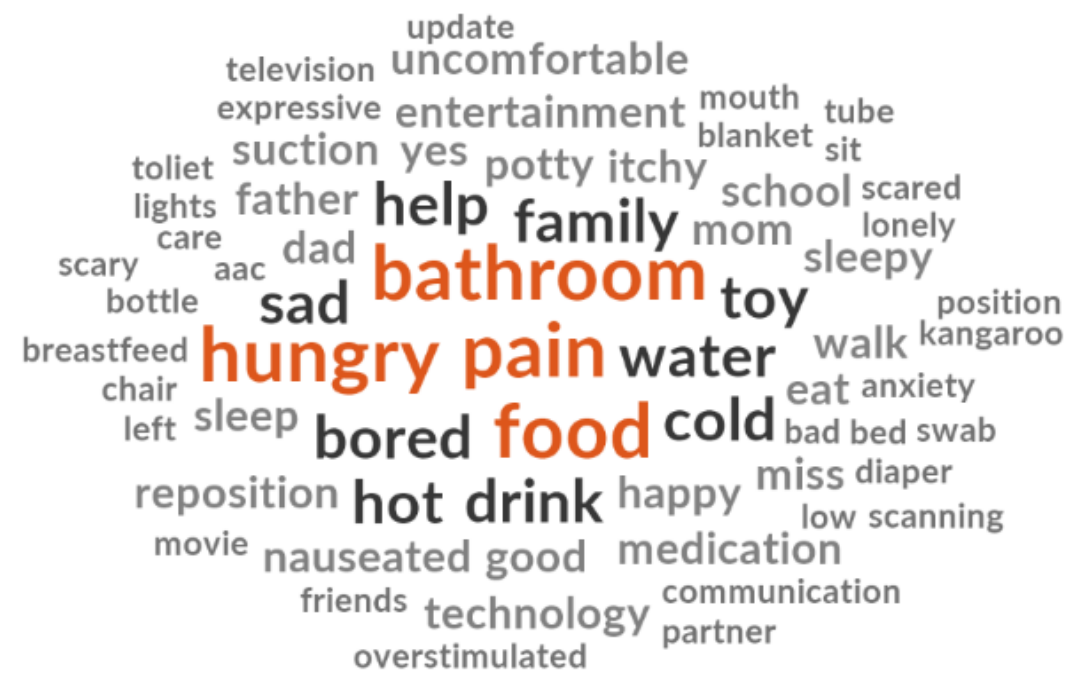

Figure 4.8: Top 5 Vocabulary for a Low-Technology Board.

$=3)$, and additional training $(n=2)$ or the spread of this education $(n=1)$. Participants also reported they could not identify any facilitators $(\mathrm{n}=1)$, the information did not apply to their patient population $(n=1)$, or they were unsure of potential facilitators $(n=1)$.

One participant requested more information on sign language. Overall, participants reported no additional questions or information requests $(n=20)$ and messages of thanks for the training $(n=5)$.

Following the completion of the module 3 post-test, a few additional questions were posed to gain an understanding of participant access to low-tech AAC, need for low-tech $\mathrm{AAC}$, understanding and use of the referral process, and vocabulary they feel is of highest priority for their patients.

Participants were asked, if they were to create a low-tech AAC board, what the top five vocabulary words were that they would include for their patient population and work setting. The most requested terms were food/eat/hungry $(n=56)$, bathroom/potty/toilet $(\mathrm{n}=48)$, pain/hurt/hurting $(\mathrm{n}=47)$, drink/thirsty $(\mathrm{n}=20)$, water $(\mathrm{n}=19)$, entertainment/movie/TV $(\mathrm{n}=11)$, and family $(\mathrm{n}=11)$. Additional commonly requested terms included toys/play $(n=7)$, sleep $(y) /$ nap/tired $(n=7)$, cold $(n=7)$, sad $(n=7)$, mom $/$ mother $(n=6)$, hot $(n=6)$, help $(n=6)$, dad / father $(n=5)$, happy $(n=5)$, and bored $(n=5)$. A word cloud depicting the vocabulary provided by participants can be found in Figure 4.8.

Participants provided a range of patients they see each week who might benefit from low-technology AAC (see Table 4.6). Just over $72 \%$ of participants reported seeing 1-4 patients who would benefit from low-technology AAC in a week if they had access to it. 
Table 4.6: Patients Who Would Benefit from Low-Technology AAC per Week.

\begin{tabular}{lcc}
\hline Number of Patients & Frequency & Percent \\
\hline $\mathbf{0}$ & 4 & $5.8 \%$ \\
$\mathbf{1 - 2}$ & 24 & $34.8 \%$ \\
$\mathbf{3 - 4}$ & 26 & $37.7 \%$ \\
$\mathbf{5 - 6}$ & 5 & $7.2 \%$ \\
$\mathbf{7 - 8}$ & 4 & $5.8 \%$ \\
$\mathbf{8 +}$ & 6 & $8.7 \%$ \\
TOTAL & 69 & $100 \%$ \\
\hline
\end{tabular}

Participants were asked whether they have access to low-tech AAC at their facility. Ten participants reported they do have access, while 12 reported no access, and an additional 47 participants did not respond to this question. This is likely because 44 participants are nursing students who may not have felt able to answer this question. An additional question queried whether participants felt they could access low-tech AAC during their next shift with 12 responding 'yes', 15 responding 'maybe', and an additional 30 responding 'I don't know'. There were 12 missing responses for this question.

Additional questions probed who provided low-tech AAC in their facility with a variety of answers provided. Seventeen participants reported that SLPs provided the low-tech AAC supports in their facility, while an additional 12 stated it is Child Life at their hospital, and four stated nursing provided low-tech AAC. An additional 12 were missing and 22 responded 'other'. Eighteen participants provided open-ended responses indicating they were 'unsure' $(\mathrm{n}=13)$, a behavior analyst would provide low-tech AAC $(n=1)$, do not have low-tech AAC $(n=1)$, probably Child Life or SLP $(n=1)$, or indicating they are a nursing student $(\mathrm{n}=2)$.

Most participants reported referring to speech-language pathology infrequently $(<1$ time per month; $n=25,36.2 \%)$. An additional 21.7\% $(n=15)$ reported referring to speech pathology 1-2 times per month and 12 more reporting 3-4+ per month (17.4\%). Eleven participants chose to provide a written response which indicated they are a student $(\mathrm{n}=$ $7)$, or they had never referred to speech language pathology $(n=3)$, and one participant stated it is out of their scope of practice. Thirty-eight participants (55.1\%) reported a desire for additional information on other types of AAC. Sixteen of the 69 participants $(23.2 \%)$ reported a desire to watch a fourth optional module. One nursing student participant chose to create and upload a low-tech communication board that can be seen in Figure 4.8.

The sum total of this qualitative and quantitative data indicate that this education was timely and pertinent, proving to effectively provide information in a manner that was 
digestible and beneficial to nurses and nursing students, and will hopefully prove beneficial to their patients as well. 


\section{Chapter 5}

\section{Discussion and Conclusions}

\subsection{Qualitative Discussion}

RI-1. What content do pediatric nurses and nursing students find relevant to their clinical work?

\subsubsection{Pediatric Nurse Qualitative Data}

Pediatric nurse participants provided a wealth of knowledge and experience through their interview and focus group discussions. The 10 pediatric nurse participants reported that communication was relevant to their clinical work. They felt there was an expectation that they communicate effectively with their pediatric patients, though they did not always feel they had the tools, training, and resources to do so. An additional sub-theme emerged during the pediatric nurse interviews and focus groups not seen during the nursing student discussions on the importance of communication. This theme highlighted their personal priorities of effective communication and their experiences when it was lacking. This likely emerged with this group and not with nursing students, as these seasoned pediatric nurses had felt the impact of communication breakdowns in their clinical experience, with the full weight and responsibility that nursing students are often shielded from in clinical rotations.

Pediatric nurses were familiar with some of the terms (ex., effective communication) and communication strategies (ex., facing the communication partner, using pen and paper), though they often reported a lack of consistent access to tools and communication supports (ex., low-technology communication boards, in-person interpreters, picture supports) as well as barriers and constraints such as time, language barriers, and lack of knowledge or experience with communication supports. Nurse participants did report a strong multidisciplinary team atmosphere at their facilities that they could draw from as well as the potential benefit of efficient communication (i.e., less time to communicate) if AAC were understood and implemented often.

A variety of patient populations were identified through the focus group and interview discussions who might benefit from AAC supports and strategies. During the discussions, nurses began with commonly identified populations such as patients who are 
hard of hearing, English as a second language, or non-verbal autism; however, as discussions continued, nurses identified a growing list of potential beneficiaries such as children who are fearful or shy, patients post T\&A, or toddlers who often experience larger emotions and thoughts than they have language and vocabulary to support in hard medical care situations. Thus, nurses appeared to see the potential benefit for many pediatric patients, populations, and situations during our discussions.

The pediatric nurse participants came from a variety of backgrounds and training experiences with most holding a bachelor's or master's degree. They were in consensus regarding important aspects of continuing education, including that they be short, engaging, and clinically relevant, including case studies or vignettes if possible. All of these aspects were incorporated into the development of the CBLs in this research. Nurse participants overall felt that additional education on communication supports and AAC was much needed. The majority of this sample of participants reported a desire for more information on low-technology AAC ( $\mathrm{n}=4)$ as well as information on obtaining AAC and how best to support patient communication through a strong interdisciplinary approach. Participants likely requested additional information on low-technology AAC supports as they are versatile, low-cost, and can be easily created, maintained, and replicated.

An additional education sub-theme on patient education emerged specifically with the pediatric nurse discussions, likely due to the greater clinical experience of practicing nurses. In contrast, this sub-theme may not have emerged with nursing students who have a variety of short clinical rotations during their programs to meet certain training and education requirements. These short clinical experiences will not provide the full experience nor exposure to all of the roles and responsibilities of a full-time nurse, such as discharge education experiences or interacting with families and loved ones during hard decisionmaking situations. Pediatric nurses reported a variety of patient education experiences, highlighting the importance of patient and family education and the role communication plays during those interactions. They often mentioned the use of strategies to help teach new information to patients and families such as learn one, do one, teach one, and teach back strategies. Handouts or a notebook for families to take home after a hospital admission were mentioned multiple times as a support for families. This additional sub-theme highlights the importance of communication strategies and the breadth of communication opportunities nurses experience in their clinical work.

Pediatric nurse participants voiced an understanding of the important role that communication plays in a pediatric hospital admission or medical interaction. They were able to provide some examples of communication supports and strategies, though communication breakdowns and their subsequent implications were common to their experiences. Time, access to communication supports, and knowledge appeared to be three primary barriers to effective communication, two of which this research seeks to address (i.e., access and knowledge). Nurses requested education that was clinically applicable, short with the option of returning to it if needed, and engaging. Nurses stated their perceived need for additional education and training in the area of communication supports to provide better 
patient care and do their jobs to the best of their ability. This research seeks to meet that request for additional education to prepare nurses and nursing students to provide the highest quality care for patients, ensuring effective communication.

\subsubsection{Nursing Student Qualitative Data}

Nursing students were aware of the importance of communication and its potential role in their future clinical work, regardless of their area of interest (ex., geriatrics, mental health, pediatrics, etc.). The students reported a heightened awareness and sensitivity to cultural, linguistic, and demographic differences than seen in the pediatric nurse discussions. This may be due to course content and recent current events which have highlighted the necessity for sensitivity in clinical and medical settings for patients from all cultures, backgrounds, and languages.

There was little difference between the nursing student and pediatric nurse understanding of terminology pertinent to the communication discussion. Both groups were overall unfamiliar with the acronym AAC, the terms expressive and receptive language, and complex communication needs or communication vulnerability. The nursing students were unaware and had very little experience with communication supports or AAC of any kind other than the pain scale. This differed from the pediatric nurses who were able to provide some examples of no-tech and low-tech strategies, often as a part of a recollection of a particular patient; however, the nursing students appeared more cognizant of therapeutic communication and its role in their clinical work. Therapeutic communication, defined by The National Commission on Correctional Health Care (2011) as "the face-to-face process of interacting that focuses on advancing the physical and emotional well-being of a patient," plays a role in effective communication. However, it does not consider the necessary inclusion of comprehending and expressing for the goal of information exchange. AAC supports and strategies assist in rounding out this assurance of comprehensive communication between both parties to ensure that patients feel safe and at ease as well as ensuring they are heard, understood, and comprehend. This research has sought to round out the picture of communication to include the personal aspect identified and targeted with therapeutic communication and to address the importance of information exchange to achieve true effective communication.

Most nursing students were not familiar with communication supports or strategies outside of a pain scale. Nursing students were engaged and excited to learn about the variety of AAC supports and strategies that exist. One participant had clinical experience using AAC, with only two nursing students having even seen a low-tech communication board used (one in a skilled nursing facility and another while working overseas). Despite limited clinical experience, student participants were more vocal regarding ideas for ways to adapt a hospital room to include AAC or things that might be changed to better support communication. Their ideas included using the TVs in patients rooms for AAC access or education as they are already setup for patient education modules. Another student suggested we consider attaching large AAC boards to the white boards and provide patients 
with a laser pointer to select and communicate information. These ideas demonstrated a level of interest, creativity, and engagement that is encouraging to see in this new generation of nurses.

Nursing students initially identified primarily adult populations such as patients post-stroke, patients with dementia, individuals who are deaf or hard of hearing, individuals with visual impairment. Few nursing students had completed their pediatric clinical rotation, thus for many of them adult populations were at the forefront of their minds. As with the pediatric nurses, as discussions continued, students thought of more populations who could benefit from communication supports such as patients on the renal floor with increased confusion, individuals who are English as a second language, or children who have autism and are limited verbal.

Nursing students found it harder to identify barriers and facilitators, likely due to the nature of clinical rotations which are short and include a variety of experiences. Nursing students provided more general potential barriers and facilitators such as time, staffing, access, and knowledge. The nursing students also mentioned a potential barrier they could foresee in seasoned nurses being less willing to learn new information or change their habits. This is likely something they have glimpsed in clinical rotations, working directly under a preceptor. This is an interesting observation and reported barrier which points to a potential need to provide information, as done in this research, to nursing students as well as practicing nurses. It may be that following education, practicing nurses are less likely to change their behavior than nursing students as more seasoned nurses have their way of doing things, while nursing students are still developing those skills, habits, and procedures. This research sought to provide succinct yet high-quality education on AAC to students, in particular, in order to prepare them for their clinical work, in hopes that they will have a foundation of knowledge and enlist these communication strategies and supports as they begin their nursing careers. Students also mentioned that the ease of using the no-tech, low-tech, and mid-tech AAC was a facilitator. They also mentioned a possible facilitator in the education and inclusion of CNAs on AAC as they spend more time with and provide more one on one care to patients.

Nursing students reported on a variety of educational and clinical experiences, with each school and program being slightly different in organization and content. Students reported minimal education in the classroom or lab setting related to communication supports outside of the emphasis on therapeutic communication. They felt that alternative communication could be helpful in their clinical career and reported a desire to learn more about it. Responses and opinions were mixed regarding the type of AAC that they felt would be most beneficial to learn more about: low-technology AAC ( $n=2)$, midtechnology AAC $(n=2)$, and high-technology AAC $(n=1)$. The focus group discussion with two nursing students included a difference of opinion with one student desiring more information on high-tech AAC because of its versatility and adaptability, and the other student requesting more information on low-tech AAC such as communication boards for the ease of implementation across a variety of patient populations with a lower cognitive 
demand than high-tech AAC. This discussion highlights the differing of opinions as well as the importance and need for more education on AAC of all kinds, as there is no one size fits all or silver bullet. Students reported a willingness to invest more time on education modules than pediatric nurses, likely due to their current career as a student. They also reported that they felt a majority of their classmates would be interested in learning more about this information as they considered it highly clinically applicable and beneficial.

Nursing students were excited about this information and reported the perceived benefit that AAC could have in a clinical setting. Though they were all still in short clinical rotations, with no 'home base' per se to think about direct AAC implementation, they had a variety of clinical rotation experiences to reflect on. They provided a wide variety of patient populations who might benefit from AAC, children through adults, as well as a similar list of proposed barriers and facilitators that they might encounter. Their enthusiasm to think through problem-solving ideas for innovative yet simple AAC 'hacks' speaks to their desire to provide holistic patient care through the lens of therapeutic communication with a new shifted focus on effective communication.

This information was analyzed and used to create three 15-minute online education modules on no-tech and low-tech AAC to strive to remove some of the knowledge barriers reported by both nurses and students. Themes and sub-themes were targeted and embedded in the modules and recommendations regarding the organization of the CBLs was taken into consideration. Participants varied in their preference for additional information regarding no-tech, low-tech, mid-tech, and high-tech AAC; however, it was determined that no-tech and low-tech AAC methods, often requested, would be easy to implement, easily accessible, and affordable to obtain and maintain. Participants were provided with the opportunity to receive two low-tech double-sided communication boards to provide access for clinical use. Following their participation, all participants received a digital synopsis of each education module via email along with links to the provided free low-tech communication boards and a variety of other freely available communication supports. Knowledge and access are two barriers which we feel we have begun to address in this research endeavor.

\subsection{Quantitative Discussion}

Participants were provided a pre-test focused on knowledge and self-efficacy followed by a short CBL and the same knowledge and self-efficacy questions. The post-test also included five Likert scales to measure learning self-efficacy which included cognitive, affective, and psychomotor scales, and scales measuring the perceived relevance of the content, and effectiveness of the educator and education method.

RI-2. Based on pre- and post-test results from the computer-based learning modules, how do pediatric nurses and nursing students':

a. Knowledge of AAC change 


\section{b. Self-efficacy using AAC change}

The results of the repeated measures ANOVA indicated a statistically significant change from pre- to post-test for all three modules with modules 1 and 2 demonstrating no difference between pediatric nurses and nursing students. For both modules 1 and 2 nurses and students had a significant increase in knowledge score from pre- to post-test indicating they learned new information following the education modules. Module 1 pre-test average of $5.45(54.50 \%)$ increased to 9.55 (95.50\%) at post-test which is a notable increase in knowledge score. Pre-test average for Module 2 of $12.52(83.47 \%)$ increased at post-test to $13.91(92.73 \%)$ which is smaller though still statistically significant $(p<.001)$ benefit.

For the Module 3 knowledge outcome an interaction effect was found. Further investigation with the use of independent samples t-tests indicated that pediatric nurses scored lower on the pre-test than nursing students, however, post-test results were not significantly different. This indicated that nursing students had a higher pre-test score than nurses, but following the education, both groups had approximately equivalent knowledge scores. According to this research, pediatric nurses learned at a greater rate than nursing students, however, knowledge outcomes at post-test were approximately the same $(95.80 \%$ accurate). This indicates that both groups were able to learn the new information covered in the CBLs.

All three modules indicated post-test scores greater than $92 \%$ indicating content mastery. This would indicate that this method of education could benefit practicing nurses, as we often see continuing education presented in this manner, as well as nursing students, most of whom have interacted with online teaching especially throughout the global COVID-19 pandemic. This indicates that nurses and nursing students may still benefit from information on no-tech and low-tech AAC, often a hands-on tool, as they form the foundation of knowledge needed to implement AAC strategies in practice moving forward.

Self-efficacy, the perception of our ability to do something, enlist a skill, or complete a task, was measured using a $100 \mathrm{~cm}$ visual analog slider scale in the pre- and post-test of each module. Smaller sample sizes are seen with this measure which may be due to its location at the end of each test (pre and post), the requirement of moving the slider to register a response (if they felt their confidence was at 50 - where the slider was originally, they must move it to register a score), or a lack of understanding of the concept of self-efficacy.

A repeated measures ANOVA was run for module 1, indicating a statistically significant increase in self-efficacy from pre- to post-test. Nurses reported a slightly higher (pre-test $M=52.00$ ) and statistically significant $\left(F(1,62)=5.82, p=.02, \eta^{2}=.09\right.$ ) self-efficacy rating than nursing students (pre-test $M=38.47$ ). This follows logic that practicing nurses have more confidence using communication strategies than students who are still learning and developing their clinical skills. However, following their participation in the module 1 CBL, all participants significantly increased their self-efficacy scores at a similar rate (pre: $M=42.91$, post: $M=83.48$ ). 
An interaction effect was found for self-efficacy in module 2 and module 3. Independent samples t-tests delved deeper into the relationship between group and time for each of these modules. Module 2 results indicated a statistically significant difference between pediatric nurses and nursing students at the pre-test (student $M=33.30$, nurse $M=63.10$ ), but found that both groups rated their self-efficacy approximately the same at the post-test (student $M=79.67$, nurse $M=84.08$ ).

Module 3 results mimicked module 2 with an interaction effect found, resulting in further analysis using independent samples t-tests. These tests indicated a statistically significant difference at pre-test with nurses indicating a higher self-efficacy than students (student $M=20.28$, nurse $M=37.00$ ). Post-test results indicated both groups rated their self-efficacy approximately the same (student: $M=83.23$, nurse: $M=87.00$ ).

The analyses for each of these modules indicates that pediatric nurses and nursing students grew in their perceived self-efficacy when provided with specific education. Following the education, both groups indicated confidence in their ability to achieve a desired outcome, in this case using no-tech and low-tech strategies such as wait time, simple sign language, and partner-assisted scanning, which was markedly higher than their pre-test measure. Thus, it can be assumed that the information provided increased their perceived ability to utilize communication supports in their clinical work.

This measure provided a measure of attitude and confidence, both of which are intangible yet essential when considering behavior change. The measurement of behavior change is outside of the scope of this research; however, the self-efficacy measure from pre-test to post-test provides a picture of nurse confidence in implementing AAC strategies in their clinical work. Additional research in this area to determine whether behavior change follows increased self-efficacy should be considered.

RI-3. Based on post-assessment results from the CBLs, how do pediatric nurses and nursing students rate the:
a. Adapted Learning Self-Efficacy Scale (Cognitive, Affective, Psychomotor)
b. Relevance of Content
c. Effectiveness of the Educator and Method

All five scales were assessed for reliability using Cronbach's alpha, with acceptable reliability measured at $\alpha \geq .70$. The affective scale was adapted in all three modules, as modules 1 and 2 reliability measures were inadequate without the removal of the initial item. This item was removed from the module 3 affective scale for consistency. The psychomotor scale in module 1 was also adapted as discussed in Chapter 4 . The cognitive, relevance, and effectiveness scales all demonstrated adequate reliability with the inclusion of all items for all three modules.

A MANOVA was utilized to determine scale significance while reducing Type I error. The module 1 MANOVA did not result in a statistically significant result; however, 
modules 2 and 3 did, resulting in further assessment of the one-way ANOVAs for each scale. The relevance scale was statistically significant for modules 2 and 3 based upon group. Both module 2 and 3 indicated nursing students (module 2: $M=4.56$; module 3: $M$ $=4.44$ ) reported a significantly higher perception of content relevance than pediatric nurses (module 2: $M=4.02$; module 3: $M=3.91$ ). The overall rating indicated that all participants somewhat agreed to strongly agreed with the relevance of the content in each module to a varying degree based upon group membership. These results may be explained by nursing students feeling that education and learning is a larger part of their 'job' right now as they are in school or may be due to an inaccurate understanding of the relevance of this content for their future clinical endeavors. Pediatric nurses may feel that this information is relevant though not as relevant as students because they do not see as great a need for it in their clinical work or because they are already familiar with and implementing some of these communication strategies. It should be noted that both groups indicated somewhat to strong agreement regarding the relevance of the content.

An additional yet difference scale in module 2 and 3 demonstrated a statistically significant difference based upon group. The effectiveness scale for module 2 required further assessment. It was found that based upon this data, nursing students $(M=4.73)$, similar to the relevance scale, found the education mildly more effective than pediatric nurses $(M=4.48)$. This indicates that both groups strongly agreed that the CBLs were effective at communicating information related to choice making and simple sign language. Students may have a slightly higher score on this scale because they are more familiar with online education than pediatric nurses.

The MANOVA for module 3 indicated a significant difference between nurses and students related to the psychomotor scale. This scale was a measure of how comfortable participants felt carrying out actions described and taught in the education. Nursing students $(M=4.61)$ were slightly more confident in their ability to carryout the steps of partner-assisted scanning, per this scale's results, than pediatric nurses $(M=4.49)$, though both averages indicated they strongly agreed with the included items. Nursing students may have an overall higher rating on this scale when compared to nurses because they may perceive that they will be able to easily implement partner-assisted scanning into their clinical work, while practicing nurses are aware of the potential barriers and difficulties associated with implementation of new strategies in clinical acute care.

Four of the five scales achieved the hypothesized average of $M \geq 4$ indicating an average of somewhat to strongly agree. The only scale with averages below 4 (ranging from 3.45 to 3.70) across the three modules was the affective scale of the L-SES. This may have been due to difficult interpretation of the language in the scale. This scale asked you to "compare this education module to other continuing education you have completed." For students, this may have been impossible to do as they may have never had to complete continuing education before. Additionally, some open-ended responses indicated they interpreted the scale to mean they should compare these modules to one another, which 
would have been difficult to do after completing only module 1 . This proposal is supported by the increased reliability found in module $3(\alpha=.88)$.

The validated L-SES scale was created by Kang et al. (2019) as a universal scale to assess learning self-efficacy for clinical skills with medical students initially. Kang et al. (2019) created the scale to be adapted and used with a variety of clinical providers. It is unclear if this scale was originally created and validated in English which could be a limitation to its use and applicability. The cognitive and psychomotor scales demonstrated good reliability and, for this research, their statements were strongly agreed upon by participants. The affective scale, for the above mentioned potential reasons, demonstrated lower reliability and agreement by participants.

These scales provided a reliable assessment of the cognitive (knowledge), psychomotor (perceived ability to use the knowledge and complete an action), relevance (pertinence of the content), and effectiveness of the education and educator all demonstrating positive agreement. The affective scale of the L-SES was the only scale which may require some clarification prior to clinical application. These scales were supported by the open-ended responses at the end of each module post-test.

Participants reported a variety of planned changes to their clinical practice following this education with the most common being awareness of the importance of communication and the use of the strategies discussed in each module (ex., wait time, simple sign language, partner-assisted scanning). Participants stated they hoped that patients would feel that communication interactions were improved by patients feeling more autonomous in their healthcare journey and decision-making processes.

A variety of barriers were listed which echoed barriers mentioned in the qualitative data collection portion of this research. Commonly mentioned barriers included time, access to materials and supports, and knowledge and training. This research hopes to address two of the three primary barriers mentioned as participants were provided with communication boards (access barrier) and education (knowledge barrier). These barriers are reported in the literature not only by healthcare professionals, but by individuals with CCN who report that a lack of availability or provision of AAC or a lack of awareness or knowledge of AAC are barriers to their communication (Moorcroft, Scarinci, and Meyer, 2019). The knowledge barrier was assessed with the pre- and post-test measures in this research and indicated participants learned new information. This result paired with the improved self-efficacy is promising for future implementation of these strategies in their clinical work. The access barrier was not assessed, however, all participants were sent low-technology communication boards who requested them. In addition, all participants were provided with digital links to free online AAC resources and low-tech communication boards. Some participants responded to open-ended questions with gratitude and excitement about using the low-tech communication boards and resources provided via email and posted mail following their participation. 
Facilitators that were mentioned by participants appeared to be both current and desired supports. The most common facilitator mentioned was the need for support from their facility, administration, unit, educators, and co-workers. This support appeared to provide participants with a sense of camaraderie and encouragement to support patient's communication even if it required a learning curve, taking more time initially, and required they try something new. Access to materials and other experts such as SLPs was mentioned as a facilitator. It appeared that this was an experienced benefit to implementing communication supports for some participants and a desired support for others. As with the previously mentioned barriers, the literature reports similar patient perception of the facilitators found in this research, with the reported importance of an interdisciplinary team approach and support from the facility, administrators, and co-workers, as well as access to knowledge, experience, and AAC materials (Finke, Light, and Kitko, 2008; Moorcroft, Scarinci, and Meyer, 2019) being paramount.

Additional comments and questions included requests for a synopsis of the information covered in each module, additional resources, and positive comments regarding the overall experience. All participants were provided with a synopsis of each module and additional resources via email after the completion of all modules. The positive comments indicated a number of participants felt the education provided them with new and useful information that would benefit their patients and their clinical practice.

\subsection{Study Limitations}

This research was not without limitations. A limitation may be found in the mostly online data collection process due to the COVID-19 pandemic which began approximately one month into qualitative data collection. Plans were in place from the outset of data collection to include VOIP data collection in order to include participants from across the country, thus a large shift was not required. However, the pandemic prevented additional in-person data collection and likely impacted the number of focus groups that could be conducted.

Selection bias is always a limitation consideration with qualitative and survey research. Participant recruitment was conducted through social media, posted flyers, and reaching out to 36 universities, 25 children's hospitals, 31 pediatric nurse and student organizations and 10 groups on social media. Some participants may have participated following the spread of information via word of mouth from participant to participant, though this was not directly tracked. Despite a wide net being cast for participant recruitment, individuals were able to self-select into the research, which may indicate a bias toward common research participation or particular interest in the topic of communication among other things.

Another limitation may be found in the unequal group sizes (pediatric nurses $=25$, nursing students $=44$ ). Participants from the same nursing program or hospital may have gone through the education modules together which could be a limitation that may have skewed pre-test or post-test scores. An additional potential limitation could be related to 
time. Participants could start and stop the education modules as much or as little as they wanted. Some participants completed the modules without stopping as evidenced by the start and end timestamps, however, others completed module content over days and weeks. The retention questions were included in modules 2 and 3 in order to assist in capturing some indication of carryover, with all results indicating regardless of time taken within or between modules, knowledge retention remained.

\subsection{Conclusions}

Overall, the qualitative and quantitative results from this research align to demonstrate that participating pediatric nurses and nursing students felt knowledge of communication and AAC supports were and remain important to their clinical work and field; however, they reported a paucity of resources (educational and physical) available to them. This research supports the continued development and implementation of AAC education through the use of CBLs to meet educational and access barriers mentioned throughout this research and the literature (Finke, Light, and Kitko, 2008; L. Patak et al., 2009; Hemsley and Balandin, 2007; Moorcroft, Scarinci, and Meyer, 2019).

This research sought, in a small way, to meet the voiced needs by nurses and students, while answering the call by Costello, L. Patak, and Pritchard (2010), Finke, Light, and Kitko (2008), Hemsley and Balandin (2007), Moorcroft, Scarinci, and Meyer (2019), and L. Patak et al. (2009) to provide efficient education and research support to determine its effectiveness. Feedback from participants and statistical analyses indicate that these short, 15-minute online education modules were effective at providing information, increasing participants' felt ability to use the provided information, and was perceived to be relevant to their clinical work in healthcare. This research aligns with the mission, mandate, and assurance of ethical healthcare provision laid out by the Joint Commission and World Health Organization which states that healthcare providers must provide an effective method of communication for all patients (The Joint Commission, 2010; World Health Organisation, 2011). Future research will build upon this foundation to provide additional education on AAC, improved access to AAC supports and resources to clinical healthcare professionals on the front lines, and seek to build interdisciplinary teams providing the highest quality healthcare through effective communication. 
Appendix A

Summary of Relevant Research 


\section{Table A.1: Summary of Relevant Research with Nurses and Pediatric} Patients and AAC.

\begin{tabular}{|c|c|c|c|c|c|}
\hline Author & $\begin{array}{c}\text { Title } \\
\end{array}$ & $n$ & Population & Setting & Design \\
\hline Costello (2000) & $\begin{array}{l}\text { AAC intervention in the } \\
\text { intensive care unit: The } \\
\text { children's hospital Boston } \\
\text { model }\end{array}$ & 43 & Pediatric patients & Preop, ICU & $\mathrm{RA}$ \\
\hline Costello et al. (2010) & $\begin{array}{l}\text { Communication vulnerable } \\
\text { patients in the pediatric ICU: } \\
\text { Enhancing care through } \\
\text { augmentative and alternative } \\
\text { communication }\end{array}$ & N/A & Pediatric patients & ICU & BP \\
\hline Hemsley et al. (2013) & $\begin{array}{l}\text { Supporting communication } \\
\text { for children with cerebral } \\
\text { palsy in hospital: Views of } \\
\text { community and hospital staff }\end{array}$ & 49 & $\begin{array}{l}\text { Community and } \\
\text { hospital-based } \\
\text { allied health } \\
\text { professionals } \\
\text { and hospital nurses }\end{array}$ & Hospital & QFGs \\
\hline Mesko et al. (2011) & $\begin{array}{l}\text { Use of picture } \\
\text { communication aids to } \\
\text { assess pain location in } \\
\text { pediatric postoperative } \\
\text { patients }\end{array}$ & 27 & $\begin{array}{l}\text { Pediatric patients } \\
\text { undergoing T\&A }\end{array}$ & PACU & D \\
\hline
\end{tabular}

Summarized Results

provide pediatric patients with AAC support preoperatively - 3 phases.

1.Pre-op: SLP led education on AAC access and techniques. Voice banking and vocabulary selection occurs at this pre-op phase.

2.Post-op: assistance getting AAC setup and implemented in PACU or ICU 3.Discharge interview: Interview with patients, family, healthcare staff. - Patients: felt more independent, less anxious, maintained personality.
- Parents: appreciated preop preparation, comforted by child's ability to communication in their absence, and higher perceived quality of life. - Nurses: better able to form relationships with patients. Patients more efficiently communicated urgent needs.

Three phase model was successful for all parties in preparing for temporary loss of speech, implementing AAC, and better quality of life and patient needs met postoperatively.

3 Phase AAC Implementation in PICU:

Phase 1: emerging from sedation - getting attention and responding to yes/ no questions.

- Access to nurse call system

- Determine reliable yes/no; healthcare staff to offer third option (ex., I don't know).

Phase 2: increased wakefulness - communication base information with staff and family.

- Picture communication boards, alphabet boards, alternative access methods, speech generating devices (SGDs), amplification Phase 3: Need for broad and diverse communication access - communication about and beyond hospital environment.

- Computers and SGDs, assistive and mainstream technologies.

Investigated community and hospital-based allied health and nursing staff communication interactions with pediatrics with cerebral palsy (CP) and CCN - AAC is not widely used, understood, or accepted by nurses due to time constraints, lack of storage, difficulty mounting and using at bedside, lack of knowledge or experience, and preference to communicate with parents - No perceived need for kids with CP to communicate more than immediate wants and needs

Some participants requested access to low-tech AAC. Most were hesitant to try high-tech AAC. Need for nurse buy-in prior to AAC education and training to support AAC needs of $\mathrm{CP}$ patients with $\mathrm{CCN}$.

Pain assessment was measured by nurses (normal method) and researchers (use of AAC picture communication aids) to determine patient pain location post-surgery.

-118 measures were made across 27 patients with nurses providing no pain location identified with $32 \%(\mathrm{n}=38)$. - Pain location documented by PACU nurse agreed with child using pictures $54 \%(\mathrm{n}=64)$.

$-38 \%(n=45)$ PACU nurse pain location did not agree with child's location of pain.

-28 times nurse documented child's pain at surgery site, but child indicated a different location.

- Parent satisfaction measure indicated high satisfaction with the use of picture supports.

- Recommend additional study - pain assessment in intensity and location is important during hospitalization - need to develop consistent and reliable pain assessment tools for nurse use with pediatrics.

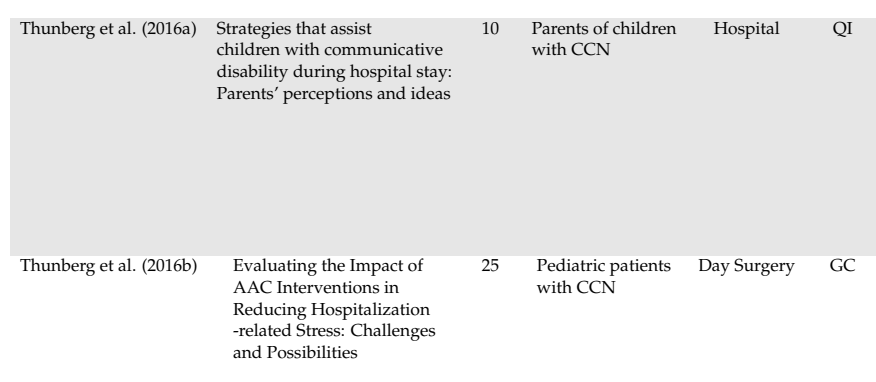

Parents reported:

- Importance of communication and understanding between child and staff. o Staff should seek to meet the child where they are and adjust to their communication needs.

- mportance of knowledge and skills in AAC and special needs.** o Staff should know about AAC and be able to use it with all patients Need for individualized care.

o Staff should seek to meet patient and family's needs - context specific. Perceived safety due to interaction and environment. o Strive for staff continuity and helping child feel comfortable while at the hospital.

Saliva cortisol levels (indicate anxiety) measured in 15/25 kids during daysurgery experience - children with CCN had higher means levels of cortisol indicating higher anxiety pre-surgery.

- AAC (picture schedule) provided to most participants - Participants with AAC schedule required little to no pre-medication prior to surgery.

- State-Trait Anxiety Inventory State (STAI-S) given to parents and short STAI given to children - both demonstrated decreased anxiety post-surgery - Children with CCN had slightly higher anxiety level prior to surgery compared to typically developing children (Nilsson et al., 2012). - More research needs to be done with larger sample size, development an developing) benefited from AAC use in this study. 


\section{Appendix B}

\section{Pre-Test and Post-Test Questions}

\section{Demographic Information}

1. Are you currently a student?

o Yes

o No

Skip To: Q7 If Are you currently a student? = No

2. What degree are you working towards?
o BSN
o ABSN
o $\mathrm{RN}$ to $\mathrm{BSN}$
o MSN
o DNP
o $\mathrm{PhD}$
o Other

3. What school are you attending?

4. Are you a currently practicing healthcare professional?
o Yes
o No

Skip To: Q11 If Are you a currently practicing healthcare professional? = No

5. In what capacity are you practicing?

o CNA 

o Nurse
o Nurse Practitioner
o Other

6. Which department/unit do you work in?
o ED/ER
o PICU
o Medical-Surgical
o Oncology
o NICU
o Neurology
o Outpatient
o Pulmonology
o Renal
o Cardiology
o Rehab
o Multiple/Floater
o Other

7. What shift do you typically work?
o Day
o Night
o Evening
o Other

8. What is your employment status?
o Full time
o Part time
o PRN
o Other

9. Do you have any current certifications/credentials?

o Yes 
o No

Skip To: Q103 If Do you have any current certifications/credentials? = No

10. What are your current certifications/credentials?

11. What region of the US do you work in?
o Northeast
o Southeast
o Midwest
o Southwest
o West
o Other

INSTRUCTIONS: This research includes 3 educational modules with pre-test questions, a short 15 minute educational video on communication supports, and post-test questions. The post-test for each module is longer than the pre-test. You may start and stop at any time throughout these modules; however, we would HIGHLY suggest you complete each module in its entirety (pre-test, video, post-test) in one sitting if possible - aka take breaks between modules if you can. Your personalized link will allow you to start, stop, and return to your educational modules by simply clicking on the link again to pick up where you left off. Thank you for your participation!

\section{MODULE 1: Wait Time, Rephasing/Recasting Pre-Test}

1. What are the two parts of communication (choose two)?

o Expressive Communication

o Quiet Communication

o Receptive Communication

o Functional Communication

2. What does AAC stand for?

3. What are two examples of no-technology communication strategies (choose two)?

o Wait Time

o Patience

o Recasting 
o Providing cues

o Finishing their sentences

4. List or describe two pediatric populations/diagnoses/situations that could benefit from communication strategies.

5. What does OWL stand for?

o Object, Witness, Listen

o Observe, Wait, Listen

o Observe, Witness, Lesson

o Object, Wait, Lesson

6. Self-efficacy is defined as a belief in one's capabilities to overcome the demands of a situation in order to achieve a desired outcome. Please move the slider on the scale to indicate your level of self-efficacy regarding your ability to use notechnology AAC.

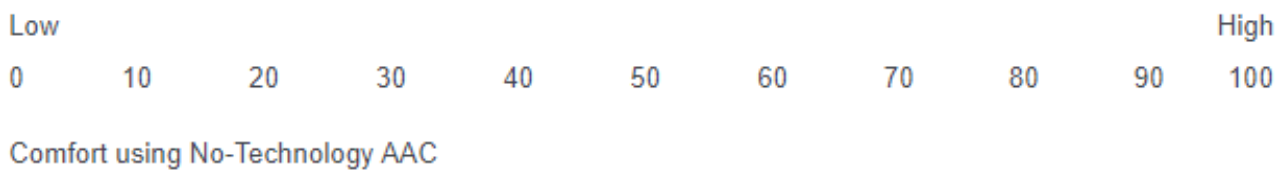

\section{Module 1: Wait Time, Rephrasing/Recasting Post-Test}

1. What are the two parts of communication (choose two)?

o Expressive Communication

o Quiet Communication

o Receptive Communication

o Functional Communication

2. What does AAC stand for?

3. What are two examples of no-technology communication strategies (choose two)?

o Wait Time

o Patience

o Recasting 
o Providing cues

o Finishing their sentences

4. List or describe two pediatric populations/diagnoses/situations that could benefit from communication strategies.

5. What does OWL stand for?

o Object, Witness, Listen

o Observe, Wait, Listen

o Observe, Witness, Lesson

o Object, Wait, Lesson

6. Self-efficacy is defined as a belief in one's capabilities to overcome the demands of a situation in order to achieve a desired outcome. Please move the slider on the scale to indicate your level of self-efficacy regarding your ability to use notechnology AAC.

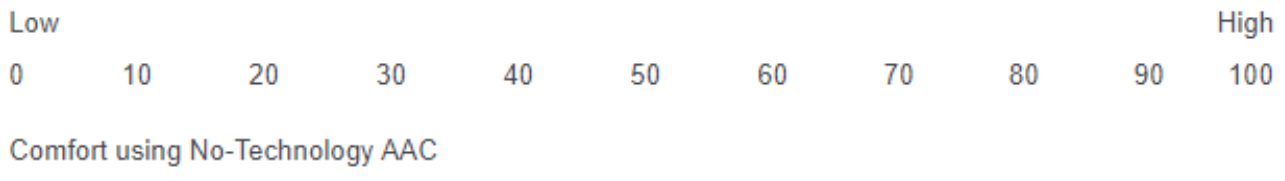

Table B.1: Module 1: Cognitive L-SES

\begin{tabular}{|c|c|c|c|c|c|}
\hline & $\begin{array}{l}\text { Strongly } \\
\text { disagree }\end{array}$ & $\begin{array}{l}\text { Somewhat } \\
\text { disagree }\end{array}$ & $\begin{array}{l}\text { Neither agree } \\
\text { nor disagree }\end{array}$ & $\begin{array}{l}\text { Somewhat } \\
\text { agree }\end{array}$ & $\begin{array}{l}\text { Strongly } \\
\text { agree }\end{array}$ \\
\hline I can recall how to perform wait time. & $\mathrm{o}$ & o & $\mathrm{o}$ & $\mathrm{o}$ & o \\
\hline $\begin{array}{l}\text { I understand the content of rephrasing } \\
\text { and recasting and can demonstrate it to others. }\end{array}$ & $\mathrm{o}$ & o & $\mathrm{o}$ & $\mathrm{o}$ & o \\
\hline $\begin{array}{l}\text { I can verbally explain the purpose and } \\
\text { principle of using no-technology AAC. }\end{array}$ & o & o & $\mathrm{o}$ & $\mathrm{o}$ & $\mathrm{o}$ \\
\hline $\begin{array}{l}\text { I can verbally explain the sequence and } \\
\text { interrelationship between each step. }\end{array}$ & $\mathrm{o}$ & $\mathrm{o}$ & $\mathrm{o}$ & $\mathrm{o}$ & o \\
\hline
\end{tabular}

12. What one change will you make in your clinical practice as a result of what you learned in this module?

13. How will your patients benefit from what you have learned?

14. What barriers might make it difficult for you to implement what you have learned (ex. no access to AAC resources)?

15. What facilitators might make it easy to implement what you have learned (ex. support from management to implement communication supports)? 
Table B.2: Module 1: Affective L-SES

\begin{tabular}{|c|c|c|c|c|c|}
\hline & $\begin{array}{l}\text { Strongly } \\
\text { disagree }\end{array}$ & $\begin{array}{l}\text { Somewhat } \\
\text { disagree }\end{array}$ & $\begin{array}{l}\text { Neither agree } \\
\text { nor disagree }\end{array}$ & $\begin{array}{l}\text { Somewhat } \\
\text { agree }\end{array}$ & $\begin{array}{l}\text { Strongly } \\
\text { agree }\end{array}$ \\
\hline $\begin{array}{l}\text { I think I spent more time on this module } \\
\text { than on others. }\end{array}$ & $\mathrm{O}$ & $\mathrm{O}$ & $\mathrm{O}$ & o & $\mathrm{O}$ \\
\hline $\begin{array}{l}\text { I think I gained more from this module } \\
\text { than others. }\end{array}$ & $\mathrm{O}$ & o & $\mathrm{o}$ & $\mathrm{O}$ & $\mathrm{O}$ \\
\hline $\begin{array}{l}\text { I tend to pay more attention to } \\
\text { information related to this module. }\end{array}$ & $\mathrm{O}$ & o & $\mathrm{o}$ & $\mathrm{o}$ & $\mathrm{O}$ \\
\hline $\begin{array}{l}\text { I tend to actively look for information } \\
\text { related to this module. }\end{array}$ & $\mathrm{O}$ & $\mathrm{O}$ & o & $\mathrm{O}$ & $\mathrm{O}$ \\
\hline
\end{tabular}

Table B.3: Module 1: Psychomotor L-SES

\begin{tabular}{|c|c|c|c|c|c|}
\hline & $\begin{array}{l}\text { Strongly } \\
\text { disagree }\end{array}$ & $\begin{array}{l}\text { Somewhat } \\
\text { disagree }\end{array}$ & $\begin{array}{l}\text { Neither agree } \\
\text { nor disagree }\end{array}$ & $\begin{array}{l}\text { Somewhat } \\
\text { agree }\end{array}$ & $\begin{array}{l}\text { Strongly } \\
\text { agree }\end{array}$ \\
\hline $\begin{array}{l}\text { I can precisely imitate the instructor's steps } \\
\text { and actions of wait time. }\end{array}$ & $\mathrm{O}$ & $\mathrm{o}$ & 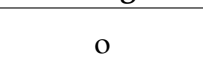 & o & o \\
\hline $\begin{array}{l}\text { I can smoothly complete the operational } \\
\text { steps of rephrasing and recasting. }\end{array}$ & o & $\mathrm{o}$ & $\mathrm{o}$ & o & o \\
\hline $\begin{array}{l}\text { I will try to monitor my use of no-technology } \\
\text { AAC for improvements. }\end{array}$ & o & $\mathrm{O}$ & $\mathrm{o}$ & o & $\mathrm{o}$ \\
\hline I will try to monitor my use of no-technology & & & & & \\
\hline $\begin{array}{l}\text { AAC and make proper adjustments as } \\
\text { needed. }\end{array}$ & o & o & $\mathrm{o}$ & o & o \\
\hline
\end{tabular}

Table B.4: Module 1: Relevance

\begin{tabular}{|c|c|c|c|c|c|}
\hline & $\begin{array}{l}\text { Strongly } \\
\text { disagree }\end{array}$ & $\begin{array}{l}\text { Somewhat } \\
\text { disagree }\end{array}$ & $\begin{array}{l}\text { Neither agree } \\
\text { nor disagree }\end{array}$ & $\begin{array}{l}\text { Somewhat } \\
\text { agree }\end{array}$ & $\begin{array}{l}\text { Strongly } \\
\text { agree }\end{array}$ \\
\hline This topic is relevant to my job. & $\mathrm{O}$ & $\mathrm{O}$ & $\mathrm{O}$ & $\mathrm{O}$ & $\mathrm{O}$ \\
\hline $\begin{array}{l}\text { I will make additional training on this topic } \\
\text { a priority. }\end{array}$ & $\mathrm{o}$ & $\mathrm{O}$ & $\mathrm{O}$ & $\mathrm{O}$ & $\mathrm{O}$ \\
\hline $\begin{array}{l}\text { This is not important for the patients I work } \\
\text { with. }\end{array}$ & $\mathrm{o}$ & $\mathrm{O}$ & $\mathrm{o}$ & $\mathrm{o}$ & $\mathrm{o}$ \\
\hline $\begin{array}{l}\text { I have been looking for training in this } \\
\text { area. }\end{array}$ & $\mathrm{O}$ & $\mathrm{O}$ & $\mathrm{o}$ & $\mathrm{O}$ & $\mathrm{O}$ \\
\hline This was helpful information. & $\mathrm{O}$ & $\mathrm{O}$ & $\mathrm{O}$ & $\mathrm{O}$ & $\mathrm{O}$ \\
\hline I want to learn more on this topic. & $\mathrm{o}$ & $\mathrm{O}$ & o & o & $\mathrm{o}$ \\
\hline I do not know why this training was developed. & $\mathrm{o}$ & $\mathrm{O}$ & $\mathrm{o}$ & $\mathrm{o}$ & $\mathrm{o}$ \\
\hline
\end{tabular}

\section{Additional questions or information you would like.}

Thank you so much for completing MODULE 1 - you have completed 1/3 primary modules (a 4th optional module will be available at the end)! If you would like to take a break now would be a good time to do so, or you may continue on to MODULE 2. 
Table B.5: Module 1: Effectiveness

\begin{tabular}{|c|c|c|c|c|c|}
\hline & $\begin{array}{l}\text { Strongly } \\
\text { disagree }\end{array}$ & $\begin{array}{l}\text { Somewhat } \\
\text { disagree }\end{array}$ & $\begin{array}{l}\text { Neither agree } \\
\text { nor disagree }\end{array}$ & $\begin{array}{l}\text { Somewhat } \\
\text { agree }\end{array}$ & $\begin{array}{l}\text { Strongly } \\
\text { agree }\end{array}$ \\
\hline $\begin{array}{l}\text { The instructor effectively communicated } \\
\text { information. }\end{array}$ & $\mathrm{O}$ & $\mathrm{O}$ & $\mathrm{O}$ & $\mathrm{O}$ & $\mathrm{O}$ \\
\hline Use of time was efficient in this training. & $\mathrm{O}$ & $\mathrm{O}$ & $\mathrm{O}$ & $\mathrm{O}$ & $\mathrm{O}$ \\
\hline This training was too long. & o & $\mathrm{O}$ & $\mathrm{O}$ & o & $\mathrm{O}$ \\
\hline The instructor provided too much information. & $\mathrm{O}$ & $\mathrm{O}$ & $\mathrm{o}$ & o & $\mathrm{o}$ \\
\hline $\begin{array}{l}\text { The instructor spent too much time on basic } \\
\text { information. }\end{array}$ & $\mathrm{O}$ & $\mathrm{O}$ & $\mathrm{O}$ & $\mathrm{O}$ & $\mathrm{O}$ \\
\hline The instructor was engaging. & $\mathrm{O}$ & $\mathrm{O}$ & $\mathrm{O}$ & $\mathrm{O}$ & $\mathrm{O}$ \\
\hline The educational content was engaging. & $\mathrm{o}$ & $\mathrm{o}$ & $\mathrm{o}$ & o & $\mathrm{o}$ \\
\hline I did not understand parts of this training. & $\mathrm{O}$ & $\mathrm{O}$ & $\mathrm{O}$ & $\mathrm{O}$ & $\mathrm{O}$ \\
\hline
\end{tabular}

\section{MODULE 2: Choice-Making and Simple Sign Pre-Test}

1. What does AAC stand for?

2. What are the two parts of communication (choose two)?

o Expressive Communication

o Quiet Communication

o Functional Communication

o Receptive Communication

3. What are two types of AAC that these 3 educational modules focus on (choose two)?
o No-technology AAC
o Low-technology AAC
o Mid-technology AAC
o High-technology AAC

4. What are two ways that you can support choice making for a pediatric patient?

5. What can be adapted on a low-technology communication board?

o Number of pictures/letters

o Size of pictures/letters

o Color

o Organization 
o Number, color, and organization of pictures/letters

o All of the above

6. What are considerations when determining what type of low-technology AAC board to use with a patient (choose all that apply)?

o Literacy

o Vision

o Cognition

o Location

o Language

7. Self-efficacy is defined as a belief in one's capabilities to overcome the demands of a situation in order to achieve a desired outcome. Please move the slider on the scale to indicate your level of self-efficacy regarding your ability to use choice making and low-technology AAC.

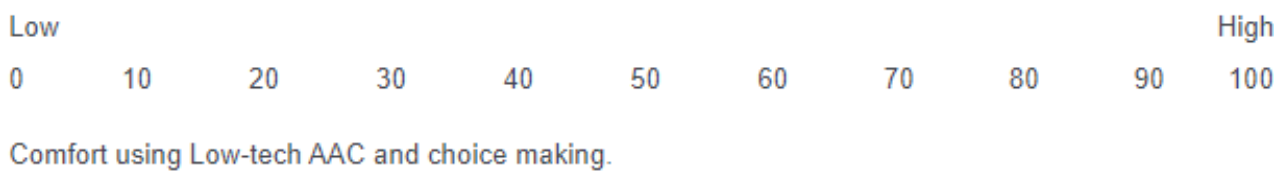

\section{MODULE 2: Choice-Making and Simple Sign Post-Test}

1. What does AAC stand for?

2. What are the two parts of communication (choose two)?

o Expressive Communication

o Quiet Communication

o Functional Communication

o Receptive Communication

3. What are two types of AAC that these 3 educational modules focus on (choose two)?

o No-technology AAC

o Low-technology AAC

o Mid-technology AAC 
o High-technology AAC

4. What are two ways that you can support choice making for a pediatric patient?

5. What can be adapted on a low-technology communication board?

o Number of pictures/letters

o Size of pictures/letters

o Color

o Organization

o Number, color, and organization of pictures/letters

o All of the above

6. What are considerations when determining what type of low-technology AAC board to use with a patient (choose all that apply)?

o Literacy

o Vision

o Cognition

o Location

o Language

7. Self-efficacy is defined as a belief in one's capabilities to overcome the demands of a situation in order to achieve a desired outcome. Please move the slider on the scale to indicate your level of self-efficacy regarding your ability to use choice making and low-technology AAC.

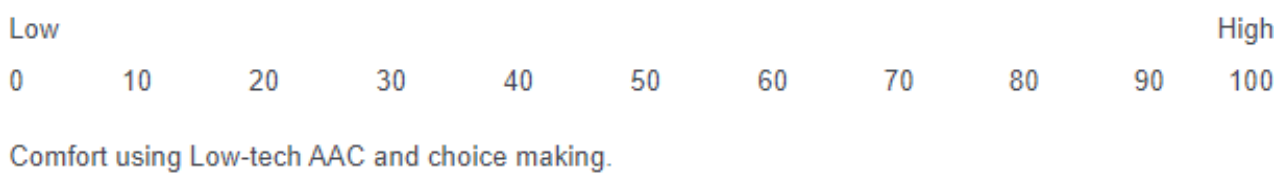

8. What one change will you make in your clinical practice as a result of what you learned in this module?

9. How will your patients benefit from what you have learned?

10. What barriers might make it difficult for you to implement what you have learned (ex. no access to AAC resources)?

11. What facilitators might make it easy to implement what you have learned (ex. support from management to implement communication supports)? 
Table B.6: Module 2: Cognitive L-SES

\begin{tabular}{|c|c|c|c|c|c|}
\hline & $\begin{array}{l}\text { Strongly } \\
\text { disagree }\end{array}$ & $\begin{array}{l}\text { Somewhat } \\
\text { disagree }\end{array}$ & $\begin{array}{l}\text { Neither agree } \\
\text { nor disagree }\end{array}$ & $\begin{array}{l}\text { Somewhat } \\
\text { agree }\end{array}$ & $\begin{array}{l}\text { Strongly } \\
\text { agree }\end{array}$ \\
\hline I can recall how to perform choice making. & $\mathrm{o}$ & $\mathrm{o}$ & $\mathrm{O}$ & $\mathrm{o}$ & $\mathrm{o}$ \\
\hline $\begin{array}{l}\text { I understand the content of choice making } \\
\text { and can demonstrate it to others. }\end{array}$ & $\mathrm{o}$ & $\mathrm{o}$ & $\mathrm{o}$ & $\mathrm{o}$ & $\mathrm{o}$ \\
\hline $\begin{array}{l}\text { I can verbally explain the purpose and } \\
\text { principle of choice making and simple signs. }\end{array}$ & $\mathrm{o}$ & $\mathrm{o}$ & $\mathrm{o}$ & $\mathrm{o}$ & $\mathrm{o}$ \\
\hline $\begin{array}{l}\text { I can verbally explain the sequence and } \\
\text { interrelationship between each step. }\end{array}$ & $\mathrm{o}$ & $\mathrm{o}$ & $\mathrm{o}$ & $\mathrm{o}$ & $\mathrm{o}$ \\
\hline
\end{tabular}

Table B.7: Module 2: Affective L-SES

\begin{tabular}{|c|c|c|c|c|c|}
\hline & $\begin{array}{l}\text { Strongly } \\
\text { disagree }\end{array}$ & $\begin{array}{l}\text { Somewhat } \\
\text { disagree }\end{array}$ & $\begin{array}{l}\text { Neither agree } \\
\text { nor disagree }\end{array}$ & $\begin{array}{l}\text { Somewhat } \\
\text { agree }\end{array}$ & $\begin{array}{l}\text { Strongly } \\
\text { agree }\end{array}$ \\
\hline $\begin{array}{l}\text { I think I spent more time on this module } \\
\text { than on others. }\end{array}$ & $\mathrm{o}$ & $\mathrm{o}$ & $\mathrm{o}$ & $\mathrm{o}$ & $\mathrm{O}$ \\
\hline $\begin{array}{l}\text { I think I gained more from this module } \\
\text { than others. }\end{array}$ & $\mathrm{O}$ & $\mathrm{O}$ & $\mathrm{O}$ & $\mathrm{O}$ & $\mathrm{O}$ \\
\hline $\begin{array}{l}\text { I tend to pay more attention to } \\
\text { information related to this module. }\end{array}$ & $\mathrm{o}$ & $\mathrm{o}$ & $\mathrm{O}$ & $\mathrm{O}$ & $\mathrm{O}$ \\
\hline $\begin{array}{l}\text { I tend to actively look for information } \\
\text { related to this module. }\end{array}$ & $\mathrm{O}$ & $\mathrm{o}$ & $\mathrm{O}$ & $\mathrm{O}$ & $\mathrm{O}$ \\
\hline
\end{tabular}

Table B.8: Module 2: Psychomotor L-SES

\begin{tabular}{|c|c|c|c|c|c|}
\hline & $\begin{array}{l}\text { Strongly } \\
\text { disagree }\end{array}$ & $\begin{array}{l}\text { Somewhat } \\
\text { disagree }\end{array}$ & $\begin{array}{l}\text { Neither agree } \\
\text { nor disagree }\end{array}$ & $\begin{array}{l}\text { Somewhat } \\
\text { agree }\end{array}$ & $\begin{array}{l}\text { Strongly } \\
\text { agree }\end{array}$ \\
\hline $\begin{array}{l}\text { I can precisely imitate the instructor's steps } \\
\text { and actions of choice making. }\end{array}$ & o & $\mathrm{O}$ & $\mathrm{O}$ & $\mathrm{o}$ & o \\
\hline $\begin{array}{l}\text { I can smoothly complete the operational } \\
\text { steps of choice making. }\end{array}$ & $\mathrm{O}$ & $\mathrm{O}$ & $\mathrm{O}$ & $\mathrm{O}$ & $\mathrm{O}$ \\
\hline $\begin{array}{l}\text { I can precisely imitate the instructor's steps } \\
\text { and actions of simple sign. }\end{array}$ & 0 & 0 & $\mathrm{o}$ & $\mathrm{o}$ & 0 \\
\hline $\begin{array}{l}\text { I can smoothly complete the operational } \\
\text { steps of simple signs. }\end{array}$ & o & $\mathrm{O}$ & o & $\mathrm{O}$ & o \\
\hline $\begin{array}{l}\text { I will try to monitor my use of choice making } \\
\text { opportunities for improvements. }\end{array}$ & o & $\mathrm{o}$ & o & $\mathrm{o}$ & $\mathrm{o}$ \\
\hline $\begin{array}{l}\text { I will try to monitor my use of choice making } \\
\text { opportunities and make proper adjustments as } \\
\text { needed. }\end{array}$ & $\mathrm{o}$ & $\mathrm{O}$ & $\mathrm{O}$ & $\mathrm{O}$ & o \\
\hline
\end{tabular}

\section{Additional questions or information you would like.}

Thank you so much for completing MODULE 2 - you have completed 2/3 primary modules (a 4th optional module will be available at the end)! If you would like to take 
Table B.9: Module 2: Relevance

\begin{tabular}{|c|c|c|c|c|c|}
\hline & $\begin{array}{l}\text { Strongly } \\
\text { disagree }\end{array}$ & $\begin{array}{l}\text { Somewhat } \\
\text { disagree }\end{array}$ & $\begin{array}{l}\text { Neither agree } \\
\text { nor disagree }\end{array}$ & $\begin{array}{l}\text { Somewhat } \\
\text { agree }\end{array}$ & $\begin{array}{l}\text { Strongly } \\
\text { agree }\end{array}$ \\
\hline This topic is relevant to my job. & $\mathrm{o}$ & $\mathrm{o}$ & $\mathrm{o}$ & $\mathrm{o}$ & $\mathrm{o}$ \\
\hline $\begin{array}{l}\text { I will make additional training on this topic } \\
\text { a priority. }\end{array}$ & $\mathrm{o}$ & $\mathrm{O}$ & $\mathrm{o}$ & o & o \\
\hline $\begin{array}{l}\text { This is not important for the patients I work } \\
\text { with. }\end{array}$ & o & o & o & $\mathrm{o}$ & $\mathrm{o}$ \\
\hline $\begin{array}{l}\text { I have been looking for training in this } \\
\text { area. }\end{array}$ & $\mathrm{O}$ & $\mathrm{O}$ & o & $\mathrm{o}$ & o \\
\hline This was helpful information. & $\mathrm{o}$ & $\mathrm{o}$ & $\mathrm{o}$ & o & o \\
\hline I want to learn more on this topic. & o & o & o & o & o \\
\hline I do not know why this training was developed. & $\mathrm{o}$ & $\mathrm{o}$ & $\mathrm{o}$ & $\mathrm{o}$ & $\mathrm{o}$ \\
\hline
\end{tabular}

Table B.10: Module 2: Effectiveness

\begin{tabular}{|c|c|c|c|c|c|}
\hline & $\begin{array}{l}\text { Strongly } \\
\text { disagree }\end{array}$ & $\begin{array}{l}\text { Somewhat } \\
\text { disagree }\end{array}$ & $\begin{array}{l}\text { Neither agree } \\
\text { nor disagree }\end{array}$ & $\begin{array}{l}\text { Somewhat } \\
\text { agree }\end{array}$ & $\begin{array}{l}\text { Strongly } \\
\text { agree }\end{array}$ \\
\hline $\begin{array}{l}\text { The instructor effectively communicated } \\
\text { information. }\end{array}$ & $\mathrm{o}$ & $\mathrm{o}$ & $\mathrm{o}$ & $\mathrm{o}$ & $\mathrm{o}$ \\
\hline Use of time was efficient in this training. & $\mathrm{o}$ & $\mathrm{o}$ & $\mathrm{o}$ & o & $\mathrm{o}$ \\
\hline This training was too long. & $\mathrm{o}$ & $\mathrm{o}$ & $\mathrm{o}$ & $\mathrm{o}$ & $\mathrm{o}$ \\
\hline The instructor provided too much information. & o & $\mathrm{o}$ & $\mathrm{o}$ & $\mathrm{o}$ & o \\
\hline $\begin{array}{l}\text { The instructor spent too much time on basic } \\
\text { information. }\end{array}$ & $\mathrm{o}$ & $\mathrm{o}$ & $\mathrm{o}$ & o & $\mathrm{o}$ \\
\hline The instructor was engaging. & o & $\mathrm{o}$ & $\mathrm{o}$ & $\mathrm{o}$ & o \\
\hline The educational content was engaging. & $\mathrm{o}$ & $\mathrm{o}$ & $\mathrm{o}$ & $\mathrm{o}$ & $\mathrm{o}$ \\
\hline I did not understand parts of this training. & o & o & $\mathrm{o}$ & o & o \\
\hline
\end{tabular}

a break now would be a good time to do so, or you may continue on to MODULE 3.

\section{MODULE 3: Partner-Assisted Scanning \\ Pre-Test}

1. What are three examples of no-technology communication strategies (choose three)?

o Wait Time

o Patience

o Recasting

o Providing cues

o Finishing their sentences

o Sign Language 
2. What does AAC stand for?

3. To use partner-assisted scanning, a patient must be able to give a reliable yes/no response.

o True

o False

4. List or describe two patient populations/diagnoses/situations that would benefit from partner-assisted scanning.

5. You ca. use partner-assisted scanning with different kinds of low-tech AAC boards.

o True

o False

6. Self-efficacy is defined as a belief in one's capabilities to overcome the demands of a situation in order to achieve a desired outcome. Please move the slider on the scale to indicate your level of self-efficacy regarding your ability to use partnerassisted scanning and low-technology AAC.

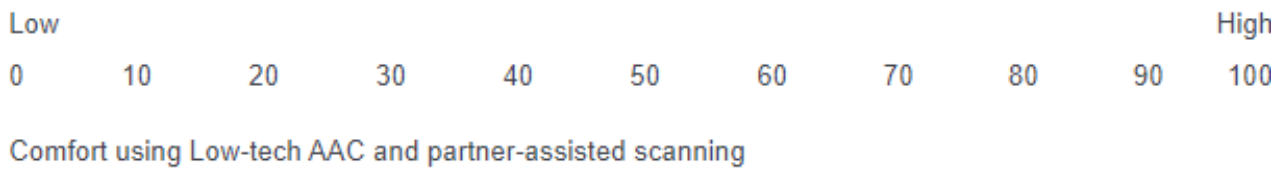

\section{MODULE 3: Partner-Assisted Scanning Post-Test}

1. What are three examples of no-technology communication strategies (choose three)?

o Wait Time

o Patience

o Recasting

o Providing cues

o Finishing their sentences

o Sign Language

2. What does AAC stand for? 
3. To use partner-assisted scanning, a patient must be able to give a reliable yes/no response.

o True

o False

4. List or describe two patient populations/diagnoses/situations that would benefit from partner-assisted scanning.

5. You ca. use partner-assisted scanning with different kinds of low-tech AAC boards.

o True

o False

6. Self-efficacy is defined as a belief in one's capabilities to overcome the demands of a situation in order to achieve a desired outcome. Please move the slider on the scale to indicate your level of self-efficacy regarding your ability to use partnerassisted scanning and low-technology AAC.

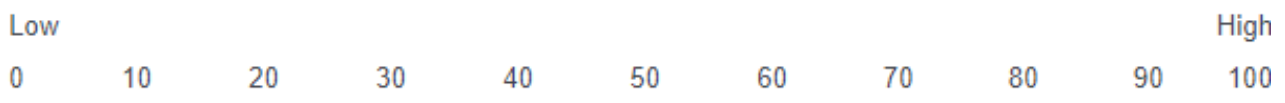

Comfort using Low-tech AAC and partner-assisted scanning

Table B.11: Module 3: Cognitive L-SES

\begin{tabular}{lccccc}
\hline & $\begin{array}{l}\text { Strongly } \\
\text { disagree }\end{array}$ & $\begin{array}{l}\text { Somewhat } \\
\text { disagree }\end{array}$ & $\begin{array}{l}\text { Neither agree } \\
\text { nor disagree }\end{array}$ & $\begin{array}{l}\text { Somewhat } \\
\text { agree }\end{array}$ & $\begin{array}{l}\text { Strongly } \\
\text { agree }\end{array}$ \\
\hline $\begin{array}{l}\text { I can recall how to perform partner-assisted scanning. } \\
\text { I understand the content of partner-assisted scanning } \\
\text { and can demonstrate it to others. }\end{array}$ & $\mathrm{O}$ & $\mathrm{O}$ & $\mathrm{O}$ & $\mathrm{O}$ & $\mathrm{O}$ \\
$\begin{array}{l}\text { I can verbally explain the purpose and } \\
\text { principle of using partner-assisted scanning. }\end{array}$ & $\mathrm{o}$ & $\mathrm{O}$ & $\mathrm{O}$ & $\mathrm{O}$ & $\mathrm{O}$ \\
$\begin{array}{l}\text { I can verbally explain the sequence and } \\
\text { interrelationship between each step. }\end{array}$ & $\mathrm{o}$ & $\mathrm{O}$ & $\mathrm{O}$ & $\mathrm{O}$ & $\mathrm{O}$ \\
\hline
\end{tabular}

7. What one change will you make in your clinical practice as a result of what you learned in this module?

8. How will your patients benefit from what you have learned?

9. What barriers might make it difficult for you to implement what you have learned (ex. no access to AAC resources)?

10. What facilitators might make it easy to implement what you have learned (ex. support from management to implement communication supports)? 
Table B.12: Module 3: Affective L-SES

\begin{tabular}{|c|c|c|c|c|c|}
\hline & $\begin{array}{l}\text { Strongly } \\
\text { disagree }\end{array}$ & $\begin{array}{l}\text { Somewhat } \\
\text { disagree }\end{array}$ & $\begin{array}{l}\text { Neither agree } \\
\text { nor disagree }\end{array}$ & $\begin{array}{l}\text { Somewhat } \\
\text { agree }\end{array}$ & $\begin{array}{l}\text { Strongly } \\
\text { agree }\end{array}$ \\
\hline $\begin{array}{l}\text { I think I spent more time on this module } \\
\text { than on others. }\end{array}$ & $\mathrm{o}$ & $\mathrm{O}$ & $\mathrm{o}$ & o & $\mathrm{o}$ \\
\hline $\begin{array}{l}\text { I think I gained more from this module } \\
\text { than others. }\end{array}$ & $\mathrm{O}$ & $\mathrm{O}$ & $\mathrm{O}$ & $\mathrm{o}$ & $\mathrm{O}$ \\
\hline $\begin{array}{l}\text { I tend to pay more attention to } \\
\text { information related to this module. }\end{array}$ & $\mathrm{O}$ & $\mathrm{O}$ & $\mathrm{O}$ & o & $\mathrm{o}$ \\
\hline $\begin{array}{l}\text { I tend to actively look for information } \\
\text { related to this module. }\end{array}$ & $\mathrm{O}$ & $\mathrm{O}$ & $\mathrm{o}$ & $\mathrm{o}$ & $\mathrm{o}$ \\
\hline
\end{tabular}

Table B.13: Module 3: Psychomotor L-SES

\begin{tabular}{|c|c|c|c|c|c|}
\hline & $\begin{array}{l}\text { Strongly } \\
\text { disagree }\end{array}$ & $\begin{array}{l}\text { Somewhat } \\
\text { disagree }\end{array}$ & $\begin{array}{l}\text { Neither agree } \\
\text { nor disagree }\end{array}$ & $\begin{array}{l}\text { Somewhat } \\
\text { agree }\end{array}$ & $\begin{array}{l}\text { Strongly } \\
\text { agree }\end{array}$ \\
\hline $\begin{array}{l}\text { I can precisely imitate the instructor's steps } \\
\text { and actions of partner-assisted scanning. }\end{array}$ & $\mathrm{O}$ & $\mathrm{O}$ & 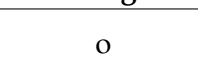 & $\mathrm{O}$ & $\mathrm{O}$ \\
\hline $\begin{array}{l}\text { I can smoothly complete the operational } \\
\text { steps of partner-assisted scanning. }\end{array}$ & o & o & $\mathrm{O}$ & o & $\mathrm{O}$ \\
\hline $\begin{array}{l}\text { I will try to monitor my use of partner-assisted } \\
\text { scanning for improvements. }\end{array}$ & $\mathrm{O}$ & o & o & $\mathrm{O}$ & $\mathrm{o}$ \\
\hline $\begin{array}{l}\text { I will try to monitor my use of partner-assisted } \\
\text { scanning and make proper adjustments as } \\
\text { needed. }\end{array}$ & $\mathrm{O}$ & o & $\mathrm{o}$ & $\mathrm{o}$ & o \\
\hline
\end{tabular}

Table B.14: Module 3: Relevance

\begin{tabular}{|c|c|c|c|c|c|}
\hline & $\begin{array}{l}\text { Strongly } \\
\text { disagree }\end{array}$ & $\begin{array}{l}\text { Somewhat } \\
\text { disagree }\end{array}$ & $\begin{array}{l}\text { Neither agree } \\
\text { nor disagree }\end{array}$ & $\begin{array}{l}\text { Somewhat } \\
\text { agree }\end{array}$ & $\begin{array}{l}\text { Strongly } \\
\text { agree }\end{array}$ \\
\hline This topic is relevant to my job. & $\mathrm{O}$ & o & $\mathrm{o}$ & $\mathrm{o}$ & $\mathrm{o}$ \\
\hline $\begin{array}{l}\text { I will make additional training on this topic } \\
\text { a priority. }\end{array}$ & o & $\mathrm{o}$ & o & o & o \\
\hline $\begin{array}{l}\text { This is not important for the patients I work } \\
\text { with. }\end{array}$ & o & o & o & o & $\mathrm{o}$ \\
\hline $\begin{array}{l}\text { I have been looking for training in this } \\
\text { area. }\end{array}$ & o & $\mathrm{o}$ & o & o & o \\
\hline This was helpful information. & $\mathrm{o}$ & $\mathrm{o}$ & o & $\mathrm{o}$ & o \\
\hline I want to learn more on this topic. & $\mathrm{o}$ & $\mathrm{o}$ & $\mathrm{o}$ & $\mathrm{o}$ & $\mathrm{o}$ \\
\hline I do not know why this training was developed. & $\mathrm{o}$ & $\mathrm{o}$ & $\mathrm{o}$ & $\mathrm{o}$ & $\mathrm{o}$ \\
\hline
\end{tabular}

\section{Additional questions or information you would like.}

\section{Additional Questions about Patients You Work with/will Work with...}


Table B.15: Module 3: Effectiveness

\begin{tabular}{|c|c|c|c|c|c|}
\hline & $\begin{array}{l}\text { Strongly } \\
\text { disagree }\end{array}$ & $\begin{array}{l}\text { Somewhat } \\
\text { disagree }\end{array}$ & $\begin{array}{l}\text { Neither agree } \\
\text { nor disagree }\end{array}$ & $\begin{array}{l}\text { Somewhat } \\
\text { agree }\end{array}$ & $\begin{array}{l}\text { Strongly } \\
\text { agree }\end{array}$ \\
\hline $\begin{array}{l}\text { The instructor effectively communicated } \\
\text { information. }\end{array}$ & $\mathrm{O}$ & $\mathrm{O}$ & $\mathrm{O}$ & $\mathrm{O}$ & $\mathrm{O}$ \\
\hline Use of time was efficient in this training. & $\mathrm{O}$ & $\mathrm{O}$ & $\mathrm{O}$ & $\mathrm{O}$ & $\mathrm{O}$ \\
\hline This training was too long. & $\mathrm{O}$ & $\mathrm{O}$ & $\mathrm{O}$ & $\mathrm{O}$ & $\mathrm{O}$ \\
\hline The instructor provided too much information. & $\mathrm{O}$ & $\mathrm{O}$ & $\mathrm{O}$ & $\mathrm{O}$ & o \\
\hline $\begin{array}{l}\text { The instructor spent too much time on basic } \\
\text { information. }\end{array}$ & $\mathrm{O}$ & $\mathrm{O}$ & $\mathrm{O}$ & $\mathrm{O}$ & $\mathrm{O}$ \\
\hline The instructor was engaging. & $\mathrm{O}$ & $\mathrm{O}$ & $\mathrm{O}$ & $\mathrm{O}$ & $\mathrm{O}$ \\
\hline The educational content was engaging. & $\mathrm{O}$ & $\mathrm{O}$ & $\mathrm{O}$ & $\mathrm{O}$ & $\mathrm{O}$ \\
\hline I did not understand parts of this training. & $\mathrm{O}$ & $\mathrm{O}$ & $\mathrm{O}$ & $\mathrm{o}$ & $\mathrm{O}$ \\
\hline
\end{tabular}

1. What are the top 5 vocabulary words you would include on a low-technology communication board for the patients you work with?

2. How many patients do you think you might use a low-technology AAC board with each week, if they were available to you?
o 0
o $1-2$
o $3-4$
o $5-6$
o $7-8$
o $8+$

3. Do you have access to low-technology AAC boards at your hospital or facility?

o Yes

o No

o I don't know

Skip To: Q98 If Do you have access to low-technology AAC boards at your hospital or facility? $=$ No

4. Could you get access to a low-technology AAC board during your next shift if you needed to?
o Yes
o Maybe
o No 
o I don't know

5. Who provides low-technology AAC boards at your hospital or facility?

o Occupational Therapy

o Speech Language Pathology

o Nursing

o Child Life

o Other

6. How often do you refer to speech therapy for help with communication?

o $<1$ time per month

o 1-2 times per month

o 3-4 times per month

o $>1$ time per week

o Other

7. Would you like additional information on other types of AAC?

o Yes

o No

Thank you so much for completing MODULE 3 - you have completed 3/3 primary modules! If you would like to take a break now would be a good time to do so, or you may continue on to an OPTIONAL MODULE 4 - it will take approximately 15 minutes to complete.

Do you want to watch an OPTIONAL 4th Module on How to Create a Low-Tech AAC Board?

o Yes

o No 


\section{List of References}

All About AAC (2021). Aided I All About AAC (Augmentative E Alternative Communication). URL: https://allaboutaac. wordpress.com/2-2/aided/ (visited on 02/22/2021).

Autism Adventures (2021). Low Tech, Mid Tech and High Tech Assisstive Technology. URL: http://www . autismadventures.com/low-tech-mid-tech-and-high-tech/ (visited on 02/22/2021).

Balandin, S. et al. (2007). "Communicating with nurses: The experiences of 10 adults with cerebral palsy and complex communication needs". In: Applied Nursing Research 20.2, pp. 56-62. ISSN: 08971897. DOI: 10.1016/j . apnr.2006.03.001.

Bandura, A. (1977a). "Self-efficacy: Toward a unifying theory of behavioral change". In: Psychological Review 84.2, pp. 191-215. DOI: 10.1037/0033-295X.84.2.191.

- (1977b). Social learning theory. Englewood Cliffs, NJ: Prentice-Hall, pp. 1-247. ISBN: 9780138167448.

- (1986). Social foundations of thought and action: A social cognitive theory. 1st. Englewood Cliffs, NJ: Prentice-Hall, pp. 1-640. ISBN: 978-0138156145.

- (1998). "Health promotion from the perspective of social cognitive theory". In: Psychology and Health 13.4, pp. 623-649. ISSN: 08870446. DOI: 10.1080/08870449808407422.

Barbu, C. M. (2019). Zoom: A spatial data visualization tool.

Bartlett, G. et al. (2008). "Impact of patient communication problems on the risk of preventable adverse events in acute care settings". In: Canada Medical Association Journal 178.12, pp. 1555-1562. URL: doi : 10.1503/cmaj . 070690.

Beukelman, D. R., L. J. Ball, and S. Fager (2008). "An AAC Personnel Framework : Adults with Acquired Complex Communication Needs". In: Augmentative E Alternative Communication 24.3, pp. 255-267. DOI: 10.1080/07434610802388477.

Billings, D. M. (2000). "A framework for assessing outcomes and practices in Web-based courses in nursing". In: Journal of Nursing Education 39.2, pp. 60-67. ISSN: 00223158. DOI: 10.3928/0148-4834-20000201-07.

Bissinger, Robin and Betty Burns (Oct. 2018). "Third-Party Assessment and Targeted Continuing Education for Maintenance of Certification". In: Journal of Nursing Regulation 9.3, pp. 53-58. ISSN: 21558264. DOI: 10.1016/S2155-8256(18)30153-4.

Blackstone, S. W., D. R. Beukelman, and K. M. Yorkston (2015). Patient-Provider Communication: Roles for Speech-Language Pathologists and Other Health Care Professionals. San Diego, CA: Plural Publishing, pp. 1-339. ISBN: 978-1-59756-574-5. URL: https : //www . pluralpublishing.com/media/media\%7B\%5C_\%7Dppc\%7B\%5C_\%7DSamplePages.pdf. 
Blackstone, S. W. and H. Pressman (Jan. 2016). "Patient communication in health care settings:New opportunities for augmentative and alternative communication". In: Augmentative and Alternative Communication 32.1, pp. 69-79. ISSN: 0743-4618. DOI: 10 .3109/ 07434618 . 2015 . 1125947. URL: http : / / ww . tandfonline . com/doi / full / 10 . 3109 / 07434618.2015 .1125947$.

Blackstone, Sarah W. (2007). "AAC in the ICU”. In: Augmentative Communication News 19.1. Brady, N. C. et al. (2016). "Communication Services and Supports for Individuals with Severe Disabilities: Guidance for Assessment and Intervention". In: American Journal on Intellectual and Developmental Disabilities 121.2, pp. 121-138. ISSN: 0036-8075. DOI: 10.1016/B978-0-12-420118-7.00008-1.Dopamine. arXiv: 15334406.

Braun, V. and V. Clarke (2006). "Using thematic analysis in psychology". In: Qualitative Research in Psychology 3.2, pp. 77-101. ISSN: 14780887. DOI: 10.1191/1478088706qp063oa.

Brunner, Theresa H. et al. (2018). "Feasibility of an iPad to Facilitate Communication in Postoperative Patients With Head and Neck Cancer". In: Journal of Perianesthesia Nursing 33.4, pp. 399-406. ISSN: 10899472. DOI: 10 . 1016/ j . jopan . 2016 .10 . 008. URL: https : //doi.org/10.1016/j.jopan.2016.10.008.

Burkhart, L. J. (2020). ISAAC - What is AAC? URL: https://isaac-online .org/english/ what-is-aac/ (visited on 12/06/2020).

Burns, M. I. et al. (2012). "Training healthcare providers in patient-provider communication: What speech-language pathology and medical education can learn from one another". In: Aphasiology 26.5, pp. 673-688. ISSN: 0268-7038. DOI: 10 .1080/02687038. 2012 .676864.

Buscombe, C. (2013). "Using Gagne's theory to teach procedural skills". In: Clinical Teacher 10.5, pp. 302-307. ISSN: 17434971. DOI: 10.1111/tct.12051.

Campbell, K., V. Taylor, and S. Douglas (2017). “Effectiveness of Online Cancer Education for Nurses and Allied Health Professionals; a Systematic Review Using Kirkpatrick Evaluation Framework". In: Journal of Cancer Education 34.2, pp. 339-356. ISSN: 15430154. DOI: $10.1007 / \mathrm{s} 13187-017-1308-2$.

Chapman, K. B. (2009). "Improving communication among nurses, patients, and physicians: a series of changes leads to cultural transformation at a TCAB hospital." In: American Journal of Nursing 109.11, 21-25 5p. ISSN: 0002-936X. URL: http: // search . ebscohost . com/login . aspx? direct =true $\% 7 \mathrm{~B} \% 5 \mathrm{C} \& \% 7 \mathrm{Ddb}=r z \mathrm{~h} \% 7 \mathrm{~B} \% 5 \mathrm{C} \& \% 7 \mathrm{DAN}=105115506 \% 7 \mathrm{~B} \% 5 \mathrm{C} \&$ $\% 7$ Dsite=ehost-live.

Charmaz, K. (2006). Constructing Grounded Theory: A Practical Guide Through Qualitative Analysis. 1st. Thousand Oaks, CA: Sage Publications Ltd., pp. 1-208. ISBN: 978-0761973539. Cohen, Jacob (1988). Statistical Power Analysis for the Behavioral Sciences. 2nd editio. Lawrence Erlbaum Associates.

Committee on Hospital Care. American Academy of Pediatrics (2012). "Family-centered care and the pediatrician's role." In: Pediatrics 112.3 Pt 1, pp. 394-404. ISSN: 1098-4275. DOI: 10.1542/peds. 2011-3084. URL: http://www.ncbi.nlm.nih.gov/pubmed/12949306.

Communication Community (2021). The AAC files: "mid tech" and "high tech" AAC. URL: https : / / www . communicationcommunity . com / aacfileshightech/ (visited on $02 / 22 / 2021)$. 
Costello, J. M. (2000). "AAC intervention in the intensive care unit: The Children's Hospital Boston model". In: AAC: Augmentative and Alternative Communication 16.3, pp. 137-153. ISSN: 07434618. DOI: 10 . 1080/07434610012331279004.

Costello, J. M., L. Patak, and J. Pritchard (2010). "Communication vulnerable patients in the pediatric ICU: Enhancing care through augmentative and alternative communication". In: Journal of Pediatric Rehabilitation Medicine 3.4, pp. 289-301. ISSN: 18745393. DOI: 10 . 3233/PRM-2010-0140.

Costello, J. M., R. M. Santiago, and S. W. Blackstone (2015). "Pediatric acute and intensive care in hospitals". In: Patient-Provider Communication: Roles for Speech-Language Pathologists and Other Health Care Professionals. Ed. by S. W. Blackstone, D. R. Beukelman, and K. M. Yorkston. San Diego, CA, pp. 187-223.

Coulter, M. A (1990). "A review of two theories of learning and their application in the practice of nurse education". In: Nurse Education Today 10, pp. 333-338.

Dithole, K. et al. (2016). Exploring Communication Challenges Between Nurses and Mechanically Ventilated Patients in the Intensive Care Unit: A Structured Review. DOI: 10.1111/wvn.12146.

Dithole, K. S. et al. (2017). "Communication skills intervention: promoting effective communication between nurses and mechanically ventilated patients." eng. In: BMC Nursing 16, p. 74. ISSN: 1472-6955 (Print). DOI: 10.1186/s12912-017-0268-5.

Divi, C. et al. (2007). "Language proficiency and adverse events in US hospitals: A pilot study". In: International Journal for Quality in Health Care 19.2, pp. 60-67. ISSN: 13534505. DOI: $10.1093 /$ intqhc/mzl069.

Egerod, I. et al. (2020). "Trends and recommendations for critical care nursing research in the Nordic countries: Triangulation of review and survey data". In: Intensive and Critical Care Nursing 56. ISSN: 09643397. DOI: 10.1016/j . iccn.2019.102765.

Ehde, D. M. et al. (2013). “Developing, testing, and sustaining rehabilitation interventions via participatory action research". In: Archives of Physical Medicine and Rehabilitation 94.1 SUPPL. S30-S42. ISSN: 1532821X. DOI: 10 . 1016/ j . apmr . 2012 . 10 . 025. URL: http : //dx.doi.org/10.1016/j.apmr.2012.10.025.

Equility and Human Rights Commission (2014). "Human Rights: Human Lives A Guide to the Human Rights Act for Public Authorities". In: Equality and Human Rights Commission 4.

Fatahi, N. et al. (2010). "Nurse radiographers' experiences of communication with patients who do not speak the native language". In: Journal of Advanced Nursing 66.4, pp. 774-783. ISSN: 03092402. DOI: $10.1111 / \mathrm{j} .1365-2648.2009 .05236 . x$.

Feraco, A. M. et al. (2016). "Communication skills training in pediatric oncology: Moving beyond role modeling". In: Pediatric blood \& cancer 63, pp. 966-972. DOI: 10.1002/pbc25918.

Field, A. (2013). Discovering statistics using IBM SPSS statistics. 4th. Thousand Oaks, CA: Sage Publications Ltd., pp. 1-916.

Films Media Group (2003). Bandura's Social Cognitive Theory: An Introduction. URL: digital. films . com/PortalPlaylists . aspx?wID=98092\%7B\%5C\&\%7Dxtid=44898.

Finke, E. H., J. Light, and L. Kitko (2008). "A systematic review of the effectiveness of nurse communication with patients with complex communication needs with a focus on the 
use of augmentative and alternative communication". In: Journal of Clinical Nursing 17.16, pp. 2102-2115. DOI: $10.1111 / j .1365-2702.2008 .02373 . x$.

Finke, E. H., D. B. Mcnaughton, and K. D. R. Drager (2009). "'All Children Can and Should Have the Opportunity to Learn": General Education Teachers' Perspectives on Including Children with Autism Spectrum Disorder who Require AAC". In: Augmentative $\mathcal{E}$ Alternative Communication 25.2, pp. 110-122. ISSN: 1477-3848. DOI: 10.1080/07434610902886206. URL: http : / / www . tandfonline . com / action / journalinformation? journalCode= iaac20.

Flick, U. (2014). An Introduction to Qualitative Research. 5th ed. London, UK: Sage Publications Ltd.

Gagné, R. M. (1975). Essentials of learning for instruction. Hinsdale, Illinois: The Dryden Press, pp. 1-184. ISBN: 978-0132862530.

- (1984). "Learning Outcomes and Their Effects". In: American Psychologist 39.4, pp. 377-385.

- (1985). The Conditions of Learning. 4th. New York, NY: Holt, Rinehart, and Winston. ISBN: 978-0030636882.

Gerkin, K. L., T. H. Taylor, and F. M. Weatherby (2009). “The perception of learning and satisfaction of nurses in the online environment". In: Journal for Nurses in Staff Development 25.1, E8-E13. ISSN: 15389049. DOI: 10.1097/NND . 0b013e318194b6a4.

Glaser, B. G. and A. L. Strauss (1967). "The discovery of grounded theory". In: International Journal of Qualitative Methods 5, pp. 1-10. ISSN: 16094069. DOI: 10 . 2307 / 588533. URL: http://www . ualberta.ca/\%7B \%7Diiqm/backissues/5\%7B\%5C_\%7D1/pdf/mills.pdf.

GovTrack.us (2019). S. 2561 - 100th Congress: Technology-related assistance for individuals with disabilities act of 1988. Tech. rep. URL: https://www . govtrack. us/congress/bills/100/ s2561.

Happ, M. B., B. M. Baumann, et al. (2010). "SPEACS-2: Intensive care unit "communication rounds" with speech language pathology". In: Geriatric Nursing 31.3, pp. 170-177. ISSN: 01974572. DOI: 10.1016/j.gerinurse.2010.03.004. URL: http://dx.doi.org/10.1016/ j.gerinurse.2010.03.004.

Happ, M. B., K. L. Garrett, et al. (2014). "Effect of a multi-level intervention on nurse-patient communication in the intensive care unit: Results of the SPEACS trial". In: Heart and Lung: Journal of Acute and Critical Care 43.2, pp. 89-98. ISSN: 01479563. DOI: 10.1016/j . hrtlng. 2013.11.010. URL: http://dx.doi.org/10.1016/j.hrtlng. 2013.11.010.

Happ, M. B., S. Sereika, et al. (2008). "Use of the quasi-experimental sequential cohort design in the Study of Patient-Nurse Effectiveness with Assisted Communication Strategies (SPEACS)". In: Contemporary Clinical Trials 29.5, pp. 801-808. ISSN: 15517144. DOI: 10 . 1016/j.cct.2008.05.010. URL: http://dx.doi.org/10.1016/j.cct.2008.05.010.

Hemsley, B. and S. Balandin (2007). "A metasynthesis of patient-provider communication in hospital for patients with severe communication disabilities: Informing new translational research". In: AAC: Augmentative and Alternative Communication 30.4, pp. 329-343. DOI: 10.3109/07434618.2014.955614.

- (2014). "A metasynthesis of patient-provider communication in hospital for patients with severe communication disabilities: Informing new translational research". In: $A A C$ : 
Augmentative and Alternative Communication 30.4, pp. 329-343. DOI: 10.3109/07434618. 2014.955614.

Hemsley, B., S. Balandin, and L. Togher (2008a). "Family caregivers discuss roles and needs in supporting adults with cerebral palsy and complex communication needs in the hospital setting". In: Journal of Developmental and Physical Disabilities 20.3, pp. 257-274. ISSN: 1056263X. DOI: 10.1007/s10882-007-9095-z.

- (2008b). "Professionals' views on the roles and needs of family carers of adults with cerebral palsy and complex communication needs in hospital". In: Journal of Intellectual and Developmental Disability 33.2, pp. 127-136. ISSN: 13668250. DOI: 10 . 1080 / 13668250802082898.

Hemsley, B., S. Balandin, and L. Worrall (2011). "Nursing the patient with developmental disability in hospital: Roles of paid carers". In: Qualitative Health Research 21.12, pp. 16321642. ISSN: 10497323. DOI: 10.1177/1049732311415289.

Hemsley, B., S. Lee, et al. (2013). "Supporting communication for children with cerebral palsy in hospital: Views of community and hospital staff." In: Developmental neurorehabilitation 17.June, pp. 156-166. ISSN: 1751-8431. DOI: 10.3109/17518423.2012.741149. URL: http: //www.ncbi.nlm.nih.gov/pubmed/24102353.

Holden, K. (2017). “No longer voiceless in the ICU”. In: ASHA Leader 22.12, pp. 40-41.

Hopf, S. C. (2018). "Communication Capacity Research in the Majority World: Supporting the human right to communication specialist services". In: International Journal of Speech-Language Pathology 20.1, pp. 84-88. ISSN: 17549507. DOI: 10.1080/17549507.2018. 1400101. URL: https://doi.org/10.1080/17549507.2018.1400101.

Hosseini, S. R., M. A. Valizad-Hasanloei, and A. Feizi (2018). "The effect of using communication boards on ease of communication and anxiety in mechanically ventilated conscious patients admitted to intensive care units". In: Iranian Journal of Nursing and Midwifery Research 23.5, pp. 358-362. ISSN: 22285504. DOI: 10.4103/ijnmr. IJNMR_68_17.

Hurtig, R. R. et al. (2019). "Improving Patient Safety and Patient-Provider Communication". In: Perspectives of the ASHA Special Interest Groups 4.5, pp. 1017-1027. ISSN: 2381-4764. DOI: 10.1044/2019_pers-sig12-2019-0021.

IBM Corp. (2017). IBM SPSS Statistics for Windows. Armonk, NY.

Johnson, E., S. Nilsson, and M. Adolfsson (2015). “Eina ! Ouch ! Eish ! Professionals ' Perceptions of How Children with Cerebral Palsy Communicate About Pain in South African School Settings : Implications for the use of AAC". In: Augmentative and Alternative Communication October, pp. 1-11. ISSN: 0743-4618. DOI: 10.3109/07434618.2015.1084042.

Kang, Y. N. et al. (2019). "Development of a short and universal learning self-efficacy scale for clinical skills". In: PLoS ONE 14.1, pp. 1-11. ISSN: 19326203. DOI: 10.1371/ journal. pone. 0209155.

Karlsen, M. M. W., M. A. Ølnes, and L. G. Heyn (2019). “Communication with patients in intensive care units: a scoping review". In: Nursing in Critical Care 24.3, pp. 115-131. ISSN: 14785153. DOI: $10.1111 /$ nicc. 12377.

Kemper, K. J. et al. (2006). "Randomized controlled trial comparing four strategies for delivering e-curriculum to health care professionals [ISRCTN88148532]". In: BMC Medical Education 6, pp. 1-11. ISSN: 14726920. DOI: 10.1186/1472-6920-6-2. 
Keough, V. A. and P. Tanabe (2011). "Survey Research: An Effective Design for Conducting Nursing Research". In: Journal of Nursing Regulation 1.4, pp. 37-44. ISSN: 21558264. DOI: 10 . 1016 / S2155 - 8256 (15 ) 30315 - X. URL: http : / / dx . doi . org / 10 . 1016 / S2155 8256 (15) 30315-X.

Khadjooi, K., K. Rostami, and S. Ishaq (2011). "How to use Gagne's model of instructional design in teaching psychomotor skills". In: Gastroenterology and Hepatology From Bed to Bench 4.3, pp. 116-119. URL: https : / / pdfs . semanticscholar . org / 582a / 124a7caa64456626aa462482e8d3cdeacca8.pdf.

Koszalinski, R. S., R. M. Tappen, and D. Viggiano (2015). "Evaluation of Speak for Myself with Patients Who Are Voiceless." In: Rehabilitation nursing : the official journal of the Association of Rehabilitation Nurses 40.4, p. 235. ISSN: 0278-4807. DOI: http: //dx . doi . org/ 10.1002/rnj. 186.

Kruijver, I. P. M. et al. (2000). "Evaluation of communication training programs in nursing care: A review of the literature". In: Patient Education and Counseling 39.1, pp. 129-145. ISSN: 07383991. DOI: 10.1016/S0738-3991(99)00096-8.

Lahti, M. et al. (2014). "Knowledge transfer from an e-learning course to clinical practice". In: Nurse Education Today 34.5, pp. 842-847. ISSN: 15322793. DOI: 10.1016/j . nedt. 2013. 09.003. URL: http://dx.doi.org/10.1016/j.nedt.2013.09.003.

Lewis, M. J. et al. (Jan. 2001). "A review of evaluative studies of computer-based learning in nursing education". In: Nurse Education Today 21.1, pp. 26-37. ISSN: 02606917. DOI: 10.1054 /nedt. 2000.0494.

Light, J. and K. Drager (2007). "AAC technologies for young children with complex communication needs: state of the science and future research directions." In: Augmentative and Alternative Communication 23.3, pp. 204-16. ISSN: 1477-3848. DOI: 10 . 1080 / 07434610701553635. URL: http://www.ncbi.nlm.nih.gov/pubmed/17701740.

Light, J. and D. McNaughton (2012). "Supporting the Communication, Language, and Literacy Development of Children with Complex Communication Needs: State of the Science and Future Research Priorities". In: Assistive Technology 24.1, pp. 34-44. ISSN: 1040-0435. DOI: 10.1080/10400435.2011.648717.

Light, J. and D. Mcnaughton (2015). “Designing AAC research and intervention to improve outcomes for individuals with complex communication needs". In: Augmentative $\mathcal{E}$ Alternative Communication 31.2, pp. 85-96. ISSN: 1477-3848. DOI: 10 . 3109/07434618. 2015 . 1036458. URL: http : / / www . tandf online . com/action/ journalinformation? journalCode=iaac20.

Light, J., D. McNaughton, et al. (2019). “Challenges and opportunities in augmentative and alternative communication: Research and technology development to enhance communication and participation for individuals with complex communication needs". In: Augmentative E Alternative Communication 35.1, pp. 1-12. ISSN: 07434618. DOI: 10.1080/ 07434618.2018 .1556732$.

Light, J., R. Page, et al. (2007). "Children's ideas for the design of AAC assistive technologies for young children with complex communication needs." In: Augmentative and Alternative Communication 23.4, pp. 274-87. ISSN: 1477-3848. DOI: 10.1080/07434610701390475. URL: http://www.ncbi.nlm.nih.gov/pubmed/17852057. 
Markov, M. and A. Hazan (2012). "Advances in communication technology: Implications for new nursing skills". In: Journal of Pediatric Nursing 27.5, pp. 591-593. ISSN: 08825963. DOI: 10.1016/j . pedn. 2012.07.004. URL: http://dx . doi .org/10 .1016/j . pedn. 2012 . 07.004.

Marx, A. and M. Locast (n.d.). AAC Glossary of Terms. Tech. rep. University of Wisconsin Hospital and Clinics, pp. 1-5. URL: https : / www . aacpdm . org/UserFiles/file/IC2Marx-2.pdf.

McCarthy, B., M. O’Donovan, and A. Twomey (2008). “Person-centred communication: Design, implementation and evaluation of a communication skills module for undergraduate nursing students - An Irish context". In: Contemporary Nurse 27.2, pp. 207-222. ISSN: 10376178. DOI: 10.5172/conu .2008.27.2.207.

McLeod, S. (2018). "Communication rights: Fundamental human rights for all". In: International Journal of Speech-Language Pathology 20.1, pp. 3-11. ISSN: 17549507. DOI: 10 . $1080 /$ 17549507.2018.1428687. URL: https://doi.org/10.1080/17549507.2018.1428687.

Mesko, P. J. et al. (Dec. 2011). "Use of picture communication aids to assess pain location in pediatric postoperative patients." English. In: Journal of perianesthesia nursing : official journal of the American Society of PeriAnesthesia Nurses 26.6, pp. 395-404. ISSN: 1532-8473 (Electronic). DOI: 10.1016/j.jopan.2011.09.006.

Miner, A. et al. (2015). “Using Gagne's 9 events of instruction to enhance student performance and course evaluations in undergraduate nursing course". In: HHS Public Access 40.3, pp. 152-154. ISSN: 1527-5418. DOI: 110.1016/j . bbi.2017.04.008. arXiv: 15334406.

Moorcroft, A., N. Scarinci, and C. Meyer (2019). "A systematic review of the barriers and facilitators to the provision and use of low-tech and unaided AAC systems for people with complex communication needs and their families". In: Disability and Rehabilitation: Assistive Technology 14.7, pp. 710-731. ISSN: 17483115. DOI: 10 . 1080/17483107 . 2018 . 1499135. URL: https://doi.org/10.1080/17483107.2018.1499135.

Morris, Megan A., Brian J. Dudgeon, and Kathryn Yorkston (2013). "A qualitative study of adult AAC users' experiences communicating with medical providers". In: Disability and Rehabilitation: Assistive Technology 8.6, pp. 472-481. ISSN: 17483107. DOI: 10 . 3109 / 17483107.2012 .746398$.

National Commission on Correctional Health Care (2011). Therapeutic Communication and Behavioral Management. URL: https://www .ncchc.org/cnp-therapeutic-communication (visited on 03/04/2021).

Nguyen, T. (2015). "The Effectiveness of Online Learning: Beyond No Significant Difference and Future Horizons". In: MERLOT Journal of Online Learning and Teaching 11.2, pp. 309319.

Nicoll, P. et al. (2018). "Evaluation of technology-enhanced learning programs for health care professionals: Systematic review". In: Journal of Medical Internet Research 20.4, pp. 1-9. ISSN: 14388871. DOI: 10.2196/jmir. 9085.

Noguchi, Ayako, Tomoko Inoue, and Isao Yokota (2019). "Promoting a nursing team's ability to notice intent to communicate in lightly sedated mechanically ventilated patients in an intensive care unit: An action research study". In: Intensive and Critical Care Nursing 51, pp. 64-72. ISSN: 09643397. DOI: 10.1016/j . iccn.2018.10.006. 
O'Fallon, L. R. and A. Dearry (2002). "Community-based participatory research as a tool to advance environmental health sciences". In: Environmental Health Perspectives 110.SUPPL. 2, pp. 155-159. ISSN: 00916765. DOI: 10.1289/ehp.02110s2155.

Ong, L. M.L. et al. (1995). "Doctor-patient communication: A review of the literature". In: Social Science and Medicine 40.7, pp. 903-918. ISSN: 02779536. DOI: 10 . 1016/02779536 (94) 00155-M.

Otuzoğlu, Münevver and Azize Karahan (2014). “Determining the effectiveness of illustrated communication material for communication with intubated patients at an intensive care unit". In: International Journal of Nursing Practice 20.5, pp. 490-498. ISSN: 1440172X. DOI: $10.1111 /$ ijn. 12190.

Pangh, B. et al. (2019). "The Effect of Reflection on Nurse-Patient Communication Skills in Emergency Medical Centers". In: Journal of Caring Sciences 8.2, pp. 75-81. ISSN: 2251-9920. DOI: $10.15171 /$ jcs.2019.011.

Patak, L. et al. (2009). "Improving patient-provider communication: a call to action." In: The Journal of nursing administration 39.9, pp. 372-6. ISSN: 1539-0721. DOI: 10.1097/NNA . Ob013e3181b414ca. URL: http: / / www . ncbi . nlm. nih. gov/pubmed / 19745632\%7B\%5C\% \%7D0Ahttp: //www. pubmedcentral.nih.gov/articlerender.fcgi?artid=PMC2904301.

Patak, Lance et al. (2006). "Communication boards in critical care: patients' views". In: Applied Nursing Research 19.4, pp. 182-190. ISSN: 08971897. DOI: 10.1016/j . apnr . 2005. 09.006.

QSR International Pty Ltd. (2019). NVivo qualitative data analysis software.

Radtke, J. V., B. M. Baumann, et al. (2011). "Listening to the Voiceless Patient: Case Reports in Assisted Communication in the Intensive Care Unit". In: Journal of Palliative Medicine 14.6, pp. 791-795. ISSN: 1096-6218. DOI: 10 . 1089/ jpm . 2010 . 0313. URL: http: / / www . liebertpub.com/doi/10.1089/jpm.2010.0313.

Radtke, J. V., J. A. Tate, and M. B. Happ (2012). “Nurses' perceptions of communication training in the ICU". In: Intensive and Critical Care Nursing 28.1, pp. 16-25. ISSN: 09643397. DOI: 10.1016/j . iccn . 2011 .11 .005. arXiv: NIHMS150003. URL: http://dx . doi .org/10 . $1016 / j$.iccn.2011.11.005.

Rao, J. K. et al. (2007). "Communication interventions make a difference in conversations between physicians and patients: A systematic review of the evidence". In: Medical Care 45.4, pp. 340-349. ISSN: 00257079. DOI: 10.1097/01 . mlr.0000254516.04961.d5.

Rao, P. R. (Nov. 2011). “Our Role in Effective Patient-Provider Communication". In: The ASHA Leader 16.13, pp. 17-18. ISSN: 1085-9586. DOI: 10.1044/leader . FTP .16132011.17. URL: http://pubs . asha.org/doi/10.1044/leader.FTP.16132011.17.

Rimal, R. N. and M. K. Lapinski (Apr. 2009). "Why health communication is important in public health". In: Bulletin of the World Health Organization 87.4, p. 247. ISSN: 00429686. DOI: 10.2471/BLT .08.056713. URL: http: //www . who. int/bulletin/volumes/87/4/08056713.pdf.

Rodriguez, C. S., H. J. Spring, and M. Rowe (2018). “Effectiveness of a technology-based communication intervention for suddenly speechless patients in critical care units: Nurses perceptions and experiences". In: Cogent Social Sciences 4.1, pp. 1-14. ISSN: 23311886. DOI: 10.1080/23311886.2018.1552734. 
Rogers, C. (1983). Freedom to Learn for the 80s. Columbus, OH: Charles E. Merrill Publishing Company.

Schinke, P. et al. (1981). "Measuring the Impact of Continuing Education". In: Journal of Education for Social Work 17.1, pp. 59-64.

Schwartz, I. S. and D. M. Baer (1991). "Social Validity Assessments: Is Current Practice State of the Art?" In: Journal of Applied Behavior Analysis 24.2, pp. 189-204. DOI: 10.1901/jaba. 1991.24-189.

Shorey, S. et al. (2018). "Blended learning pedagogy designed for communication module among undergraduate nursing students: A quasi-experimental study". In: Nurse Education Today 61.November 2017, pp. 120-126. ISSN: 15322793. DOI: 10 .1016/j . nedt . 2017 . 11. 011. URL: https://doi.org/10.1016/j.nedt.2017.11.011.

Simmons, A. et al. (2019). "Knowledge and experiences with augmentative and alternative communication by paediatric nurses: A pilot study". In: Disability and Rehabilitation: Assistive Technology, pp. 1-13. ISSN: 17483115. DOI: 10.1080/17483107.2019.1685015.

El-Soussi, Azza H. et al. (2015). “Augmented alternative communication methods in intubated COPD patients: Does it make difference". In: Egyptian Journal of Chest Diseases and Tuberculosis 64.1, pp. 21-28. ISSN: 04227638. DOI: 10.1016/j . ejcdt . 2014.07.006. URL: http://dx.doi.org/10.1016/j.ejcdt.2014.07.006.

Stewart, M. A. (1995). "Effective physician-patient communication and health outcomes: A review". In: Canadian Medical Association Journal 152.9, pp. 1423-1433. ISSN: 08203946.

The Joint Commission (2010). Advancing effective communication, cultural competence, and patient-and family-centered care: A roadmap for hospitals. Tech. rep. Oakbrook Terrace, IL: The Joint Commission, pp. 1-94.

- (2011). R3 Report Requirement, Rationale, Reference. Tech. rep., pp. 1-4. URL: www . joint commission. org.

- (2020). Sentinel Event. URL: https : //www . jointcommission . org/resources/patientsafety-topics/sentinel-event/ (visited on 08/13/2020).

Therrien, M. C and J. Light (2018). "Promoting Peer Interaction for Preschool Children With Complex Communication Needs and Autism Spectrum Disorder." In: American Journal of Speech-Language Pathology 27.1, pp. 207-221. ISSN: 10580360. URL: http: //10 . 0 4 . 20/ 2017\%7B\%5C_\%7DAJSLP-17-0104\%7B\%5C\%\%7D0Ahttps ://search. ebscohost. com/login . aspx?direct=true $\% 7 \mathrm{~B} \% 5 \mathrm{C} \& \% 7 \mathrm{Ddb}=$ eue $\% 7 \mathrm{~B} \% 5 \mathrm{C} \& \% 7 \mathrm{DAN}=127887182 \% 7 \mathrm{~B} \% 5 \mathrm{C} \& \% 7 \mathrm{D}$ site=ehostlive.

Thunberg, G., M. Buchholz, and S. Nilsson (2016). "Strategies that assist children with communicative disability during hospital stay : Parents ' perceptions and ideas". In: Journal of Child Health Care 20.2, pp. 224-233. DOI: 10.1177/1367493514568298.

Thunberg, G., C. J. Törnhage, and S. Nilsson (2016). "Evaluating the Impact of AAC Interventions in Reducing Hospitalization-related Stress: Challenges and Possibilities". In: AAC: Augmentative and Alternative Communication 32.2, pp. 143-150. ISSN: 14773848. DOI: 10.3109/07434618.2016.1157703.

Turnbull, H. R. and A. P. Turnbull (1991). Participatory Action Research and Public Policy. Tech. rep. Lawrence, KS: Beach Center on Families and Disabilities, pp. 3-25. 
UN Commission on Human Rights (1990). Convention on the Rights of the Child. Tech. rep. URL: https://www.refworld.org/docid/3b00f03d30.html.

United Nations (1948). “United Nations Human Rights Declaration”. In: ISSN: 19410832. URL: https : //www . ohchr.org/EN/UDHR/Documents/UDHR\%7B\%5C_\%7DTranslations/eng.pdf.

USSAAC (2021). AAC Devices. URL: https : //ussaac . org/aac-info/aac-devices/ (visited on $02 / 22 / 2021$ ).

White, G. W., M. Suchowierska, and M. Campbell (2004). "Developing and systematically implementing participatory action research". In: Archives of Physical Medicine and Rehabilitation 85.SUPPL. 2, pp. 3-12. ISSN: 00039993. DOI: 10.1016/j . apmr . 2003.08.109.

Wojnicki-Johansson, G. (2001). "Communication between nurse and patient during ventilator treatment: Patient reports and RN evaluations". In: Intensive and Critical Care Nursing 17.1, pp. 29-39. ISSN: 09643397. DOI: 10.1054/iccn.2000.1547.

Wolf, D. M. et al. (2008). "Effect of patient-centered care on patient satisfaction and quality of care". In: Journal of Nursing Care Quality 23.4, pp. 316-321. ISSN: 10573631. DOI: 10 . 1097/01. NCQ.0000336672.02725.a5.

Wolf, M. M. (1978). "Social validity: The case for subjective measurement or how applied behavior analysis is finding its heart". In: Journal of Applied Behavior Analysis 11.2, pp. 203214. ISSN: 00218855. DOI: 10.1901/jaba.1978.11-203.

Wong, Y. L. (2018). "Utilizing the principles of gagne's nine events of instruction in the teaching of goldmann applanation tonometry". In: Advances in Medical Education and Practice 9, pp. 45-51. ISSN: 11797258. DOI: 10.2147/AMEP.S145498.

World Health Organisation (2011). "WHO Disability Report". In: World Report on Disability, pp. 1-325. URL: http://www. who.int/disabilities/world\%7B\%5C_\%7Dreport/2011/ report.pdf.

Zabar, S. et al. (2006). "'Oh! She doesn't speak english!" Assessing resident competence in managing: Linguistic and cultural barriers". In: Journal of General Internal Medicine 21.5, pp. 510-513. ISSN: 08848734. DOI: 10.1111/j.1525-1497.2006.00439.x.

Zaga, C. J., S. Berney, and A. P. Vogel (2019). “The feasibility, utility, and safety of communication interventions with mechanically ventilated intensive care unit patients: A systematic review". In: American Journal of Speech-Language Pathology 28.3, pp. 1335-1355. ISSN: 15589110. DOI: 10.1044/2019_AJSLP-19-0001.

Ziegler, S. M. et al. (2005). "Bandura's Social Cognitive Theory". In: Theory-Directed Nursing Practice. Ed. by S. M. Ziegler. 2nd. New York, NY: Springer Publishing Company, Inc. Chap. 3, pp. 29-55. 


\section{Vita}

Amanda K. Simmons was born in Rocky Mount, NC in 1987. She studied and earned a Bachelor of Music - Vocal Performance at the University of North Carolina - Chapel Hill in 2009. She went on to spend two and a half years as a Peace Corps Volunteer in West and Southern Africa. She completed her initial 27 months of Peace Corps service as a community health education volunteer in Cameroon in 2011 and followed with a short stint of service in Zambia as a maternal child health specialist with Peace Corps Response in 2012.

Amanda began her graduate career, pursing a Masters in Speech Language Pathology at the University of Tennessee Health Science Center (UTHSC), conferring this degree in 2015. She began her doctoral work in 2015 under the direction of Dr. Jillian McCarthy at UTHSC. She simultaneously completed her clinical fellowship year and obtained her Certificate of Clinical Competence in 2016. Simmons expects to complete her doctoral degree in Spring 2021 and plans to continue her academic career through teaching, clinical work and clinically-based research pursuits. 